\title{
Auslander-Reiten Triangles, Ziegler Spectra and Gorenstein Rings
}

Dedicated to Prof. Idun Reiten on her 62nd birthday

\author{
APOSTOLOS BELIGIANNIS \\ Department of Mathematics, University of the Aegean, 83200 Karlovassi, Samos, Greece. \\ e-mail: abeligia@aegean.gr
}

(Received: June 2003)

\begin{abstract}
We investigate (existence of) Auslander-Reiten triangles in a triangulated category in connection with torsion pairs, existence of Serre functors, representability of homological functors and realizability of injective modules. We also develop an Auslander-Reiten theory in a compactly generated triangulated category and we study the connections with the naturally associated Ziegler spectrum. Our analysis is based on the relative homological theory of purity and Brown's Representability Theorem. Our main interest lies in the structure of Auslander-Reiten triangles in the full subcategory of compact objects. We also study the connections and the interplay between Auslander-Reiten theory, pure-semisimplicity and the finite type property, Grothendieck groups, and we give applications to derived categories of Gorenstein rings.
\end{abstract}

Mathematical Subject Classification (2000): Primary 18E30, 16G70, 18E40, 55U35, 18G55, 18G60; Secondary 16G60, 18E10, 18F30.

Keywords: Auslander-Reiten Triangles, Compact and Pure-Injective Objects, Gorenstein Rings, Grothendieck Groups, Purity, Serre Functors, Triangulated and Derived Categories, Ziegler Spectrum.

\section{Introduction}

Auslander and Reiten introduced in the early seventies almost split sequences, now called Auslander-Reiten sequences, in the representation theory of Artin algebras [5]. Since then Auslander-Reiten theory together with its companion theory of purity of modules became an indispensable tool for the structural analysis of a module category. On the other hand there is a close relationship between purity and model theory of modules culminating in the study of pure-injectivity in connection with the Ziegler spectrum of a module category introduced by Ziegler [80] in model theoretic terms. This fruitful relationship and interplay produced many important results in both directions as documented in the book of Prest [68]. In this connection Prest in the mid-eighties was the first who indicated a close relationship between Auslander-Reiten theory and the structure of the Ziegler spectrum of a module category, see [68]. Notice that Auslander-Reiten theory mainly concerns the behavior of finitely generated modules whereas the Ziegler spectrum controls the complexity of the whole module category. We refer to the work of Prest [68], Herzog [41] and Krause [53] for comprehensive treatments of the subject. 
Generalizing part of these developments Happel in the mid-eighties [36] introduced triangulated categories in representation theory offering in this way new invariants and classification limits in the study of representations. One of the most successful developments in this setting is Happel's extension of Auslander-Reiten theory in the bounded derived category of an Artin algebra. Since triangulated categories provide the natural setting for the investigation of several homological or representation theoretic problems in representation theory, algebraic geometry and algebraic topology (see for instance Serre and Poincare duality [23,44,75]), the above developments provide strong motivation for the investigation of Auslander-Reiten theory in more general triangulated categories, for instance those which may be of interest in non-commutative geometry, in connection with suitably defined notions of purity and the Ziegler spectrum.

On the other hand most of the triangulated categories which occur in practice are compactly generated in the sense of Neeman [64], for instance the stable homotopy category of spectra, the derived category of quasi-coherent sheaves over a quasi-compact separated scheme, the derived category of modules over a ring, and the stable module category of a modular group algebra or more generally a quasi-Frobenius ring. In this framework a concept of purity has been introduced by Krause [51] and the author [18], and the first direct connections between purity, the Ziegler spectrum and Auslander-Reiten theory were developed in $[19,52]$.

Our main aim in this paper is to make a detailed systematic investigation of Auslander-Reiten theory in, mainly compactly generated, triangulated categories and to study the interplay and the connections with the theory of purity and the Ziegler spectrum in the triangulated level. In analogy with Auslander-Reiten theory in module categories, we study the connections with Grothendieck groups, torsion pairs, representation embeddings, and pure-semisimplicity or the finite type property. Since Auslander-Reiten theory imposes several finiteness conditions, in the working setting of a compactly generated triangulated category $\mathcal{C}$, it behaves better when we restrict our attention to suitable "finite" objects. Therefore we are mainly interested in constructing a satisfactory Auslander-Reiten theory in the full subcategory of compact objects. In turn this theory gives valuable information for the Ziegler spectrum which controls the behavior of $\mathcal{C}$ in a certain sense.

Existence of Auslander-Reiten sequences or triangles is, of course, of central importance in both the abelian and triangulated setting and is related to the question of representability of functors and/or realizability of injective modules, so it is crucial to have representability theorems, like Brown's representability, at our disposal. Working in suitable categories of finite objects, global existence of Auslander-Reiten triangles gives a pleasant behavior to the category and is related to existence of Serre functors and a 
certain non-singularity or Gorensteinness property expressed by some form of duality.

The paper is divided roughly in three parts. We devote the first part, consisting of Sections $2-5$, to the study of global existence of Auslander-Reiten triangles in various settings and we give the connections with Serre functors, dualizing categories and torsion pairs. In the second part, consisting of Sections 6-9, we concentrate on Auslander-Reiten theory in a compactly generated category, we give the connections with purity, the Ziegler spectrum and derived categories and we present methods for constructing categories with Auslander-Reiten triangles. The last part, consisting of Sections 10-12, is devoted to the study of Auslander-Reiten theory in connection with pure-semisimplicity and the finite type property, endofiniteness and Grothendieck groups. We refer to the text for the precise statements of our results, noting that many of them are triangulated analogues and extensions of well-known ring and module theoretic results proved by different methods, so in this way the range of their applications is widened.

CONVENTION. The composition of morphisms in a given category is meant in the diagrammatic order: the composition of $f: A \rightarrow B$ with $g: B \rightarrow C$ is denoted by $f \circ g: A \rightarrow C$. Our additive categories admit finite direct sums.

\section{Realizability of Injectives and Construction of Morphisms}

In this section we present a method for constructing morphisms with prescribed properties in an additive category. The method, which will be important later in connection with existence of Auslander-Reiten triangles, is based on representability of injective envelopes of simple functors and realizability of injective modules.

Throughout we fix an additive category $\mathcal{C}$ with split idempotents. If $\Lambda$ is a ring then we denote by Mod- $\Lambda$ the category of right $\Lambda$-modules. Left $\Lambda$-modules are treated as right $\Lambda^{\mathrm{op}}$-modules, where $\Lambda^{\mathrm{op}}$ is the opposite ring of $\Lambda$.

\subsection{INJECTIVE ENVELOPES OF SIMPLE MODULES AND FUNCTORS}

We fix an object $T$ in $\mathcal{C}$ and we denote by $\Lambda_{T}:=\operatorname{End}_{\mathcal{C}}(T)$ the endomorphism ring of $T$. Then for any additive functor $F: \mathcal{C}^{\mathrm{op}} \rightarrow \mathcal{A} b$, the abelian group $F(T)$ carries a natural left $\Lambda_{T}$-module structure as follows: $\forall \rho \in \Lambda_{T}$ and $\forall x \in F(T), \rho \star x:=F(\rho)(x)$. In particular for any object $C$ in $\mathcal{C}, \mathcal{C}(T, C)$ is a left $\Lambda_{T}$-module with left $\Lambda_{T}$-action: $\rho \star \alpha:=\rho \circ \alpha, \forall \rho \in \Lambda_{T}$ and $\forall \alpha \in \mathcal{C}(T, C)$. Then we have an additive functor 


$$
\mathrm{H}_{T}: \mathcal{C} \longrightarrow \operatorname{Mod}-\Lambda_{T}^{\text {op }}, \quad \mathrm{H}_{T}(C)=\mathcal{C}(T, C) .
$$

Let $\mathrm{m}$ be a maximal left ideal of $\Lambda_{T}$, let $S_{\mathrm{m}}$ be the simple $\Lambda_{T}$-module $\Lambda_{T} / \mathrm{m}$ and let $\mu: S_{\mathfrak{m}} \hookrightarrow I_{\mathfrak{m}}$ be the injective envelope of $S_{\mathfrak{m}}$ in Mod- $\Lambda_{T}^{\text {op }}$. Also let $\mathcal{X}$ be a full additive subcategory of $\mathcal{C}$ which is closed under direct summands and contains $T$. It is easy to see that $\mathcal{X}(-, T)_{\mathfrak{m}}$ is a maximal subfunctor of $\mathcal{X}(-, T)$, where:

$$
\mathcal{X}(-, T)_{\mathfrak{m}}(C):=\{\alpha \in \mathcal{X}(C, T) \mid \forall \beta \in \mathcal{X}(T, C): \beta \circ \alpha \in \mathfrak{m}\} .
$$

Hence the quotient $S_{T, \mathfrak{m}}:=\mathcal{X}(-, T) / \mathcal{X}(-, T)_{\mathfrak{m}}: \mathcal{X}^{\text {op }} \rightarrow \mathcal{A} b$ is a simple functor with the property that $S_{T, \mathfrak{m}}(T)=S_{\mathfrak{m}}$. For convenience from now on we set:

$$
\mathrm{H}_{T, \mathrm{~m}}^{\vee}:=\operatorname{Hom}_{\Lambda_{T}}\left[\mathrm{H}_{T}(-), I_{\mathrm{m}}\right]: \mathcal{C}^{\mathrm{op}} \longrightarrow \mathcal{A} b .
$$

In the sequel we denote by $\operatorname{Max}_{l}(\Lambda), \operatorname{resp} \operatorname{Max}_{r}(\Lambda)$, the set of maximal left, resp. right, ideals of a ring $\Lambda$, and by $\operatorname{Iso}(\mathcal{X})$ the isoclass of objects of a category $\mathcal{X}$. An additive functor $F: \mathcal{X}^{\text {op }} \rightarrow \mathcal{A} b$ is called finitely presented if there exists an exact sequence $\mathcal{X}\left(-, X_{1}\right) \rightarrow \mathcal{X}\left(-, X_{0}\right) \rightarrow F \rightarrow 0$ where the $X_{i}$ are in $\mathcal{X}$. If $\mathcal{X}$ is skeletally small. i.e. Iso $(\mathcal{X})$ is a set, then we denote by Mod$\mathcal{X}$, resp. mod- $\mathcal{X}$, the category of contravariant additive, resp. finitely presented, functors $\mathcal{X}^{\text {op }} \rightarrow \mathcal{A} b$.

PROPOSITION 2.1. Let $\mathcal{X}$ be a skeletally small full subcategory of $\mathcal{C}$ containing $T$.

(i) For any functor $F \in \operatorname{Mod}-\mathcal{X}$, there exists an isomorphism:

$$
\psi: \operatorname{Hom}_{\Lambda_{T}}\left(F(T), I_{\mathfrak{m}}\right) \stackrel{\cong}{\longrightarrow}\left[F, \mathrm{H}_{T, \mathfrak{m}}^{\vee}\right] \text {. }
$$

In particular we have an isomorphism: $\left[\mathrm{H}_{T, \mathrm{~m}}^{\vee}, \mathrm{H}_{T, \mathrm{~m}}^{\vee} \stackrel{\cong}{\longrightarrow} \operatorname{End}_{\Lambda}\left(I_{\mathfrak{m}}\right)\right.$ and the functor $\mathrm{H}_{T, \mathrm{~m}}^{\vee}$ has local endomorphism ring.

(ii) The functor $\mathrm{H}_{T, \mathrm{~m}}^{\vee}$ is an injective object in Mod-X. Moreover the functor $\prod_{T \in \operatorname{Iso}(\mathcal{X})} \prod_{\mathrm{m} \in \operatorname{Max}_{l}\left(\Lambda_{T}\right)} \mathrm{H}_{T, \mathrm{~m}}^{\vee}$ is an injective cogenerator in Mod- $\mathcal{X}$.

(iii) There exists an injective envelope in the abelian category Mod- $\mathcal{X}$ :

$$
\phi: S_{T, \mathrm{~m}} \longrightarrow \mathrm{H}_{T, \mathrm{~m}}^{\vee}=\operatorname{Hom}_{\Lambda_{T}}\left[\mathrm{H}_{T}(-), I_{\mathrm{m}}\right] .
$$

Proof. (i) Assume first that $F$ is finitely presented and let $\mathcal{X}\left(-, X_{1}\right) \rightarrow \mathcal{X}\left(-, X_{0}\right) \rightarrow F \rightarrow 0$ be a finite presentation of $F$. By Yoneda's Lemma we have an exact sequence $0 \rightarrow\left[F, \mathrm{H}_{T, \mathrm{~m}}^{\vee}\right] \rightarrow \mathrm{H}_{T, \mathrm{~m}}^{\vee}\left(X_{0}\right) \rightarrow \mathrm{H}_{T, \mathrm{~m}}^{\vee}\left(X_{1}\right)$. On the other hand the exact sequence $\mathcal{X}\left(T, X_{1}\right) \rightarrow \mathcal{X}\left(T, X_{0}\right) \rightarrow F(T) \rightarrow 0$ of left $\Lambda_{T}$-modules, induces an exact sequence $0 \rightarrow \operatorname{Hom}_{\Lambda_{T}}\left(F(T), I_{\mathrm{m}}\right] \rightarrow$ $\mathrm{H}_{T, \mathfrak{m}}^{\vee}\left(X_{0}\right) \rightarrow \mathrm{H}_{T, \mathfrak{m}}^{\vee}\left(X_{1}\right)$. Hence $\left[F, \mathrm{H}_{T, \mathfrak{m}}^{\vee}\right] \cong \operatorname{Hom}_{\Lambda_{T}}\left(F(T), I_{\mathfrak{m}}\right]$. If $F$ is an arbitrary additive functor, we write $F$ as a filtered colimit of finitely presented functors: $F=\lim F_{i}$. Then we have isomorphisms:

$$
\left[F, \mathrm{H}_{T, \mathrm{~m}}^{\vee}\right]=\left[\lim F_{i}, \mathrm{H}_{T, \mathrm{~m}}^{\vee}\right] \stackrel{\cong}{\leftrightarrows} \lim \left[F_{i}, \mathrm{H}_{T, \mathrm{~m}}^{\vee}\right] \stackrel{\cong}{\lim } \operatorname{Hom}_{\Lambda_{T}}\left(F_{i}(T), I_{\mathrm{m}}\right) \stackrel{\cong}{\rightrightarrows}
$$




$$
\operatorname{Hom}_{\Lambda_{T}}\left(\lim _{\longrightarrow} F_{i}(T), I_{\mathfrak{m}}\right)=\operatorname{Hom}_{\Lambda_{T}}\left(F(T), I_{\mathfrak{m}}\right) .
$$

Setting $F=\mathrm{H}_{T, \mathrm{~m}}^{\vee}$ and using that $\mathrm{H}_{T}(T)=\Lambda_{T}$, we obtain isomorphisms:

$$
\left[\mathrm{H}_{T, \mathfrak{m}}^{\vee}, \mathrm{H}_{T, \mathrm{~m}}^{\vee}\right] \stackrel{\cong}{\rightrightarrows} \operatorname{Hom}_{\Lambda_{T}}\left(\operatorname{Hom}_{\Lambda_{T}}\left(\mathrm{H}_{T}(T), I_{\mathfrak{m}}\right), I_{\mathfrak{m}} \stackrel{\cong}{\rightrightarrows} \operatorname{End}_{\Lambda_{T}}\left(I_{\mathfrak{m}}\right)\right. \text {. }
$$

Finally $\operatorname{End}_{\Lambda_{T}}\left(I_{\mathfrak{m}}\right)$ is local since $I_{\mathfrak{m}}$ is the injective envelope of a simple $\Lambda_{T^{-}}$ module.

(ii) Let $0 \rightarrow F_{1} \rightarrow F_{2} \rightarrow F_{3} \rightarrow 0$ be a short exact sequence in Mod- $\mathcal{X}$. Then we have a short exact sequence $0 \rightarrow F_{1}(T) \rightarrow F_{2}(T) \rightarrow F_{3}(T) \rightarrow 0$ in Mod$\Lambda_{T}^{\mathrm{op}}$. Since $I_{\mathrm{m}}$ is injective, we have a short exact sequence $0 \rightarrow \operatorname{Hom}_{\Lambda_{T}}\left(F_{3}(T), I_{\mathfrak{m}}\right) \rightarrow \operatorname{Hom}_{\Lambda_{T}}\left(F_{2}(T), I_{\mathfrak{m}}\right) \rightarrow \operatorname{Hom}_{\Lambda_{T}}\left(F_{1}(T), I_{\mathfrak{m}}\right) \rightarrow 0 \quad$ in $\mathcal{A b}$. But by (i) the last sequence is isomorphic to $0 \rightarrow\left[F_{3}, \mathrm{H}_{T, \mathrm{~m}}^{\vee}\right] \rightarrow\left[F_{2}, \mathrm{H}_{T, \mathrm{~m}}^{\vee}\right] \rightarrow\left[F_{1}, \mathrm{H}_{T, \mathrm{~m}}^{\vee}\right] \rightarrow 0$. Hence $\mathrm{H}_{T, \mathfrak{m}}^{\vee}$ is an injective functor. Now if $\left[F, \mathrm{H}_{T, \mathrm{~m}}^{\vee}\right]=0$ for any object $T$ in $\mathcal{X}$ and any maximal left ideal m of $\Lambda_{T}$, we have by (i) that $F(T)=0$ for any $T \in \mathcal{X}$. Hence $F=0$.

(iii) Choosing $F=S_{T, m}$ in part (i), we have isomorphisms:

$$
\left[S_{T, \mathfrak{m}}, \mathrm{H}_{T, \mathfrak{m}}^{\vee}\right] \rightarrow \operatorname{Hom}_{\Lambda_{T}}\left(S_{T, \mathfrak{m}}(T), I_{\mathfrak{m}}\right) \rightarrow \operatorname{Hom}_{\Lambda_{T}}\left(S_{\mathfrak{m}}, I_{\mathfrak{m}}\right) .
$$

Since $\operatorname{Hom}_{\Lambda_{T}}\left(S_{\mathfrak{m}}, I_{\mathfrak{m}}\right) \neq 0$, there exists a non-zero morphism $\phi: S_{T, \mathfrak{m}} \rightarrow \mathrm{H}_{T, \mathfrak{m}}^{\vee}$ which is an injective envelope since $\mathrm{H}_{T, \mathrm{~m}}^{\vee}$ is injective with local endomorphism ring.

For later reference we describe explicitly the injective envelope $\phi: S_{T, \mathfrak{m}} \longmapsto \mathrm{H}_{T, \mathfrak{m}}^{\vee}$. Let $\mu: S_{\mathfrak{m}} \hookrightarrow I_{\mathfrak{m}}$ be the injective envelope of $S_{\mathfrak{m}}$ in Mod- $\Lambda_{T}^{\mathrm{op}}$. Then for any object $X \in \mathcal{X}$, any element $x \in S_{T, \mathrm{~m}}(X)$ and any morphism $y: T \rightarrow X$, the morphism $\phi_{X}: S_{T, \mathfrak{m}}(X) \longmapsto \mathrm{H}_{T, \mathrm{~m}}^{\vee}(X)$ acts as follows: $\phi_{X}(x)(y)=\mu\left(\widetilde{x}_{T}(y)\right)$, where $\widetilde{x}: \mathcal{X}(-, X) \rightarrow S_{T, \mathfrak{m}}$ is the unique morphism such that $\widetilde{x}_{X}\left(1_{X}\right)=x$.

\subsection{REPRESENTABILITY OF FUNCTORS AND CONSTRUCTION OF MORPHISMS}

It will be crucial in the sequel to have conditions ensuring that the functor $\mathrm{H}_{T, \mathrm{~m}}^{\vee}$ is representable in $\mathcal{C}$. In this subsection we discuss briefly some consequences of this fact which allow us to construct morphisms in $\mathcal{C}$.

Let $I$ be a left $\Lambda_{T}$-module and consider the functor:

$$
{ }_{I} \mathrm{H}_{T}^{\vee}:=\operatorname{Hom}_{\Lambda_{T}}\left[\mathrm{H}_{T}(-), I\right]: \mathcal{C}^{\mathrm{op}} \longrightarrow \mathcal{A} b \text {. }
$$

From now on we assume that ${ }_{I} \mathrm{H}_{T}^{\vee}$ is representable with representing object $\mathbb{D}^{I}(T)$ which we call the I-dual object of $T$. Hence we have a natural isomorphism:

$$
\omega: \mathcal{C}\left(-, \mathbb{D}^{I}(T)\right) \stackrel{\cong}{\rightarrow}{ }_{I} \mathrm{H}_{T}^{\vee}=\operatorname{Hom}_{\Lambda_{T}}\left[\mathrm{H}_{T}(-), I\right] .
$$

For any morphism $\alpha: A \rightarrow \mathbb{D}^{I}(T)$ in $\mathcal{C}$ we have the following commutative diagram: 


$$
\begin{array}{ccc}
\mathcal{C}\left(\mathbb{D}^{I}(T), \mathbb{D}^{I}(T)\right) & \stackrel{\omega_{\mathbb{D}^{I}(T)}}{\longrightarrow} & \operatorname{Hom}_{\Lambda_{T}}\left[\mathrm{H}_{T}\left(\mathbb{D}^{I}(T)\right), I\right] \\
\alpha_{*} \downarrow & \mathrm{H}_{T}(\alpha)_{*} \downarrow \\
\mathcal{C}\left(A, \mathbb{D}^{I}(T)\right) & \stackrel{\omega_{A}}{\longrightarrow} & \operatorname{Hom}_{\Lambda_{T}}\left[\mathrm{H}_{T}(A), I\right]
\end{array}
$$

where $\alpha_{*}=\mathcal{C}\left(\alpha, \mathbb{D}^{I}(T)\right)$ and $\mathrm{H}_{T}(\alpha)_{*}=\operatorname{Hom}_{\Lambda_{T}}\left[\mathrm{H}_{T}(\alpha), I\right]$. It follows from (1) that $\mathrm{H}_{T}(\alpha) \circ \omega_{\mathbb{D}^{I}(T)}\left(1_{\mathbb{D}^{I}(T)}\right)=\omega_{A}(\alpha)$. In the following we collect some properties of the map $\omega_{\mathbb{D}^{I}(T)}\left(1_{\mathbb{D}^{I}(T)}\right): \mathrm{H}_{T}\left(\mathbb{D}^{I}(T)\right) \rightarrow I$ which will be useful later.

\section{LEMMA 2.2.}

(1) The map $\omega_{\mathbb{D}^{I}(T)}\left(1_{\mathbb{D}^{I}(T)}\right): \mathrm{H}_{T}\left(\mathbb{D}^{I}(T)\right) \rightarrow$ I is invertible.

(2) The canonical map $\mathcal{C}\left(-, \mathbb{D}^{I}(T)\right) \rightarrow \operatorname{Hom}_{\Lambda_{T}}\left[\mathrm{H}_{T}(-), \mathrm{H}_{T}\left(\mathbb{D}^{I}(T)\right)\right]$ is invertible.

(3) There exists an isomorphism of rings: $\operatorname{End}_{\mathcal{C}}\left(\mathbb{D}^{I}(T)\right) \cong \operatorname{End}_{\Lambda_{T}}(I)$.

Proof. Using the commutativity of diagram (1), an easy calculation shows that: $\omega_{T}(\alpha)\left(1_{T}\right)=\omega_{\mathbb{D}^{I}(T)}\left(1_{\mathbb{D}^{I}(T)}\right)(\alpha)$, for any map $\alpha: T \rightarrow \mathbb{D}^{I}(T)$ in $\mathcal{C}$. This implies that $\omega_{\mathbb{D}^{I}(T)}\left(1_{\mathbb{D}^{I}(T)}\right)=\omega_{T} \circ \psi$, where $\psi$ is the canonical isomorphism $\operatorname{Hom}_{\Lambda_{T}}\left[\mathrm{H}_{T}(T), I \stackrel{\cong}{\rightarrow} I, \psi: \rho \mapsto \rho\left(1_{T}\right)\right.$. In particular $\omega_{\mathbb{D}^{I}(T)}\left(1_{\mathbb{D}^{I}(T)}\right)$ is invertible. Then for any map $\alpha: A \rightarrow \mathbb{D}^{I}(T)$ in $\mathcal{C}$, we have $\mathrm{H}_{T}(\alpha)=\omega_{\mathbb{D}^{I}(T)}\left(1_{\mathbb{D}^{I}(T)}\right)^{-1}\left(\omega_{A}(\alpha)\right)$. Therefore the canonical map $\alpha \mapsto \mathrm{H}_{T}(\alpha)$ is equal to the composition $\omega_{A} \circ \omega_{\mathbb{D}^{I}(T)}^{-1}\left(1_{\mathbb{D}^{I}(T)}\right)$ and consequently it is invertible. The last assertion follows from the isomorphisms: $\operatorname{End}_{\mathcal{C}}\left(\mathbb{D}^{I}(T)\right) \stackrel{\cong}{\rightrightarrows}$ $\operatorname{Hom}_{\Lambda_{T}}\left[\mathrm{H}_{T}\left(\mathbb{D}^{I}(T)\right), I\right] \stackrel{\cong}{\rightrightarrows} \operatorname{Hom}_{\Lambda_{T}}(I, I)=\operatorname{End}_{\Lambda_{T}}(I)$.

Now let $I=I_{\mathfrak{m}}$ be the injective envelope of the simple left $\Lambda_{T}$-module $S_{\mathfrak{m}}:=\Lambda_{T} / \mathfrak{m}$, where $\mathrm{m}$ is a maximal left ideal of $\Lambda_{T}$.

DEFINITION 2.3. If the functor

$$
\mathrm{H}_{T, \mathrm{~m}}^{\vee}=\operatorname{Hom}_{\Lambda_{T}}\left[\mathrm{H}_{T}(-), I_{\mathrm{m}}\right]: \mathcal{C}^{\mathrm{op}} \longrightarrow \mathcal{A} b
$$

is representable, then the representing object of $\mathrm{H}_{T, \mathfrak{m}}^{\vee}$ is denoted by $\mathbb{D}_{\mathfrak{m}}(T)$, i.e. $\mathbb{D}_{\mathfrak{m}}(T)=\mathbb{D}^{I_{\mathfrak{m}}}(T)$, and, by abuse of language, is called the $\mathrm{m}$-dual object of $T$ in $\mathcal{C}$ with respect to the maximal left ideal $\mathrm{m}$. In this case we denote always by $\omega: \mathcal{C}\left(-, \mathbb{D}_{\mathfrak{m}}(T)\right) \stackrel{\cong}{\rightarrow} \mathrm{H}_{T, \mathfrak{m}}^{\vee}$ the associated natural isomorphism.

Remark 2.4. In the sequel when we consider the functor $\mathrm{H}_{T, \mathrm{~m}}^{\vee}$ we implicitly assume without further mentioning that an object $T$ is given in $\mathcal{C}$ with endomorphism ring $\Lambda_{T}, \mathrm{~m}$ is a maximal ideal of $\Lambda_{T}$ and $I_{\mathrm{m}}$ is the injective envelope of the simple left $\Lambda_{T}$-module $S_{\mathfrak{m}}:=S_{T, \mathfrak{m}}(T)$, where $S_{T, \mathfrak{m}}$ is the simple functor $\mathcal{C}(-, T) / \mathcal{C}(-, T)_{\mathfrak{m}}$. 
Assume now that the m-dual object $\mathbb{D}_{\mathfrak{m}}(T)$ of $T$ exists. Consider the composition $\varepsilon \circ \mu: \Lambda_{T}=\mathrm{H}_{T}(T) \rightarrow S_{\mathfrak{m}} \longmapsto I_{\mathfrak{m}}$, where $\varepsilon: \Lambda_{T} \rightarrow S_{\mathfrak{m}}$ is the canonical projection and $\mu: S_{\mathfrak{m}} \longmapsto I_{\mathfrak{m}}$ is the injective envelope of $S_{\mathfrak{m}}$, and we set

$$
h:=\omega_{T}^{-1}(\varepsilon \circ \mu): T \longrightarrow \mathbb{D}_{\mathfrak{m}}(T) .
$$

We now summarize some basic properties of $h$ which will be useful later.

\section{LEMMA 2.5.}

(1) The morphism $h$ is non-zero and for any morphism $\alpha: \mathbb{D}_{\mathfrak{m}}(T) \rightarrow C$ in $\mathcal{C}$ which is not a split monomorphism, it holds: $h \circ \alpha=0$.

(2) The map $\mathrm{H}_{T}(h): \mathrm{H}_{T}(T) \rightarrow \mathrm{H}_{T}\left(\mathbb{D}_{\mathfrak{m}}(T)\right.$ ) has image $\operatorname{ImH}_{T}(h)=S_{\mathfrak{m}}$ and the inclusion $\operatorname{ImH}_{T}(h) \hookrightarrow \mathrm{H}_{T}\left(\mathbb{D}_{\mathfrak{m}}(T)\right)$ is an injective envelope of $S_{\mathfrak{m}}$.

(3) The map $\omega_{\mathbb{D}_{\mathfrak{m}}(T)}\left(1_{\mathbb{D}_{\mathfrak{m}}(T)}\right): \mathrm{H}_{T}\left(\mathbb{D}_{\mathfrak{m}}(T)\right) \rightarrow I_{\mathfrak{m}}$ is invertible and induces a ring isomorphism $\operatorname{End}_{\mathcal{C}}\left(\mathbb{D}_{\mathfrak{m}}(T)\right) \stackrel{\cong}{\rightarrow} \operatorname{End}_{\Lambda_{T}}\left(I_{\mathfrak{m}}\right)$. In particular $\operatorname{End}_{\mathcal{C}}\left(\mathbb{D}_{\mathfrak{m}}(T)\right)$ is local.

(4) The pairing $\mathcal{C}(T, A) \times \mathcal{C}\left(A, \mathbb{D}_{\mathfrak{m}}(T)\right) \rightarrow I_{\mathfrak{m}},(f, g) \mapsto \omega^{-1}(f \circ g)\left(1_{T}\right)$ is nondegenerate.

Proof. (1) By construction $h \neq 0$. Let $\alpha: \mathbb{D}_{\mathfrak{m}}(T) \rightarrow C$ be a morphism in $\mathcal{C}$ which is not a split monomorphism. If $\mathrm{H}_{T}(\alpha): \mathrm{H}_{T}\left(\mathbb{D}_{\mathfrak{m}}(T)\right) \rightarrow \mathrm{H}_{T}(C)$ is a monomorphism, then $\mathrm{H}_{T}(\alpha)$ splits since $\mathrm{H}_{T}\left(\mathbb{D}_{\mathfrak{m}}(T)\right) \stackrel{\cong}{\rightrightarrows} I_{\mathfrak{m}}$ is injective. Then by using part (2) of Lemma 2.2 we see easily that $\alpha$ splits and this is not the case. Since $\operatorname{ImH}_{T}(h)=S_{\mathfrak{m}}$, this implies that $\mathrm{H}_{T}(h) \circ \mathrm{H}_{T}(\alpha)=0$ and therefore $h \circ \alpha=0$. Parts (2), (3) and (4) follow directly from part (1) and Lemma 2.2 .

There is a useful connection between existence of m-dual objects and existence of (co)generating sets. Recall that a set of objects $\mathcal{X}$ in $\mathcal{C}$ is a generating, resp. cogenerating, set, in $\mathcal{C}$, if $\mathcal{C}(T, C)=0$, resp. $\mathcal{C}(C, T)=0$, $\forall T \in \mathcal{X}$, implies $C=0$.

COROLLARY 2.6. Assume that $\mathcal{C}$ contains a set of objects $\mathcal{X}$ with the property that for any object $T$ in $\mathcal{X}$ and any maximal left ideal $\mathrm{m}$ of $\Lambda_{T}$, the functor $\mathrm{H}_{T, \mathrm{~m}}^{\vee}: \mathcal{C} \rightarrow \mathcal{A} b$ is representable. Then we have the following.

(i) The set $\left\{\mathrm{H}_{T, \mathrm{~m}}^{\vee} \mid T \in \mathcal{X}, \mathrm{m} \in \operatorname{Max}_{l}\left(\Lambda_{T}\right)\right\}$ is a cogenerating set of indecomposable injectives in the module category Mod- $\mathcal{X}$.

(ii) The set of objects $\mathcal{X}$ is a generating set in $\mathcal{C}$ if and only if the set of objects $\left\{\mathbb{D}_{\mathfrak{m}}(T) \mid T \in \mathcal{X}, \mathfrak{m} \in \operatorname{Max}_{l}\left(\Lambda_{T}\right)\right\}$ is a cogenerating set in $\mathcal{C}$.

\subsection{REALIZABILITY OF INJECTIVES}

Let as before $T$ be an object in $\mathcal{C}$. If $I$ is an injective left $\Lambda_{T}$-module, then the above results suggest that there should be a connection between 
representability of the functor ${ }_{I} \mathrm{H}_{T}^{\vee}$ and realizability of the $\Lambda_{T}$-module $I$ in the sense of the following definition.

DEFINITION 2.7. Let $E$ be an object in $\mathcal{C}$ and $I$ a left $\Lambda_{T}$-module. We say that $I$ is $T$-realizable by $E$ if the following conditions hold.

(i) There exists an isomorphism $\mathrm{H}_{T}(E)=\mathcal{C}(T, E) \cong I$.

(ii) The canonical map $\mathcal{C}(-, E) \rightarrow \operatorname{Hom}_{\Lambda_{T}}\left(\mathrm{H}_{T}(-), \mathrm{H}_{T}(E)\right)$ is surjective.

(iii) The canonical map $\mathcal{C}(E, E) \rightarrow \operatorname{Hom}_{\Lambda_{T}}\left(\mathrm{H}_{T}(E), \mathrm{H}_{T}(E)\right)$ is injective.

In this case we say that the object $E$ is a T-realization of $I$.

In the triangulated case we have the following connection between representability of functors and realizability of injective modules.

PROPOSITION 2.8. Let $\mathcal{C}$ be a triangulated category and let $T$ be an object of $\mathcal{C}$. If $I$ is an injective left $\Lambda_{T}$-module, then the following statements are equivalent.

(i) The left $\Lambda_{T}$-module I is T-realizable.

(ii) The functor ${ }_{I} \mathrm{H}_{T}^{\vee}:=\operatorname{Hom}_{\Lambda_{T}}\left[\mathrm{H}_{T}(-), I\right]: \mathcal{C}^{\mathrm{op}} \rightarrow \mathcal{A} b$ is representable.

Proof. Part (ii) $\Rightarrow$ (i) follows from Lemma 2.2. Assume that $I$ is $T$-realizable and let $E$ be a $T$-realization of $I$. If $\alpha: C \rightarrow E$ is a morphism in $\mathcal{C}$ such that $\mathrm{H}_{T}(\alpha)=0$, then for any morphism $\rho: T \rightarrow C$ in $\mathcal{C}$ we have: $\rho \circ \alpha=0$. Let $\beta: E \rightarrow D$ be the cofiber of $\alpha$. Since $\mathrm{H}_{T}(\alpha)=0$, it follows that $\mathrm{H}_{T}(\beta): \mathrm{H}_{T}(E) \rightarrow \mathrm{H}_{T}(D)$ is a monomorphism. Since the left $\Lambda_{T}$-module $\mathrm{H}_{T}(E) \cong I$ is injective, there exists a map $\widetilde{\delta}: \mathrm{H}_{T}(D) \rightarrow \mathrm{H}_{T}(\underset{\delta}{E})$ such that $\mathrm{H}_{T}(\beta) \circ \widetilde{\delta}=1_{\mathrm{H}_{T}(E)}$. By condition (ii) of Definition 2.7, we have $\widetilde{\delta}=\mathrm{H}_{T}(\delta)$ for some morphism $\delta: D \rightarrow E$, and then by condition (iii) we have $\beta \circ \delta=1_{E}$. Hence $\beta$ is split monic and therefore $\alpha=0$. We infer that the canonical map $\mathcal{C}(-, E) \rightarrow \operatorname{Hom}_{\Lambda_{T}}\left(\mathrm{H}_{T}(-), \mathrm{H}_{T}(E)\right)$ is invertible. Composing this natural isomorphism with the natural isomorphism induced by the isomorphism $\mathrm{H}_{T}(E) \stackrel{\cong}{\rightrightarrows} I$, we get an isomorphism $\mathcal{C}(-, E) \stackrel{\cong}{\rightarrow} \operatorname{Hom}_{\Lambda_{T}}\left(\mathrm{H}_{T}(-), I\right)$.

COROLLARY 2.9. Let $\mathcal{C}$ be a triangulated category. Let $T$ be an object in $\mathcal{C}$ with endomorphism ring $\Lambda_{T}$ and let $\mathrm{m}$ be a maximal left ideal of $\Lambda_{T}$. Then the functor $\mathrm{H}_{T, \mathrm{~m}}^{\vee}=\operatorname{Hom}_{\Lambda_{T}}\left[\mathrm{H}_{T}(-), I_{\mathrm{m}}\right]$ is representable, that is the $\mathrm{m}$-dual object $\mathbb{D}_{\mathfrak{m}}(T)$ exists, if and only if the injective envelope $I_{\mathfrak{m}}$ of the simple module $\Lambda_{T} / \mathrm{m}$ is T-realizable.

\section{Representability of Functors and Auslander-Reiten Triangles}

Our aim in this section is to prove some general existence results for Auslander-Reiten triangles in a sufficiently general triangulated category. The basic tool is the construction of morphisms arising from the 
representability of the injective envelopes of the simple contravariant functors.

Let $\mathcal{C}$ be an additive category. We recall from [2] that a morphism $f: B \rightarrow C$ is called right almost split in $\mathcal{C}$ if $f$ is not a split epimorphism and any morphism $\alpha: E \rightarrow C$ which is not a split epimorphism factors through $f$. It is easy to see that the target of a right almost split morphism has local endomorphism ring, see [7]. A morphism $f: B \rightarrow C$ in $\mathcal{C}$ is called right minimal [10] if any endomorphism $\alpha: B \rightarrow B$ such that $\alpha \circ f=f$, is an automorphism. A minimal right almost split morphism is a right minimal right almost split morphism. The notions of left almost split, left minimal and minimal left almost split morphisms are defined dually. Recall from [7] that an exact sequence $(E): 0 \rightarrow E \stackrel{g}{\rightarrow} A \stackrel{f}{\rightarrow} T \rightarrow 0$ in an abelian category, or more generally in an exact category in the sense of Quillen [73], is called an Auslander-Reiten sequence, or AR-sequence for short, or almost split sequence, if $f$ is right almost split and $g$ is left almost split. We refer to [4,7] and the more accessible [13] for basic information on Auslander-Reiten sequences and the decisive role they play in representation theory.

From now on we assume that $\mathcal{C}$ is a triangulated category with suspension functor $\Sigma$. The following important concept was introduced by Happel [35] in order to develop Auslander-Reiten theory in triangulated categories.

DEFINITION 3.1. A triangle $(T): A \stackrel{g}{\rightarrow} B \stackrel{f}{\rightarrow} C \stackrel{h}{\rightarrow} \Sigma A$ in $\mathcal{C}$ is called an $A u s$ lander-Reiten triangle, $A R$-triangle for short, if $g$ is left almost split and $f$ is right almost split. In this case we use the following notations: $\tau^{+}(C)=A$ and $\tau^{-}(A)=C$ and we call $\tau^{+}, \tau^{-}$the Auslander-Reiten operators.

In what follows we need the following useful characterizations of AR-triangles.

LEMMA 3.2. [1, Theorem 2.4], [35, Section 3] For a triangle $(T)$ as above, the following statements are equivalent.

(i) $(T)$ is an AR-triangle.

(ii) $g$ is minimal left almost split.

(iii) $f$ is minimal right almost split.

(iv) $g$ is left almost split and $\operatorname{End}(C)$ is local.

(v) $f$ is left almost split and $\operatorname{End}(A)$ is local.

It is easy to see that an AR-triangle is uniquely determined up to isomorphism by its end terms, in the following sense. If $\left(T_{i}\right): A_{i} \rightarrow B_{i} \rightarrow C_{i} \rightarrow \Sigma\left(A_{i}\right)$ are AR-triangles, $i=1,2$, then $A_{1} \cong A_{2}$ iff $C_{1} \cong C_{2}$ iff the triangles $\left(T_{i}\right)$ are isomorphic.

DEFINITION 3.3. [36]. C has right, resp. left, AR-triangles, if for any object $C$, resp. $A$, with local endomorphism ring, there exists an AR-triangle 
$A \stackrel{g}{\rightarrow} B \stackrel{f}{\rightarrow} \mathcal{C} \stackrel{h}{\rightarrow} \Sigma(A)$ in $\mathcal{C}$. And $\mathcal{C}$ has $A R$-triangles, if $\mathcal{C}$ has left and right AR-triangles.

Let $T$ be an object in $\mathcal{C}$ with endomorphism $\operatorname{ring} \Lambda_{T}=\operatorname{End}_{\mathcal{C}}(T)$. As in section 2 we have the functors $\mathrm{H}_{T}: \mathcal{C} \rightarrow \operatorname{Mod}-\Lambda_{T}^{\mathrm{op}}, \mathrm{H}_{T}(C)=\mathcal{C}(T, C)$, and $\mathrm{H}_{T, \mathrm{~m}}^{\vee}:=\operatorname{Hom}_{\Lambda_{T}}\left[\mathrm{H}_{T}(-), I_{\mathrm{m}}\right]: \mathcal{C}^{\text {op }} \rightarrow \mathcal{A} b$, where $\mathrm{m}$ is a maximal left ideal of $\Lambda_{T}$ and $I_{\mathfrak{m}}$ is the injective envelope of the simple left $\Lambda_{T}$-module $S_{\mathfrak{m}}:=\Lambda_{T} / \mathrm{m}$.

The following basic result shows that existence of AR-triangles in $\mathcal{C}$ is related to representability of the indecomposable injective functors $\mathrm{H}_{T, \mathfrak{m}}^{\vee}$. Note that representability of $\mathrm{H}_{T, \mathrm{~m}}^{\vee}$ is equivalent to the existence of the m-dual object $\mathbb{D}_{\mathfrak{m}}(T)$ of $T$. First recall that an additive category is called a KrullSchmidt category if any of its objects is a finite coproduct of objects with local endomorphism ring.

THEOREM 3.4. If the functor $\mathrm{H}_{T, \mathrm{~m}}^{\vee}$ is representable, then we have the following.

(i) $\mathbb{D}_{\mathfrak{m}}(T)$ is the source of a left almost split morphism in $\mathcal{C}$.

(ii) The $\mathrm{m}$-dual object $\mathbb{D}_{\mathfrak{m}}(T)$ of $T$ is the source of an AR-triangle in $\mathcal{C}$ provided that one of the following conditions hold:

(a) $\mathcal{C}$ is a Krull-Schmidt category.

(b) Thas local endomorphism ring.

Proof. (i) Let $h: T \rightarrow \mathbb{D}_{\mathfrak{m}}(T)$ be the morphism constructed in Section 2 and let $T \stackrel{h}{\rightarrow} \mathbb{D}_{\mathfrak{m}}(T) \stackrel{g}{\rightarrow} A \rightarrow \Sigma(T)$ be a triangle in $\mathcal{C}$. By Lemma $2.5, g$ is not a split monomorphism and any morphism $\alpha: \mathbb{D}_{\mathfrak{m}}(T) \rightarrow B$ which is not a split monomorphism factors through $g$. This shows that $g$ is left almost split.

(ii) If $\Lambda_{T}$ is local, then the assertion follows from part (i) and Lemma 3.2. If $\mathcal{C}$ is Krull-Schmidt, then it is well-known that there exists a decomposition $A=A_{1} \oplus A_{2}$ such that $g=\left(g_{1}, 0\right): \mathbb{D}_{\mathfrak{m}}(T) \rightarrow A_{1} \oplus A_{2}$ and $g_{1}: \mathbb{D}_{\mathfrak{m}}(T) \rightarrow A_{1}$ is left minimal, see [60, Proposition 1.2]. Clearly $g_{1}$ is minimal left almost split, so any triangle $\mathbb{D}_{\mathfrak{m}}(T) \stackrel{g_{1}}{\rightarrow} A_{1} \rightarrow T_{1} \rightarrow \Sigma \mathbb{D}_{\mathfrak{m}}(T)$ is an AR-triangle in $\mathcal{C}$, by Lemma 3.2.

COROLLARY 3.5. Let $\mathcal{C}$ be a triangulated category and let $T$ be an object in $\mathcal{C}$ with local endomorphism ring $\Lambda_{T}$. If the functor

$$
\mathrm{H}_{T, \mathrm{~m}}^{\vee}:=\operatorname{Hom}_{\Lambda_{T}}\left[\mathrm{H}_{T}(-), I_{\mathrm{m}}\right]: \mathcal{C}^{\mathrm{op}} \rightarrow \mathcal{A} b
$$

is representable, then there exists an AR-triangle $\Sigma^{-1} \mathbb{D}_{\mathfrak{m}}(T) \rightarrow A \rightarrow T \rightarrow E$ in $\mathcal{C}$, where $\mathbb{D}_{\mathfrak{m}}(T)$ is the representing object of the functor $\mathrm{H}_{T, \mathfrak{m}}^{\vee}$.

The above result raises the question if, conversely, the existence of an AR-triangle $\Sigma^{-1} E \rightarrow A \rightarrow T \rightarrow E$ in $\mathcal{C}$ implies the representability of the functor $\mathrm{H}_{T, \mathrm{~m}}^{\vee}$. We devote the rest of this section to an analysis of this question. We begin with the following preliminary result which shows that 
the morphism $T \rightarrow E$ above satisfies some special properties which will be needed later.

LEMMA 3.6. Let $\Sigma^{-1} E \stackrel{g}{\rightarrow} A \stackrel{f}{\rightarrow} T \stackrel{h}{\rightarrow} E$ be an AR-triangle in $\mathcal{C}$.

(i) The canonical map $\mathcal{C}(-, E) \rightarrow \operatorname{Hom}_{\Lambda_{T}}\left(\mathrm{H}_{T}(-), \mathrm{H}_{T}(E)\right)$ is injective.

(ii) $S_{\mathfrak{m}}=\Lambda_{T} \cdot h=\operatorname{ImH}_{T}(h)=\operatorname{SocH}_{T}(E)$.

(iii) There are essential inclusions $S_{\mathfrak{m}} \hookrightarrow \mathrm{H}_{T}(E) \hookrightarrow I_{\mathfrak{m}}$.

(iv) The module $\mathrm{H}_{T}(E)$ is injective if and only if $\mathrm{H}_{T}(E)=I_{\mathfrak{m}}$.

Proof. We prove only part (i) leaving the easy proof of the other parts to the reader. Let $\alpha: C \rightarrow E$ be a morphism in $\mathcal{C}$ such that $\mathrm{H}_{T}(\alpha)=0$. Then $\rho \circ \alpha=0$ for any morphism $\rho: T \rightarrow C$. If $\alpha \neq 0$, then the cofiber $\beta: E \rightarrow D$ of $\alpha$ is not a split monomorphism. Since $\Sigma(g)$ is left almost split, $\beta$ factors through $\Sigma(g)$. This implies that $h \circ \beta=0$. Hence there exists $\rho: T \rightarrow C$ such that $\rho \circ \alpha=h$. Since $\rho \circ \alpha=0$, it follows that $h=0$ and this is impossible. Hence $\alpha=0$ and the map $\mathcal{C}(C, E) \rightarrow \operatorname{Hom}_{\Lambda_{T}}\left(\mathrm{H}_{T}(C), \mathrm{H}_{T}(E)\right)$ is injective.

We are interested in finding sufficient conditions ensuring that the canonical maps in Lemma 3.6 are invertible and also when the module $\mathrm{H}_{T}(E)$ is injective. First recall from [10] that a subcategory $\mathcal{X}$ of an additive category $\mathcal{C}$ is called contravariantly finite if for any object $C$ in $\mathcal{C}$ there exists a morphism $f_{C}: X_{C} \rightarrow C$ with $X_{C}$ in $\mathcal{X}$ such that any morphism $X \rightarrow C$ with $X$ in $\mathcal{X}$ factors through $f_{C}$. In this case $f_{C}$ is called a right $\mathcal{X}$-approximation of $C$. The dual notions are covariantly finite and left approximation, and $\mathcal{X}$ is called functorially finite if $\mathcal{X}$ is both contravariantly and covariantly finite. We denote by $\operatorname{add}(\mathcal{X})$ the full subcategory of $\mathcal{C}$ consisting of all direct summands of finite direct sums of objects from $\mathcal{X}$. An additive category $\mathcal{C}$ is $R$-linear over a commutative ring $R$, if $\mathcal{C}(A, B)$ is an $R$-module, $\forall A, B \in \mathcal{C}$, and the composition of morphisms is $R$-bilinear.

LEMMA 3.7. Let $\mathcal{C}$ be an $R$-linear category over a commutative ring $R$ and let $T$ be an object in $\mathcal{C}$. Then the following are equivalent:

(i) $\operatorname{add}(T)$ is contravariantly finite in $\mathcal{C}$.

(ii) For any object $C$ in $\mathcal{C}$, the left $\Lambda_{T}$-module $\mathcal{C}(T, C)$ is finitely generated.

(iii) For any $C$ in $\mathcal{C}$, the $R$-module $\mathcal{C}(T, C)$ is finitely generated.

If (i) holds, then the functor $\mathrm{H}_{T}$ induces an equivalence between $\operatorname{add}(T)$ and the category $\mathcal{P}_{\Lambda_{T}}$ of finitely generated projective $\Lambda_{T}$-modules, and the canonical map $\mathcal{C}(X, C) \rightarrow \operatorname{Hom}_{\Lambda_{T}}\left(\mathrm{H}_{T}(X), \mathrm{H}_{T}(E)\right)$ is invertible, $\forall X \in \operatorname{add}(T), \forall E \in \mathcal{C}$.

Proof. The equivalences follow from Proposition 1.9 of [8]. The final assertion is standard and its proof is left to the reader. 
Recall that a module $X$ over a ring $\Lambda$ is called FP-injective if $\operatorname{Ext}_{\Lambda}^{1}(F, X)=0$ for any finitely presented module $F$. Note that $\Lambda$ is left Noetherian if and only if any left FP-injective module is injective.

LEMMA 3.8. Let $\Sigma^{-1} E \rightarrow A \rightarrow T \rightarrow E$ be an $A R$-triangle in $\mathcal{C}$. If $\operatorname{add}(T)$ is contravariantly finite in $\mathcal{C}$, then the left $\Lambda_{T}$-module $\mathrm{H}_{T}(E)$ is FP-injective and the canonical map $\mathcal{C}(-, E) \rightarrow \operatorname{Hom}_{\Lambda_{T}}\left(\mathrm{H}_{T}(-), \mathrm{H}_{T}(E)\right)$ is invertible. In particular the ring $\operatorname{End}_{\Lambda_{T}}\left(\mathrm{H}_{T}(E)\right)$ is local.

Proof. By Lemma 3.6 it suffices to show that any map $\widetilde{\alpha}: \mathrm{H}_{T}(C) \rightarrow \mathrm{H}_{T}(E)$ is of the form $\mathrm{H}_{T}(\alpha)$ for some morphism $\alpha: C \rightarrow E$. Since $\operatorname{add}(T)$ is contravariantly finite in $\mathcal{C}$, there exists a triangle $K \stackrel{\phi}{\rightarrow} T_{0} \stackrel{\psi}{\rightarrow} C \rightarrow \Sigma(K)$ where $\psi: T_{0} \rightarrow C$ is a right add $(T)$-approximation of $C$. By Lemma 3.7 there exists a morphism $\beta: T_{0} \rightarrow E$ such that $\mathrm{H}_{T}(\psi) \circ \widetilde{\alpha}=\mathrm{H}_{T}(\beta)$. Consider the morphism $\phi \circ \beta: K \rightarrow E$. Then obviously $\mathrm{H}_{T}(\phi \circ \beta)=0$. Then by Lemma 3.6 we have $\phi \circ \beta=0$. Hence there exists a morphism $\alpha: C \rightarrow E$ such that $\psi \circ \alpha=\beta$. Then $\mathrm{H}_{T}(\psi) \circ \mathrm{H}_{T}(\alpha)=\mathrm{H}_{T}(\beta)=\mathrm{H}_{T}(\psi) \circ \widetilde{\alpha}$. Since $\mathrm{H}_{T}(\psi)$ is an epimorphism, we infer that $\mathrm{H}_{T}(\alpha)=\widetilde{\alpha}$.

Now let $F$ be a finitely presented left $\Lambda_{T}$-module. By Lemma 3.7 a finite presentation of $F$ is of the form $\mathrm{H}_{T}\left(T_{1}\right) \stackrel{\mathrm{H}_{T}(\alpha)}{\longrightarrow} \mathrm{H}_{T}\left(T_{0}\right) \rightarrow F \rightarrow 0$, where $\alpha: T_{1} \rightarrow T_{0}$ is a morphism between objects in $\operatorname{add}(T)$. Let $C \stackrel{\sigma}{\rightarrow} T_{1} \stackrel{\alpha}{\rightarrow} T_{0} \rightarrow \Sigma(C)$ be a triangle in $\mathcal{C}$, and let $K \stackrel{\tau}{\rightarrow} T_{2} \stackrel{\rho}{\rightarrow} C \stackrel{\kappa}{\rightarrow} \Sigma(K)$ be a triangle in $\mathcal{C}$ where $\rho: T_{2} \rightarrow C$ is a $\operatorname{right} \operatorname{add}(T)$-approximation of $C$. Then we have an exact sequence $\mathrm{H}_{T}(C) \stackrel{\mathrm{H}_{T}(\sigma)}{\longrightarrow} \mathrm{H}_{T}\left(T_{1}\right) \stackrel{\mathrm{H}_{T}(\alpha)}{\longrightarrow} \mathrm{H}_{T}\left(T_{0}\right) \rightarrow F \rightarrow 0$ in Mod- $\Lambda_{T}^{\mathrm{op}}$. Setting $M:=\mathrm{KerH}_{T}(\alpha)$, we have an exact sequence $\mathrm{H}_{T}\left(T_{2}\right) \stackrel{\mathrm{H}_{T}(\rho \circ \sigma)}{\longrightarrow} \mathrm{H}_{T}\left(T_{1}\right) \stackrel{\mathrm{H}_{T}(\alpha)}{\longrightarrow} \mathrm{H}_{T}\left(T_{0}\right) \rightarrow F \rightarrow 0$ which is the beginning of a projective resolution of $F$. Applying the functor $\operatorname{Hom}_{\Lambda_{T}}\left[-, \mathrm{H}_{T}(E)\right]$ and using that the canonical map $\mathcal{C}(-, E) \rightarrow \operatorname{Hom}_{\Lambda_{T}}\left(\mathrm{H}_{T}(-), \mathrm{H}_{T}(E)\right)$ is invertible, we have a complex $(*): \mathcal{C}\left(T_{0}, E\right) \stackrel{\mathcal{C}(\alpha, E)}{\longrightarrow} \mathcal{C}\left(T_{1}, E\right) \stackrel{\mathcal{C}(\rho \circ \sigma, E)}{\longrightarrow} \mathcal{C}\left(T_{2}, E\right)$ the homology of which computes the extension $\operatorname{Ext}_{\Lambda_{T}}^{1}\left[F, \mathrm{H}_{T}(E)\right]$. Let $\zeta: T_{1} \rightarrow E$ be a morphism in $\operatorname{KerC}(\rho \circ \sigma, E)$. Then $\rho \circ \sigma \circ \zeta=0$, hence $\sigma \circ \zeta=\kappa \circ \xi$ for a morphism $\xi: \Sigma(K) \rightarrow E$. Then $\mathrm{H}_{T}(\sigma \circ \zeta)=\mathrm{H}_{T}(\kappa \circ \xi)=0$, since $\mathrm{H}_{T}(\kappa)=0$. Therefore $\mathrm{H}_{T}(\sigma \circ \zeta)=0$ and consequently $\sigma \circ \zeta=0$. It follows that $\zeta=\alpha \circ \theta$ for some morphism $\theta: T_{0} \rightarrow E$. This implies that the complex $(*)$ is exact and therefore $\operatorname{Ext}_{\Lambda_{T}}^{1}\left[F, \mathrm{H}_{T}(E)\right]=0$.

As a consequence of Lemma 3.8 we have the following.

PROPOSITION 3.9. Let $T$ be an object in $\mathcal{C}$ with local endomorphism ring and assume that $\operatorname{add}(T)$ is contravariantly finite in $\mathcal{C}$. Then the following are equivalent. 
(i) There exists an AR-triangle $\Sigma^{-1} E \rightarrow A \rightarrow T \rightarrow E$ in $\mathcal{C}$ and the left $\Lambda_{T^{-}}$ module $\mathrm{H}_{T}(E)$ is injective.

(ii) The functor $\mathrm{H}_{T, \mathfrak{m}}^{\vee}:=\operatorname{Hom}_{\Lambda_{T}}\left[\mathrm{H}_{T}(-), I_{\mathfrak{m}}\right]: \mathcal{C}^{\mathrm{op}} \rightarrow \mathcal{A} b$ is representable.

Proof. Part (ii) $\Rightarrow$ (i) follows directly from Corollary 3.5 and Lemma 3.6. If (i) holds, then, by Lemma 3.6, the left $\Lambda_{T}$-module $\mathrm{H}_{T}(E)$ is the injective envelope $I_{\mathfrak{m}}$ of the simple $\Lambda_{T}$-module $S_{\mathfrak{m}}$ and by Lemma 3.8 the canonical map $\mathcal{C}(-, E) \rightarrow \operatorname{Hom}_{\Lambda_{T}}\left(\mathrm{H}_{T}(-), \mathrm{H}_{T}(E)\right)$ is invertible. We infer that $I_{\mathfrak{m}}$ is $T$ realizable by the object $E$. Then by Proposition 2.8 we conclude that the functor $\mathrm{H}_{T, \mathrm{~m}}^{\vee}$ is representable.

Now we can prove the following result which, under a finiteness condition, shows that the existence of an AR-triangle starting at an object $T$ is equivalent to the representability of the injective envelope of the simple functor associated to $T$.

THEOREM 3.10. Let $\mathcal{C}$ be an R-linear triangulated category over a commutative Noetherian ring $R$ and let $T$ be an object in $\mathcal{C}$ with local endomorphism ring. If $\forall C \in \mathcal{C}$ the $R$-module $\mathcal{C}(T, C)$ is finitely generated, then the following are equivalent:

(i) There exists an AR-triangle $\Sigma^{-1} E \rightarrow A \rightarrow T \rightarrow E$ in $\mathcal{C}$.

(ii) The functor $\mathrm{H}_{T, \mathrm{~m}}^{\vee}:=\operatorname{Hom}_{\Lambda_{T}}\left[\mathrm{H}_{T}(-), I_{\mathrm{m}}\right]: \mathcal{C}^{\mathrm{op}} \rightarrow \mathcal{A} b$ is representable.

If (i) holds, then we have isomorphisms: $E \cong \mathbb{D}_{\mathfrak{m}}(T)$ and $\mathrm{H}_{T, \mathfrak{m}}^{\vee} \cong \mathcal{C}(-, E)$.

Proof. The implication (ii) $\Rightarrow$ (i) follows by Proposition 3.9.

(i) $\Rightarrow$ (ii) By Lemma 3.7 it follows that $\operatorname{add}(T)$ is contravariantly finite in $\mathcal{C}$. Since $\mathcal{C}(T, T)$ is finitely generated as an $R$-module and $R$ is Noetherian, the local ring $\Lambda_{T}=\mathcal{C}(T, T)$ is Noetherian. Then the FP-injective left $\Lambda_{T}$-module $\mathrm{H}_{T}(E)$ is injective and therefore the functor $\mathrm{H}_{T, \mathrm{~m}}^{\vee}$ is representable by Proposition 3.9.

Summarizing the above results we have the following.

THEOREM 3.11. Let $\mathcal{C}$ be a skeletally small R-linear triangulated KrullSchmidt category over a commutative Noetherian ring and assume that the $R$-module $\mathcal{C}(A, B)$ is finite generated, for all objects $A, B$ in $\mathcal{C}$. Then the following are equivalent:

(i) $\mathcal{C}$ has right AR-triangles.

(ii) The injective envelopes of the simple functors $\mathcal{C}^{\text {op }} \rightarrow \mathcal{A} b$ are representable.

(iii) Any simple contravariant functor $\mathcal{C}^{\mathrm{op}} \rightarrow \mathcal{A} b$ is finitely presented.

(iv) For any object $T$ in $\mathcal{C}$ with local endomorphism ring, the injective envelope of the unique simple $\operatorname{End}_{\mathcal{C}}(T)$-module is T-realizable. 
Proof. By our previous results we have (i) $\Leftrightarrow$ (iv) and (ii) $\Rightarrow$ (i). The implication (i) $\Rightarrow$ (ii) follows from Proposition 2.1, Theorem 3.10 and the fact that, since $\mathcal{C}$ is Krull-Schmidt, any simple functor $\mathcal{C}^{\mathrm{op}} \rightarrow \mathcal{A} b$ is of the form $S_{T, \mathfrak{m}}$ where $T$ has local endomorphism ring, see [2, Proposition 2.3]. If (ii) holds, then $S_{T, m}$ is finitely presented as the image of a morphism between representable functors. Finally if (iii) holds, then since $\mathcal{C}$ is Krull-Schmidt and triangulated it follows from $[28,29]$ that the category mod- $\mathcal{C}$ is an abelian Frobenius category with injective envelopes which are therefore representable. This clearly implies (ii).

Remark 3.12. For any additive functor $F: \mathcal{C} \rightarrow \mathcal{A} b$, the abelian group $F(T)$ carries a natural right $\Lambda_{T}$-module structure by defining: $x \star \rho:=$ $F(\rho)(x), \forall x \in F(T)$ and $\forall \rho \in \Lambda_{T}$. In particular we have the (co)homological functors

$$
\begin{gathered}
\mathrm{H}^{T}: \mathcal{C}^{\text {op }} \rightarrow \operatorname{Mod}-\Lambda_{T}, \mathrm{H}^{T}(A)=\mathcal{C}(A, T) \text { and } \\
\mathrm{H}_{\wedge}^{T, \mathfrak{n}}:=\operatorname{Hom}_{\Lambda_{T}}\left[\mathrm{H}^{T}(-), I^{\mathfrak{n}}\right]: \mathcal{C} \rightarrow \mathcal{A} b
\end{gathered}
$$

where $\mathrm{n}$ is a maximal right ideal of $\Lambda_{T}$ and $I^{\mathrm{n}}$ is the injective envelope of the simple right $\Lambda_{T}$-module $\Lambda_{T} / \mathfrak{r}$. Using the functors $\mathrm{H}^{T}$ and $\mathrm{H}_{\wedge}^{T, \mathfrak{n}}$, all the results of this and the previous section have their dual versions. We shall use freely the dual versions leaving their proof to the reader. For later use we only mention the following.

THEOREM 3.13. Let $\mathcal{C}$ be an R-linear triangulated category over a commutative Noetherian ring $R$. Assume that for all objects $A, C$ in $\mathcal{C}$, the $R$-module $\mathcal{C}(A, C)$ is finitely generated. Then the following are equivalent.

(i) $\mathcal{C}$ has AR-triangles.

(ii) The functors $\mathrm{H}_{\wedge}^{T, \mathfrak{n}}$ and $\mathrm{H}_{T, \mathrm{~m}}^{\vee}$ are representable, in which case we have:

$$
\mathrm{H}_{\wedge}^{T, \mathfrak{n}} \cong \mathcal{C}\left(\Sigma^{-1} \tau^{-}(T),-\right) \text { and } \mathrm{H}_{T, \mathrm{~m}}^{\vee} \cong \mathcal{C}\left(-, \Sigma \tau^{+}(T)\right) .
$$

\section{Auslander-Reiten Triangles, Dualizing Categories and Serre Functors}

Our aim in this section is to study when a triangulated category admits globally AR-triangles. We are also interested in having an internal description of the Auslander-Reiten translations. Working in the appropriate setting, this is related to the existence of Serre functors and a certain dualizing property of the category.

\subsection{AR-TRIANGLES IN $R$-FINITE CATEGORIES}

Throughout this section $\mathcal{C}$ denotes a skeletally small $R$-linear triangulated category with split idempotents over a commutative ring $R$. There are several 
finiteness conditions one can impose on $\mathcal{C}$. We are especially interested in case $\mathcal{C}$ is finite over $R$ in the following sense.

DEFINITION 4.1. We say that $\mathcal{C}$ is $R$-finite if one of the following conditions hold:

(I) $R$ is Noetherian and the $R$-module $\mathcal{C}(A, B)$ is of finite length, $\forall A, B \in \mathcal{C}$.

(II) $R$ is Artinian and the $R$-module $\mathcal{C}(A, B)$ is finitely generated, $\forall A, B \in \mathcal{C}$.

(III) $R$ is Noetherian complete local and the $R$-module $\mathcal{C}(A, B)$ is finitely generated, $\forall A, B \in \mathcal{C}$.

Let $I$ be the minimal injective cogenerator of Mod- $R$ and let $\mathrm{D}$ be the functor $\operatorname{Hom}_{R}(-, I):(\operatorname{Mod}-R)^{\text {op }} \rightarrow$ Mod- $R$. We denote by noeth $(R)=\bmod -R$, resp. $\operatorname{art}(R)$, the category of Noetherian, resp. Artinian, $R$-modules, and let $\operatorname{fin}(R)=\operatorname{noeth}(R) \cap \operatorname{art}(R)$ be the category of finite length modules. It is wellknown that the functor D induces a duality $\mathrm{D}: \operatorname{fin}(R) \rightarrow \operatorname{fin}(R)$ in cases (I) and (II), and a duality D : noeth $(R) \rightarrow \operatorname{art}(R)$ in case (III), see [62] and Sections I.4 and I.5 of the more comprehensive [4] for details.

From now on we assume that the $R$-linear triangulated category $\mathcal{C}$ is $R$-finite.

If $T$ is an object in $\mathcal{C}$, then the endomorphism $\operatorname{ring} \Lambda_{T}$ of $T$ is a Noetherian $R$-algebra (Artinian in case (II)) and $\mathrm{D}\left(\Lambda_{T}\right)$ is an injective cogenerator of Mod- $\Lambda_{T}$. We denote by $D$ the functor $\operatorname{Hom}_{R}(-, I):\left(\operatorname{Mod}-\Lambda_{T}\right)^{\mathrm{op}} \rightarrow \operatorname{Mod}-\Lambda_{T}^{\mathrm{op}}$. Note that $\operatorname{Hom}_{R}(-, I) \stackrel{\cong}{\rightarrow} \operatorname{Hom}_{\Lambda_{T}}\left(-, \operatorname{Hom}_{R}(\Lambda, I)\right)=\operatorname{Hom}_{\Lambda_{T}}\left(-, \mathrm{D}\left(\Lambda_{T}\right)\right)$ as functors : $\left(\operatorname{Mod}-\Lambda_{T}\right)^{\text {op }} \rightarrow \operatorname{Mod}-\Lambda_{T}^{\text {op }}$. Recall from Section 2 that for an object $T$ in $\mathcal{C}$ with local endomorphism ring $\Lambda_{T}$ and maximal ideal $\mathrm{m}$, the functor $\mathrm{H}_{T, \mathfrak{m}}^{\vee}=\operatorname{Hom}_{\Lambda_{T}}\left[\mathcal{C}(T,-), I_{\mathfrak{m}}\right]$, resp. $\mathrm{H}_{\wedge}^{T, \mathfrak{m}}=\operatorname{Hom}_{\Lambda_{T}}\left[\mathcal{C}(-, T), I^{\mathfrak{m}}\right]$, is the injective envelope of the simple contravariant, resp. covariant, functor determined by $T$ and $\mathrm{m}$.

LEMMA 4.2. The category $\mathcal{C}$ is Krull-Schmidt and for any object $X \in \mathcal{C}$, the subcategory $\operatorname{add}(X)$ is functorially finite in $\mathcal{C}$. Moreover for any indecomposable object $T \in \mathcal{C}$, there exist isomorphisms of functors:

$$
\mathrm{H}_{T, \mathrm{~m}}^{\vee} \cong \mathrm{DC}(T,-) \text { and } \mathrm{H}_{\wedge}^{T, \mathrm{~m}} \cong \mathrm{DC}(-, T) \text {. }
$$

Proof. Since the endomorphism ring of any object of $\mathcal{C}$ is a Noetherian $R$-algebra, by [4, Section I.5], it follows that $\mathcal{C}$ is Krull-Schmidt, and functorial finiteness of $\operatorname{add}(T)$ follows from Lemma 3.7. Since $\mathcal{C}$ is $R$-finite and the $R$-module $\Lambda_{T}=\operatorname{Hom}_{\mathcal{C}}(T, T)$ is finitely generated, it follows that the natural map $\Lambda_{T} \rightarrow \mathrm{D}^{2}\left(\Lambda_{T}\right)$ is invertible. Consequently the injective cogenerator $\mathrm{D}\left(\Lambda_{T}\right)$ of $\Lambda_{T}$ is indecomposable. Then clearly $\mathrm{D}\left(\Lambda_{T}\right)$ is isomorphic to the minimal injective cogenerator $I_{\mathfrak{m}}$ and the assertion follows from the isomorphisms (the proof that $\mathrm{H}_{\wedge}^{T, \mathfrak{m}} \cong \mathrm{DC}(-, T)$ is dual): 


$$
\begin{aligned}
& \mathrm{H}_{T, \mathfrak{m}}^{\vee}=\operatorname{Hom}_{\Lambda_{T}}\left[\mathcal{C}(T,-), I_{\mathfrak{m}}\right] \cong \operatorname{Hom}_{\Lambda_{T}}\left[\mathcal{C}(T,-), \mathrm{D}\left(\Lambda_{T}\right)\right] \cong \\
& \left.\operatorname{Hom}_{\Lambda_{T}}\left[\mathcal{C}(T,-), \operatorname{Hom}_{R}\left(\Lambda_{T}, I\right)\right] \cong \operatorname{Hom}_{R}\left[\Lambda_{T} \otimes_{\Lambda_{T}} \mathcal{C}(T,-), I\right)\right] \cong \\
& \left.\operatorname{Hom}_{R}[\mathcal{C}(T,-), I)\right] \cong \mathrm{DC}(T,-) .
\end{aligned}
$$

COROLLARY 4.3. Let $\mathcal{C}$ be a triangulated $R$-finite category over a commutative ring $R$. Then $\mathcal{C}$ has AR-triangles iff for any indecomposable object $X \in \mathcal{C}$, the functors $\mathrm{DC}(X,-)$ and $\mathrm{DC}(-, X)$ are representable. In this case we have isomorphisms:

$$
\mathcal{C}\left(-, \Sigma \tau^{+}(X)\right) \cong \mathrm{DC}(X,-), \mathcal{C}\left(\Sigma^{-1} \tau^{-}(X),-\right) \cong \mathrm{DC}(-, X) .
$$

\subsection{DERIVED CATEGORIES}

If $\mathcal{A}$ is an abelian category, we denote by $\mathbf{D}(\mathcal{A})$, resp. $\mathbf{D}^{\mathrm{b}}(\mathcal{A})$, the unbounded, resp. bounded, derived category of $\mathcal{A}$ in the sense of Verdier [77]. Now let $\Lambda$ be an $R$-algebra over a commutative ring $R$. As before we denote by $\mathrm{D}$ the functor $\operatorname{Hom}_{R}(-, I):(\operatorname{Mod}-R)^{\mathrm{op}} \rightarrow \operatorname{Mod}-R$, where $I$ is the minimal injective cogenerator of Mod- $R$. Similarly we denote by $\mathrm{D}$ any one of the total derived functors $\mathbb{R} \operatorname{Hom}_{R}(-, I): \mathbf{D}(\operatorname{Mod}-R)^{\mathrm{op}} \rightarrow \mathbf{D}(\operatorname{Mod}-R)$ and $\mathbb{R} \operatorname{Hom}_{R}(-, \mathrm{D}(\Lambda))$ : $\mathbf{D}(\operatorname{Mod}-\Lambda)^{\text {op }} \rightarrow \mathbf{D}\left(\operatorname{Mod}-\Lambda^{\mathrm{op}}\right)$. Notice that $\mathbb{R} \operatorname{Hom}_{R}(-, I) \stackrel{\cong}{\rightarrow} \mathbb{R} \operatorname{Hom}_{\Lambda}(-, \mathrm{D}(\Lambda))$ as triangulated functors $\mathbf{D}(\operatorname{Mod}-\Lambda)^{\text {op }} \rightarrow \mathbf{D}\left(\operatorname{Mod}-\Lambda^{\text {op }}\right)$. We denote by $\mathcal{P}_{\Lambda}$ the category of finitely generated projective $\Lambda$-modules and by $\mathcal{H}^{\mathrm{b}}\left(\mathcal{P}_{\Lambda}\right)$ the bounded homotopy category of $\mathcal{P}_{\Lambda}$. Recall that a complex $X$ of $\Lambda$-modules is called perfect if $X$ is quasi-isomorphic to a complex in $\mathcal{H}^{\mathrm{b}}\left(\mathcal{P}_{\Lambda}\right)$. Fix a perfect complex $X$ in $\mathbf{D}(\operatorname{Mod}-\Lambda)$. Then for any complex $Y$ in $\mathbf{D}(\operatorname{Mod}-\Lambda)$, there exist natural isomorphisms in $\mathbf{D}(\operatorname{Mod}-\Lambda)$ :

$$
\mathbb{R H o m}_{\Lambda}(X, Y) \stackrel{\cong}{\rightarrow} Y \otimes_{\Lambda}^{\mathbb{L}} \mathbb{R} \operatorname{Hom}_{\Lambda}(X, \Lambda), \quad \operatorname{DRH}_{\Lambda}(X, \Lambda) \stackrel{\cong}{\rightarrow} X \otimes_{\Lambda}^{\mathbb{L}} \mathrm{D}(\Lambda)
$$

induced by the well-known isomorphisms $\operatorname{Hom}_{\Lambda}(P, A) \stackrel{\cong}{\rightrightarrows} A \otimes_{\Lambda} \operatorname{Hom}_{\Lambda}(P, \Lambda)$ and $\operatorname{DHom}_{\Lambda}(P, \Lambda) \stackrel{\cong}{\rightarrow} P \otimes_{\Lambda} \mathrm{D}(\Lambda)$, where $P \in \mathcal{P}_{\Lambda}$ and $A \in$ Mod- $\Lambda$. Applying $\mathrm{D}$ to the first one and using the second we have isomorphisms:

$$
\begin{aligned}
& \mathrm{DRHom}_{\Lambda}(X, Y) \stackrel{\cong}{\rightarrow} \mathbb{R} \operatorname{Hom}_{\Lambda}\left[Y \otimes_{\Lambda}^{\mathbb{L}} \mathbb{R} \operatorname{Hom}_{\Lambda}(X, \Lambda), \mathrm{D}(\Lambda)\right] \stackrel{\cong}{\rightrightarrows} \\
& \mathbb{R H o m}_{\Lambda}\left[Y, \mathbb{R H o m}_{\Lambda}\left(\mathbb{R} \operatorname{Hom}_{\Lambda}(X, \Lambda), \mathrm{D}(\Lambda)\right)\right] \stackrel{\cong}{\rightarrow} \mathbb{R} \operatorname{Hom}_{\Lambda}\left[Y, \operatorname{DRHom}_{\Lambda}(X, \Lambda)\right] \\
& \stackrel{\cong}{\rightarrow} \operatorname{Hom}_{\Lambda}\left(Y, X \otimes_{\Lambda}^{\mathbb{L}} \mathrm{D}(\Lambda)\right)
\end{aligned}
$$

Applying $\mathrm{H}^{0}$ we get an isomorphism

$$
\operatorname{DHom}_{\Lambda}(X, Y) \stackrel{\cong}{\rightarrow} \operatorname{Hom}_{\Lambda}\left(Y, X \otimes_{\Lambda}^{\mathbb{L}} \mathrm{D}(\Lambda)\right) .
$$

The following observation gives an explicit description of the dual object $\mathbb{D}_{\mathfrak{m}}(X)$. 
LEMMA 4.4. Let $X$ be a perfect complex with local endomorphism ring. If the $R$-module $\operatorname{Hom}_{\Lambda}(X, X)$ is finitely generated, then the following are equivalent.

(i) There exists an isomorphism $\mathbb{D}_{\mathfrak{m}}(X) \stackrel{\cong}{\rightrightarrows} X \otimes_{\Lambda}^{\mathbb{L}} \mathrm{D}(\Lambda)$ in $\mathbf{D}$ (Mod- $\left.\Lambda\right)$.

(ii) The $\Lambda_{X}$-module $\mathrm{D}\left(\Lambda_{X}\right)$ is indecomposable.

Proof. If $\mathrm{D}\left(\Lambda_{X}\right)$ is indecomposable, then using the isomorphism $(\dagger)$ and working as in Lemma 4.2 we obtain that $\mathrm{D}\left(\Lambda_{X}\right)$ is isomorphic to $I_{\mathrm{m}}$ and the functors $\mathrm{H}_{X, \mathrm{~m}}^{\vee}$ and $\operatorname{DHom}_{\Lambda}(X,-)$ are isomorphic. Therefore $\mathrm{H}_{X, \mathfrak{m}}^{\vee}$ is representable in $\mathbf{D}(\operatorname{Mod}-\Lambda)$, in particular the dual object $\mathbb{D}_{\mathfrak{m}}(X)$ exists in D(Mod- $\Lambda)$, and we have isomorphisms: $\mathrm{H}_{X, \mathrm{~m}}^{\vee} \stackrel{\cong}{\rightarrow} \operatorname{Hom}_{\Lambda}\left[-, X \otimes_{\Lambda}^{\mathbb{L}} \mathrm{D}(\Lambda)\right]$ and $\mathbb{D}_{\mathfrak{m}}(X) \stackrel{\cong}{\rightarrow} X \otimes_{\Lambda}^{\mathbb{L}} \mathrm{D}(\Lambda)$. Conversely if (i) holds, then the isomorphism $(\dagger)$ shows that $\mathrm{D}\left(\Lambda_{X}\right) \cong I_{\mathfrak{m}}$ which is indecomposable.

It is easy to see that condition (ii) above holds, if the bounded homotopy category $\mathcal{H}^{\mathrm{b}}\left(\mathcal{P}_{\Lambda}\right)$, which is isomorphic in $\mathbf{D}(\operatorname{Mod}-\Lambda)$ to the subcategory of perfect complexes, is $R$-finite, for instance if $\Lambda$ is an Artin $R$-algebra or a Noetherian $R$-algebra over a commutative Noetherian complete local ring $R$. Hence from Lemma 4.4 we deduce the following consequence (the complete case is due to Krause [57]), referring to section 9 for a further discussion of Auslander-Reiten theory in derived categories.

COROLLARY 4.5. Let $\Lambda$ be a Noetherian R-algebra over a commutative Noetherian ring $R$, and assume that either $\Lambda$ is Artinian or else $R$ is complete and local. Then for any indecomposable perfect complex $X$ in $\mathbf{D}(\operatorname{Mod}-\Lambda)$, there exists an AR-triangle

$$
X \otimes_{\Lambda}^{\mathbb{L}} \mathrm{D}(\Lambda)[-1] \longrightarrow A \longrightarrow X \longrightarrow X \otimes_{\Lambda}^{\mathbb{L}} \mathrm{D}(\Lambda)
$$

in $\mathbf{D}$ (Mod- $\Lambda)$. Moreover $\tau^{+}(X)=X \otimes_{\Lambda}^{\mathbb{L}} \mathrm{D}(\Lambda)[-1]$ lies in the full subcategory $\mathbf{D}_{\mathrm{art}}^{\mathrm{b}}(\operatorname{Mod}-\Lambda)$ of $\mathbf{D}^{\mathrm{b}}(\operatorname{Mod}-\Lambda)$ consisting of all complexes with Artinian cohomology.

\subsection{DUALIZING CATEGORIES AND SERRE FUNCTORS}

If $\mathcal{U}$ is one of the subcategories noeth $(R), \operatorname{art}(R)$ or $\operatorname{fin}(R)$ of $\operatorname{Mod}-R$, we denote by $\left[\mathcal{C}^{\mathrm{op}}, \mathcal{U}\right]$, resp. $[\mathcal{C}, \mathcal{U}]$, the category of contravariant, resp. covariant, additive functors $\mathcal{C} \rightarrow \mathcal{U}$. Then clearly the duality $\mathrm{D}$ induces a duality $\mathrm{D}:\left[\mathcal{C}^{\mathrm{op}}\right.$, fin $\left.(R)\right] \rightarrow[\mathcal{C}$, fin $(R)]$ in cases (I) and (II), and a duality $\mathrm{D}:\left[\mathcal{C}^{\mathrm{op}}\right.$, $\operatorname{noeth}(R)] \rightarrow[\mathcal{C}, \operatorname{art}(R)]$ in case (III).

It is easy to see that the functors $\operatorname{DC}(X,-)$, for $X$ indecomposable in $\mathcal{C}$, form a cogenerating set $\mathcal{R}$ of indecomposable injectives in Mod-C. We say that an additive functor $F: \mathcal{C}^{\mathrm{op}} \rightarrow \mathcal{A} b$ is finitely copresented if $F$ admits a 
copresentation $0 \rightarrow F \rightarrow J_{0} \rightarrow J_{1}$ where the $J_{i}$ are in $\operatorname{add}(\mathcal{R})$. Equivalently there exists an exact sequence $0 \rightarrow F \rightarrow \mathrm{DC}(X,-) \rightarrow \mathrm{DC}(Y,-)$. We denote by mod- ${ }^{\vee} \mathcal{C}$ the full subcategory of Mod- $\mathcal{C}$ consisting of finitely copresented functors. The category mod- ${ }^{\vee} \mathcal{C}^{\text {op }}$ is defined similarly. It is easy to see that mod- $\mathcal{C}$, mod- $^{\vee} \mathcal{C} \subseteq\left[\mathcal{C}^{\mathrm{op}}, \operatorname{fin}(R)\right]$ and $\bmod -\mathcal{C}^{\mathrm{op}}$, $\bmod ^{\vee}{ }^{\vee} \mathcal{C}^{\mathrm{op}} \subseteq[\mathcal{C}$, fin $(R)]$ in cases (I) and (II), and mod-C $\subseteq\left[\mathcal{C}^{\text {op }}, \operatorname{noeth}(R)\right]$, mod- $\mathcal{C}^{\text {op }} \subseteq[\mathcal{C}$, noeth $(R)]$, and mod- ${ }^{\vee} \mathcal{C} \subseteq\left[\mathcal{C}^{\text {op }}, \operatorname{art}(R)\right]$, mod- $^{\vee} \mathcal{C}^{\text {op }} \subseteq[\mathcal{C}$, art $(R)]$ in case (III). We are especially interested in case the full subcategory of finitely copresented functors coincides with the full subcategory of finitely presented ones. We have the following characterizations which follow easily from the definitions.

LEMMA 4.6. The following are equivalent.

(i) $\bmod ^{\vee} \mathcal{C} \subseteq \bmod -\mathcal{C}$ and $\bmod ^{\vee}{ }^{\vee} \mathcal{C}^{\text {op }} \subseteq \bmod -\mathcal{C}^{\text {op }}$.

(ii) $\bmod ^{\vee} \mathcal{C}=$ mod- $\mathcal{C}$.

(iii) mod- ${ }^{\vee} \mathcal{C}^{\text {op }}=$ mod- $\mathcal{C}^{\text {op }}$.

(iv) The duality $\mathrm{D}$ induces a duality $\mathrm{D}: \bmod -\mathcal{C} \rightarrow \bmod -\mathcal{C}^{\mathrm{op}}$.

If (i) holds, then the $R$-module $\mathcal{C}(A, B)$ has finite length for all objects $A, B$ in $\mathcal{C}$.

The above result suggests the following definition which extends slightly the notion of a dualizing variety introduced by Auslander-Reiten [6] in connection with stable equivalence and existence of Auslander-Reiten sequences for Artin algebras.

DEFINITION 4.7. An $R$-linear triangulated category $\mathcal{C}$ over a commutative ring $R$ is called dualizing if $\mathcal{C}$ is $R$-finite and satisfies the conditions of Lemma 4.6.

Recall from [23] or [75] that an additive functor $S: \mathcal{C} \rightarrow \mathcal{C}$ is a right Serre functor for $\mathcal{C}$ provided that there exists a natural isomorphism of bifunctors $\mathrm{DC}(A, B) \stackrel{\cong}{\rightrightarrows} \mathcal{C}(B, \mathrm{~S}(A))$. Left Serre functors are defined dually, and a Serre functor is a left and right Serre functor. It is known that a Serre functor is uniquely determined up to isomorphism and it is a triangulated equivalence, see [23, Proposition 3.3], [75, Lemma I.1.5]. The following result generalizes results of Reiten and Van den Bergh [75, Proposition I.2.3].

PROPOSITION 4.8. Let $\mathcal{C}$ be a triangulated $R$-finite category over a commutative ring $R$. Then the following are equivalent.

(i) $\mathcal{C}$ has right, resp. left, AR-triangles.

(ii) $\mathcal{C}$ admits a right, resp. left, Serre functor.

(iii) $\bmod ^{\vee} \mathcal{C} \subseteq \bmod -\mathcal{C}$, resp. $\bmod ^{\vee} \mathcal{C}^{\text {op }} \subseteq \bmod -\mathcal{C}^{\text {op }}$.

Proof. By Lemma 4.2, $\mathcal{C}$ is Krull-Schmidt and $\mathrm{H}_{T, \mathrm{~m}}^{\vee} \stackrel{\cong}{\cong} \mathcal{C}(T,-)$ for any indecomposable object $T$ in $\mathcal{C}$. Hence $\mathcal{C}$ has right AR-triangles iff $\mathrm{DC}(T,-)$ is representable. Clearly this is equivalent to say that $\mathcal{C}$ has a right Serre 
functor. Assume now that $S: \mathcal{C} \rightarrow \mathcal{C}$ is a right Serre functor for $\mathcal{C}$. Then the isomorphism $\mathrm{DC}(X,-) \rightarrow \mathcal{C}(-, \mathrm{S}(X))$ shows that any finitely copresented contravariant functor is finitely presented, i.e. $\bmod ^{\vee} \mathcal{C} \subseteq \bmod -\mathcal{C}$. If this holds, then for any indecomposable object $T$ of $\mathcal{C}$, the finitely copresented functor $\mathrm{DC}(T,-)$ is finitely presented. Since $\mathrm{DC}(T,-)$ is an indecomposable injective functor in mod-C and the latter is Frobenius, it follows that $\mathrm{DC}(T,-) \cong \mathrm{H}_{T, \mathrm{~m}}^{\vee}$ is representable. This implies that $\mathcal{C}$ has right AR-triangles by Theorem 3.11. The case of left AR-triangles, left Serre functors and finitely copresented covariant functors is similar. I.2.4].

We have the following nice consequence which generalizes [75, Theorem

THEOREM 4.9. Let $\mathcal{C}$ be a triangulated $R$-finite category over a commutative ring $R$. Then the following are equivalent.

(i) $\mathcal{C}$ has AR-triangles.

(ii) $\mathcal{C}$ admits a Serre functor $\mathrm{S}$.

(iii) $\mathcal{C}$ is dualizing.

If (i) holds, then for any indecomposable object $T \in \mathcal{C}$ we have isomorphisms:

$$
\begin{aligned}
& \mathrm{H}_{T, \mathfrak{m}}^{\vee} \cong \mathrm{DC}(T,-) \cong \mathcal{C}(-, \mathrm{S}(T)) \cong \mathcal{C}\left(-, \Sigma \tau^{+}(T)\right), \\
& \mathrm{H}_{\wedge}^{T, \mathrm{~m}} \cong \mathrm{DC}(-, T) \cong \mathcal{C}\left(\mathrm{S}^{-1}(T),-\right) \cong \mathcal{C}\left(\Sigma^{-1} \tau^{-}(T),-\right) .
\end{aligned}
$$

Let $\mathcal{C}$ be a triangulated $R$-finite category over a commutative ring $R$. For any object $X$ in $\mathcal{C}$, the support of the functor $\mathcal{C}(-, X)$, resp. $\mathcal{C}(X,-)$, is the full subcategory of $\mathcal{C}$ consisting of all objects $Y$ such that $\mathcal{C}(Y, X) \neq 0$, resp. $\mathcal{C}(X, Y) \neq 0$, and is denoted by $\operatorname{Supp} \mathcal{C}(-, X)$, resp. $\operatorname{Supp} \mathcal{C}(X,-)$. Examples of triangulated categories $\mathcal{C}$ satisfying the property that $\operatorname{Supp} \mathcal{C}(-, X)$ or $\operatorname{SuppC}(X,-)$ contains only finitely many indecomposable objects, include stable categories modulo projectives of the form $\bmod -\mathcal{T}^{\mathbb{Z}}$, where $\mathcal{T}^{\mathbb{Z}}$ is the repetition of a locally bounded category $\mathcal{T}$ over a field, see [31, Section 8.3]. By a result of Reiten [74, Proposition 7.1.5], such categories are dualizing. Therefore we have the following consequence which gives an alternative proof to a recent result of Xiao and Zhu [79, Proposition 1.3].

COROLLARY 4.10. If for any object $X$ in $\mathcal{C}$, one of the subcategories $\operatorname{SuppC}(-, X)$ or $\operatorname{SuppC}(X,-)$ contains only finitely many indecomposable objects, then $\mathcal{C}$ has AR-triangles and admits a Serre functor.

There are examples of triangulated categories for which some positive power of the suspension $\Sigma$ is a Serre functor.

EXAMPLE 4.11. Let $\mathbb{X}$ be a connected compact complex analytic manifold, resp. a non-singular projective variety. Then the derived category $\mathbf{D}_{\mathrm{coh}}^{\mathrm{b}}(\mathbb{X})$, resp. $\mathbf{D}^{\mathrm{b}}(\operatorname{coh} \mathbb{X})$, of, resp. coherent, sheaves of $\mathcal{O}_{\mathbb{X}}$-modules with coherent 
cohomology admits the Serre functor $-\otimes_{\mathcal{O}_{\mathbb{X}}} \omega_{\mathbb{X}}[\operatorname{dim} \mathbb{X}]$, see [24, Section 5]. Hence $\mathbf{D}_{\text {coh }}^{\mathrm{b}}(\mathbb{X})$ or $\mathbf{D}^{\mathrm{b}}(\operatorname{coh} \mathbb{X})$ has AR-triangles and consequently $\mathbb{X}$ is CalabiYau iff $-[\operatorname{dim} \mathbb{X}]$ is a Serre functor.

\subsection{STRONG GENERATORS}

Throughout this subsection we fix a triangulated $R$-linear category $\mathcal{C}$ with split idempotents over a commutative Noetherian ring $R$. If $M$ is an $R$-module, then we denote by $\operatorname{dim}_{R} M$ its composition length. Following Reiten and Van den Bergh [75] we say that $\mathcal{C}$ is Ext-finite if $\sum_{n \in \mathbb{Z}} \operatorname{dim}_{R} \mathcal{C}\left(A, \Sigma^{n}(B)\right)<\infty$ for all $A, B$ in $\mathcal{C}$. Recall that a cohomological functor $F: \mathcal{C}^{\text {op }} \rightarrow$ Mod- $R$ is said to be of finite type provided that $\sum_{n \in \mathbb{Z}} \operatorname{dim}_{R} F \Sigma^{n}(A)<\infty$, for all $A \in \mathcal{C}$. We refer to Reiten and Van den Bergh [75] for a classification of hereditary Noetherian abelian Ext-finite $k$-categories $\mathcal{A}$ over a field $k$ such that the bounded derived category $\mathbf{D}^{\mathrm{b}}(\mathcal{A})$ admits a Serre functor (and therefore AR-triangles). Following Bondal and Van den Bergh [24], we say that an object $X$ in $\mathcal{C}$ is a strong generator of $\mathcal{C}$ provided that there exists $n \geqslant 0$ such that any object of $\mathcal{C}$ can be obtained from $X$ by taking finite direct sums, direct summands, shifts and at most $n-1$ cones.

THEOREM 4.12. Let $\mathcal{C}$ be a skeletally small Ext-finite R-linear triangulated category over a commutative Noetherian ring $R$. If $\mathcal{C}$ admits a strong generator, then $\mathcal{C}$ has AR-triangles, is dualizing and admits a Serre functor.

Proof. Clearly $\mathcal{C}$ is $R$-finite. Therefore for any object $T$ in $\mathcal{C}$ with local endomorphism ring, we have an isomorphism of functors $\mathrm{H}_{T, \mathfrak{m}}^{\vee} \cong \mathrm{DC}(T,-)$ and the functor $\mathrm{DC}(T,-)$ is of finite type. Since $\mathcal{C}$ admits a strong generator, by a result of Bondal and Van den Bergh, see [24, Theorem 1.3], it follows that the functor $\mathrm{DC}(T,-)$, hence $\mathrm{H}_{T, \mathfrak{m}}^{\vee}$, is representable. Then Corollary 3.5 implies that $\mathcal{C}$ has right AR-triangles. Dually we have an isomorphism of functors $\mathrm{H}_{\wedge}^{T, \mathfrak{m}} \cong \mathrm{DC}(-, T)$ and $\mathrm{DC}(-, T)$ is of finite type. Clearly if $X$ is a strong generator for $\mathcal{C}$, then $X$ is a strong generator for $\mathcal{C}^{\text {op }}$. Then as above, $\mathrm{DC}(-, T)$, hence $\mathrm{H}_{\wedge}^{T, \mathrm{~m}}$, is representable and then by the dual of Corollary 3.5 we infer that $\mathcal{C}^{\mathrm{op}}$ admits right $\mathrm{AR}$-triangles, or equivalently $\mathcal{C}$ admits left ARtriangles. The remaining assertions follow from Theorem 4.9.

COROLLARY 4.13. Let $\mathbb{X}$ be a smooth scheme over a field $k$. Then the bounded derived category $\mathbf{D}^{\mathrm{b}}(\mathrm{coh} \mathbb{X})$ of coherent sheaves on $\mathbb{X}$ has AR-triangles, is dualizing and admits a Serre functor.

Proof. By [24, Theorem 3.1.4], the category $\mathbf{D}^{b}(\operatorname{coh} \mathbb{X})$ is Ext-finite, has split idempotents and a strong generator. So the assertion follows from Theorem 4.12. 
EXAMPLE 4.14. Let $\Lambda$ be an Artin algebra. We call a strong generator $T$ of $\mathbf{D}^{\mathrm{b}}(\bmod -\Lambda)$ perfect, if $T$ is a perfect complex. Then the following are equivalent.

(i) gl.dim $\Lambda<\infty$.

(ii) $\mathbf{D}^{\mathrm{b}}(\bmod -\Lambda)$ admits a strong perfect generator.

Indeed let gl. $\operatorname{dim} \Lambda=n<\infty$ and consider the proper class of triangles $\mathcal{E}(\Lambda)$ in $\mathbf{D}^{\mathrm{b}}(\bmod -\Lambda)$ generated be $\Lambda$ in the sense of [18]. By [18, Proposition 12.34] the relative global dimension of $\mathbf{D}^{\mathrm{b}}(\bmod -\Lambda)$ with respect to $\mathcal{E}(\Lambda)$ is equal to gl.dim $\Lambda$ which is finite. Then by [18, Corollary 5.5] it follows that $\Lambda$ is a strong generator of $\mathbf{D}^{\mathrm{b}}(\bmod -\Lambda)$. Conversely if $T$ is a strong perfect generator of $\mathbf{D}^{\mathrm{b}}(\bmod -\Lambda)$, then clearly gl. $\operatorname{dim} \Lambda<\infty$. By Theorem 4.12 we obtain as a consequence the well-known result of Happel [35] that if gl. $\operatorname{dim} \Lambda<\infty$, then $\mathbf{D}^{\mathrm{b}}(\bmod -\Lambda)$ has AR-triangles.

One can generalize this example as follows. Let $\mathcal{A}$ be an $R$-linear Artinian abelian category with enough projectives over a commutative Artin ring $R$. If $\mathcal{A}$ has finite global dimension and the set of isoclasses of simple objects of $\mathcal{A}$ is finite, then $\mathbf{D}^{\mathrm{b}}(\mathcal{A})$ has strong generator and therefore has AR-triangles and admits a Serre functor.

Theorem 4.12 can be generalized further as follows. Let $\mathcal{E}$ be a proper class of triangles in $\mathcal{C}$ in the sense of [18]. We refer to [18] for more details on the relative homological algebra in $\mathcal{C}$ based on $\mathcal{E}$; in particular we denote by $\mathcal{E}$-gl. $\operatorname{dim} \mathcal{C}$ the relative global dimension of $\mathcal{C}$ with respect to $\mathcal{E}$. The following result gives a generalization of Theorem 4.12. The latter follows if we consider the proper class in $\mathcal{C}$ induced, in the sense of [18, Example 2.3], by a strong generator.

THEOREM 4.15. Let $\mathcal{C}$ be a skeletally small Ext-finite R-linear triangulated category with split idempotents over a commutative Noetherian ring $R$ which is equipped with a proper class of triangles $\mathcal{E}$. If $\mathcal{E}$-gl. $\operatorname{dim} \mathcal{C}<\infty$, then $\mathcal{C}$ has $A R$ triangles, is dualizing and admits a Serre functor.

Proof. As in Theorem 4.12, $\mathcal{C}$ is $R$-finite and the functor $\mathrm{H}_{T, \mathrm{~m}}^{\vee} \cong \mathrm{DC}(T,-)$ is of finite type. Since $\mathcal{E}$-gl. $\operatorname{dim} \mathcal{C}:=n<\infty$, by [18, Corollary 5.2,] it follows that any object of $\mathcal{C}$ is obtained from $\mathcal{P}(\mathcal{E})$, the category of relative $\mathcal{E}$-projectives, by taking finite direct sums, direct summands, shifts and at most $n-1$ cones. By (a slight generalization of) [24, Lemma 2.4.2] it follows that $\mathrm{H}_{T, \mathfrak{m}}^{\vee}$ is representable. Similarly $\mathrm{H}_{\wedge}^{T, \mathfrak{m}}$ is representable. Therefore $\mathcal{C}$ has ARtriangles by Theorem 3.13.

We close this section with an application of the above results to the derived category of a class of rings which are of interest in non-commutative geometry. Let $\Lambda=\oplus_{n \geq 0} R_{n}$ be a connected graded ring over a field $k=\Lambda_{0}$ and assume throughout that $\operatorname{dim}_{k} \operatorname{Ext}_{\Lambda}^{t}(k, k)<\infty, \forall t \geq 0$. Let Gr Mod- $\Lambda$ be 
the category of left graded $\Lambda$-modules, and let QGr Mod- $\Lambda=\operatorname{Gr} \operatorname{Mod}-\Lambda /$ $\operatorname{Tors}(\Lambda)$ be the quotient in the sense of Gabriel [30], where Tors $(\Lambda)$ is the localizing subcategory of $\mathrm{Gr}$ Mod- $\Lambda$ consisting of all torsion modules, where a graded $\Lambda$-module $M$ is called torsion, if for any $a \in M$, there exists $t \geqslant 0$ such that $\mathrm{m}^{t} a=0$, where $\mathrm{m}=\oplus_{n \geqslant 1} R_{n}$ is the graded maximal ideal of $\Lambda$.

Let $\mathbf{D}(\mathrm{QGr}$ Mod- $\Lambda)$ be the unbounded derived category of the Grothendieck category $\mathrm{QGr} \operatorname{Mod}-\Lambda)$, and let $\mathbf{D}(\mathrm{QGr} \operatorname{Mod}-\Lambda)^{\mathrm{b}}$ be the full subcategory consisting of all compact objects (see the next section for the definition) of $\mathbf{D}(\mathrm{QGr}$ Mod- $\Lambda)$.

THEOREM 4.16. Let $\Lambda$ be a connected graded ring as above satisfying the following:

(i) gl. dim QGr $\operatorname{Mod}-\Lambda<\infty$.

(ii) The functor $\lim \operatorname{Hom}_{\Lambda^{\mathrm{op}}}\left(\Lambda^{\mathrm{op}} / \Lambda_{\geq n}^{\mathrm{op}},-\right)$ has finite cohomological dimension.

(iii) $\forall t \geqslant 0$, the spaces $\stackrel{\lim }{\longrightarrow} \operatorname{Ext}_{\Lambda^{\mathrm{op}}}^{t}\left(\Lambda^{\mathrm{op}} / \Lambda_{\geq n}^{\mathrm{op}}, \Lambda^{\mathrm{op}}\right)$ and $\stackrel{\lim }{\longrightarrow} \operatorname{Ext}_{\Lambda}^{t}\left(\Lambda / \Lambda_{\geq n}, \Lambda\right)$ are finite dimensional and both have right bounded grading.

Then the category $\mathbf{D}(\mathrm{QGr} \text { Mod- } \Lambda)^{\mathrm{b}}$ has AR-triangles.

If $\Lambda$ is left graded coherent, then the bounded derived category $\mathbf{D}^{\mathrm{b}}(\mathrm{Gr} \bmod -\Lambda)$ of the category of finitely presented graded $\Lambda$-modules, has AR-triangles.

Proof. By [24, Theorems 4.2.12, 4.3.4] the imposed assumptions imply that the categories $\mathbf{D}(\mathrm{QGr} \operatorname{Mod}-\Lambda)^{\mathrm{b}}$ and $\mathbf{D}^{\mathrm{b}}(\operatorname{gr} \bmod -\Lambda)$ satisfy the conditions of Theorem 4.12 and the assertion follows.

\section{Categories with Infinite Sums and Torsion Pairs}

In this section we investigate the behavior of Auslander-Reiten triangles with respect to torsion pairs in a triangulated category. Since torsion pairs arise naturally in triangulated categories containing all small coproducts, we study first existence of Auslander-Reiten triangles in this setting where Brown's Representability theorem, when available, provides the main tool for proving representability of the injective envelope of a simple functor.

\subsection{TRIANGULATED CATEGORIES WITH INFINITE SUMS.}

From now on we fix a triangulated category $\mathcal{C}$ which has all small coproducts. For a subcategory $\mathcal{X}$ of $\mathcal{C}$ we denote by $\operatorname{Add}(\mathcal{X})$ the full subcategory of $\mathcal{C}$ consisting of all direct summands of all small coproducts of objects from $\mathcal{X}$. In the sequel we shall need the following infinite analogue of Lemma 3.7, which is a special case of [18, Proposition 8.4,]. First recall that an object $X$ in $\mathcal{C}$ is called compact if the functor 
$\mathcal{C}(X,-): \mathcal{C} \rightarrow \mathcal{A} b$ preserves all small coproducts. We denote by $\mathcal{C}^{\text {b }}$ the full subcategory of $\mathcal{C}$ consisting of the compact objects. It is easy to see that $\mathcal{C}^{\mathrm{b}}$ is a full triangulated subcategory of $\mathcal{C}$ which is closed under direct summands, that is, $\mathcal{C}^{\mathrm{b}}$ is a thick subcategory of $\mathcal{C}$. For a $\operatorname{ring} \Lambda, \operatorname{Proj}(\Lambda)$ denotes the category of projective $\Lambda$-modules.

LEMMA 5.1. [18]. For any object $T$ in $\mathcal{C}$, the full subcategory $\operatorname{Add}(T)$ is contravariantly finite in $\mathcal{C}$. If $T$ is compact, then the functor $\mathrm{H}_{T}: \mathcal{C} \rightarrow \operatorname{Mod}-\Lambda_{T}^{\mathrm{op}}$ induces an equivalence $\operatorname{Add}(T) \stackrel{\approx}{\rightarrow} \operatorname{Proj}\left(\Lambda_{T}^{\mathrm{op}}\right)$ and the canonical map $\mathcal{C}(X, C) \rightarrow \operatorname{Hom}_{\Lambda_{T}}\left(\mathrm{H}_{T}(X), \mathrm{H}_{T}(C)\right)$ is invertible for any $X \in \operatorname{Add}(T)$ and $C \in \mathcal{C}$.

Following Neeman [66] we say that a triangulated category $\mathcal{C}$ with all small coproducts satisfies Brown's representability theorem if any cohomological functor $F: \mathcal{C}^{\mathrm{op}} \rightarrow \mathcal{A} b$ which converts coproducts to products is representable. The following basic result is essentially due to Krause [52, Theorem 2.2].

THEOREM 5.2. Let $\mathcal{C}$ be a triangulated category satisfying Brown's representability theorem. Then for any compact object $T$ in $\mathcal{C}$ with local endomorphism ring there exists an $A R$-triangle $\Sigma^{-1} E \rightarrow A \rightarrow T \rightarrow E$ in $\mathcal{C}$ and the object $E$ is the $\mathrm{m}$-dual object $\mathbb{D}_{\mathfrak{m}}(T)$ of $T$ where $\mathrm{m}$ is the maximal ideal of $\operatorname{End}_{\mathcal{C}}(T)$.

Proof. Since $T$ is compact, the cohomological functor $\mathrm{H}_{T, \mathrm{~m}}^{\vee}: \mathcal{C}^{\mathrm{op}} \rightarrow \mathcal{A} b$ takes coproducts to products and therefore it is representable. Then the assertion follows from Corollary 3.5.

Compactly generated triangulated categories form an important class of triangulated categories satisfying Brown's representability theorem. Recall that $\mathcal{C}$ is compactly generated, if $\mathcal{C}$ has all small coproducts and admits a generating set which is closed under suspensions and consists of compact objects, see [64]. Compactly generated triangulated categories include the following important examples.

- The unbounded derived category $\mathbf{D}(\operatorname{Mod}-\Lambda)$ of right $\Lambda$-modules over a ring $\Lambda$. The compact objects are the perfect complexes, see e.g. [76, 6.3].

- The stable homotopy category $\mathrm{Ho}(\mathcal{S} p)$ of spectra. The compact objects are the finite spectra, see [61].

- The unbounded derived category $\mathbf{D}(q c \mathbb{X})$ of quasi-coherent sheaves over a quasi-compact separated scheme $\mathbb{X}$. The complexes lying in the thick subcategory of $\mathbf{D}(q c \mathbb{X})$ generated by powers of an ample line bundle form the compact objects, see [64, Proposition 2.5].

- The stable module category Mod- $\Lambda$ modulo projectives of a QF-ring $\Lambda$, e.g. a group algebra $k G$ of a finite group $G$, see [36]. Clearly the compact objects are the finitely generated modules. 
Remark 5.3. More generally well-generated categories in the sense of Neeman [66] or perfectly generated categories in the sense of Krause [54] are examples of triangulated categories satisfying Brown's representability theorem. Note that, by [65, Theorem 0.2], the unbounded derived category $\mathbf{D}(\mathcal{A})$ of a Grothendieck category $\mathcal{A}$ is well-generated. Also if $\mathcal{T}$ is the stable homotopy category of spectra and $E$ is a spectrum, then the full subcategory of $E$-acyclic spectra and the full subcategory of $E$-local spectra are well-generated, see [66, Appendix D]. If $\mathcal{C}$ denotes a triangulated category from the above list, then by Theorem 5.2 it follows that any compact object $T$ in $\mathcal{C}$ is the source of an AR-triangle in $\mathcal{C}$.

Remark 5.4. If $T$ is an object of $\mathcal{C}$ with local endomorphism ring and if the homological functor $\mathrm{H}_{\wedge}^{T, \mathfrak{n}}: \mathcal{C} \rightarrow \mathcal{A} b$ is representable, then, by Remark 3.12, $T$ appears as a source of an AR-triangle in $\mathcal{C}$. If $\mathcal{C}$ is well-generated, then it suffices to know that $\mathrm{H}_{\wedge}^{T, n}$ preserves products, since by [66, Theorem 8.6.1] any product preserving homological functor is representable over a wellgenerated category.

For general, not necessarily compactly or well generated, triangulated categories with all small coproducts we have the following existence result for AR-triangles ending at compact objects. First recall that a thick subcategory $\mathcal{T}$ of $\mathcal{C}$ is called localizing if $\mathcal{T}$ is closed under all small coproducts.

THEOREM 5.5. Let $\mathcal{C}$ be a triangulated category with all small coproducts. If $T$ is an object in $\mathcal{C}$ with local endomorphism ring, then the following are equivalent:

(i) $T$ is compact and there is an AR-triangle $\Sigma^{-1} E \rightarrow A \rightarrow T \rightarrow E$ in $\mathcal{C}$.

(ii) The functor $\mathrm{H}_{T, \mathrm{~m}}^{\vee}:=\operatorname{Hom}_{\Lambda_{T}}\left[\mathrm{H}_{T}(-), I_{\mathrm{m}}\right]: \mathcal{C}^{\mathrm{op}} \rightarrow \mathcal{A}$ b is representable.

Proof. (ii) $\Rightarrow$ (i) By Corollary 3.5 it suffices to show that $T$ is compact. Let $E$ be the representing object of $\mathrm{H}_{T, \mathrm{~m}}^{\vee}$ in $\mathcal{C}$. Let $\left\{C_{i} \mid i \in I\right\}$ be a set of objects in $\mathcal{C}$ and let $\phi: \oplus_{i \in I} \mathrm{H}_{T}\left(C_{i}\right) \rightarrow \mathrm{H}_{T}\left(\oplus_{i \in I} C_{i}\right)$ be the canonical map. Then the composition

$$
\begin{aligned}
& \operatorname{Hom}_{\Lambda_{T}}\left[\mathrm{H}_{T}\left(\bigoplus_{i \in I} C_{i}\right), I_{\mathfrak{m}}\right] \rightarrow \operatorname{Hom}_{\Lambda_{T}}\left[\bigoplus_{i \in I} \mathrm{H}_{T}\left(C_{i}\right), I_{\mathfrak{m}}\right] \\
& \cong \\
& \cong \prod_{i \in I} \operatorname{Hom}_{\Lambda_{T}}\left[\mathrm{H}_{T}\left(C_{i}\right), I_{\mathfrak{m}}\right]
\end{aligned}
$$

is isomorphic to the canonical invertible map $\mathcal{C}\left(\oplus_{i \in I} C_{i}, E\right) \rightarrow \prod_{i \in I} \mathcal{C}\left(C_{i}, E\right)$. Hence the map $\operatorname{Hom}_{\Lambda_{T}}\left(\phi, I_{\mathrm{m}}\right)$ is invertible in Mod- $\Lambda_{T}^{\mathrm{op}}$. Since $I_{\mathrm{m}}$ is an 
injective cogenerator in Mod- $\Lambda_{T}^{\mathrm{op}}$ we conclude that $\phi$ is invertible, so $T$ is compact.

(i) $\Rightarrow$ (ii) By Lemma 3.6, the canonical map $\mathcal{C}(-, E) \stackrel{\omega}{\rightarrow} \operatorname{Hom}_{\Lambda_{T}}\left[\mathrm{H}_{T}(-)\right.$, $\left.\mathrm{H}_{T}(E)\right]$ is injective. Using Lemma 5.1, the proof of Lemma 3.8 shows that $\omega$ is invertible. We show that the left $\Lambda_{T}$-module $\mathrm{H}_{T}(E)$ is injective. Let $\mathcal{T}$ be the localizing subcategory of $\mathcal{C}$ generated by $T$, i.e. $\mathcal{T}$ is the smallest thick subcategory of $\mathcal{C}$ which is closed under all small coproducts and contains $T$. Then $\mathcal{T}$ is compactly generated by $T$ and it is well known that the inclusion $\mathcal{T} \hookrightarrow \mathcal{C}$ admits a right adjoint $\mathrm{R}: \mathcal{C} \rightarrow \mathcal{T}$ which preserves coproducts, see [64, Theorems 4.1 and 5.1]. Since the functor $F_{T}^{\prime}:=\operatorname{Hom}_{\Lambda_{T}}\left[\mathcal{T}(T,-), I_{\mathrm{m}}\right]: \mathcal{T}^{\mathrm{op}} \rightarrow \mathcal{A} b$ is cohomological and sends coproducts to products, by Brown Representability there exists an object $E^{\prime} \in \mathcal{T}$ and a natural isomorphism $\mathcal{T}\left(-, E^{\prime}\right) \stackrel{\cong}{\rightrightarrows} F_{T}^{\prime}$. Then by Corollary 3.5 it follows that there exists an AR-triangle $\Sigma^{-1} E^{\prime} \stackrel{g^{\prime}}{\rightarrow} X \stackrel{f^{\prime}}{\rightarrow} T \stackrel{h^{\prime}}{\rightarrow} E^{\prime}$ in $\mathcal{T}$ and we have an isomorphism $\mathcal{T}\left(T, E^{\prime}\right) \stackrel{\cong}{\rightarrow} I_{\mathfrak{m}}$ by Proposition 2.8. Since $f^{\prime}$ is not split epic there exists a morphism of triangles in $\mathcal{C}$ :

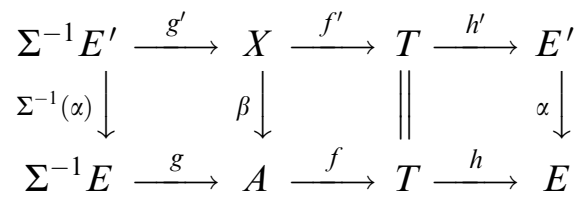

Let $\mathrm{R}(E) \stackrel{\rho}{\rightarrow} E \stackrel{\tau}{\rightarrow} Y \rightarrow \Sigma \mathrm{R}(E)$ be a triangle in $\mathcal{C}$ where $\rho$ is the coreflection of $E$ in $\mathcal{T}$. Applying the functor $\mathcal{C}(T,-)$ to this triangle, we deduce an isomorphism $\mathcal{T}(T, \mathrm{R}(E))=\mathcal{C}(T, \mathrm{R}(E)) \cong \mathcal{C}(T, E)$. Since $E^{\prime}$ lies in $\mathcal{T}$ there exists a unique morphism $\delta: E^{\prime} \rightarrow \mathrm{R}(E)$ such that $\delta \circ \rho=\alpha$. If $\delta$ is not split monic, then since $\mathrm{R}(E)$ lies in $\mathcal{T}$ and $\Sigma\left(g^{\prime}\right)$ is left almost split, it follows that $h^{\prime} \circ \delta=0$. Then $h^{\prime} \circ \delta \circ \rho=\alpha=0$, hence $h=0$ and this is impossible. We infer that $\delta$ is split monic. Hence $\mathrm{R}(E)=E^{\prime} \oplus X^{\prime}$ where $X^{\prime}$ lies in $\mathcal{T}$ since the latter is closed under direct summands. Then we have isomorphisms of left $\Lambda_{T}$-modules: $\mathcal{C}(T, E) \stackrel{\cong}{\rightarrow} \mathcal{T}(T, \mathrm{R}(E))=\mathcal{T}\left(T, E^{\prime}\right) \oplus \mathcal{T}\left(T, X^{\prime}\right)$. By Lemma 3.6, the injective envelope of $\mathcal{C}(T, E)$ is isomorphic to $I_{\mathfrak{m}} \stackrel{\cong}{\rightrightarrows} \mathcal{T}\left(T, E^{\prime}\right)$. This implies that $\mathcal{T}\left(T, X^{\prime}\right)=0$. Hence $\mathrm{H}_{T}(E)=\mathcal{C}(T, E) \stackrel{\cong}{\rightrightarrows} \mathcal{T}\left(T, E^{\prime}\right) \stackrel{\cong}{\rightarrow} I_{\mathfrak{m}}$ and we infer that $I_{\mathfrak{m}}$ is realizable in $\mathcal{C}$ by the object $E$. Then by Proposition 2.8 we have that the functor $\mathrm{H}_{T, \mathrm{~m}}^{\vee}$ is representable.

Remark 5.6. By Theorem 5.5 it follows that for any compact object $T$ in a triangulated category $\mathcal{C}$ which admits infinite sums, there exists a relative m-dual object $\mathbb{D}_{\mathrm{m}}^{\mathcal{T}}(T)$ of $T$ which lies in the localizing subcategory $\mathcal{T}$ of $\mathcal{C}$ generated by $T$ and satisfies $\mathrm{H}_{T, \mathfrak{m}}^{\vee}(-) \stackrel{\cong}{\rightrightarrows} \mathcal{T}\left(\mathrm{R}(-), \mathbb{D}_{\mathfrak{m}}^{\mathcal{T}}(T)\right)$, where $\mathrm{R}$ is the right 
adjoint of the inclusion $\mathcal{T} \hookrightarrow \mathcal{C}$. It follows easily from this that if $T$ appears as a target of an AR-triangle in $\mathcal{C}$, then $\operatorname{R} \tau^{+}(T)=\tau_{\mathcal{T}}^{+}(T)$, where $\tau_{\mathcal{T}}^{+}$denotes Auslander-Reiten translation in $\mathcal{T}$.

Now let $\mathcal{X} \subseteq \mathcal{C}$ be a class of objects. We denote by ${ }^{\perp} \mathcal{X}$ the left, resp. right, orthogonal subcategory of $\mathcal{C}$ defined by $\perp \mathcal{X}:=\{A \in \mathcal{C} \mid \mathcal{C}(A, X)=0$, $\forall X \in \mathcal{X}\}$, resp. $\mathcal{X}^{\perp}:=\{A \in \mathcal{C} \mid \mathcal{C}(X, A)=0, \forall X \in \mathcal{X}\}$

COROLLARY 5.7. Let $T$ be a compact object in $\mathcal{C}$ with local endomorphism ring and let $\mathcal{T}$ be the localizing subcategory of $\mathcal{C}$ generated by $T$. If $(\mathrm{T}): \Sigma^{-1}(E) \stackrel{g}{\rightarrow} A \stackrel{f}{\rightarrow} T \stackrel{h}{\rightarrow} E$ is a triangle in $\mathcal{T}$, then the following are equivalent.

(i) $(\mathrm{T})$ is an AR-triangle in $\mathcal{C}$.

(ii) (T) is an AR-triangle in $\mathcal{T}$ and E lies in $\mathcal{T}^{\perp \perp}$.

In particular if $\tau_{\mathcal{T}}^{+}(T)$ lies in $\mathcal{T}^{\perp \perp}$, for any compact object $T$ in $\mathcal{C}$ with local endomorphism ring, then $\mathcal{C}$ has right AR-triangles ending at compact objects.

Proof. (i) $\Rightarrow$ (ii) Clearly ( $\mathrm{T})$ is an AR-triangle in $\mathcal{T}$. Since $\mathrm{H}_{T, \mathrm{~m}}^{\vee} \cong \mathcal{C}(-, E)$ and $\mathrm{H}_{T, \mathrm{~m}}^{\vee}(Y)=0, \forall Y \in \mathcal{T}^{\perp}$, it follows that $\mathcal{C}(Y, E)=0$, i.e. $E$ lies in $\mathcal{T}^{\perp \perp}$.

(ii) $\Rightarrow$ (i) It suffices to show that any map $\alpha: C \rightarrow T$ in $\mathcal{C}$ which is not a split epimorphism factors through $f$. Let $X_{C} \rightarrow C \rightarrow Y^{C} \rightarrow \Sigma\left(X_{C}\right)$ be a triangle in $\mathcal{C}$ where $X_{C} \in \mathcal{T}$ and $Y^{C} \in \mathcal{T}^{\perp}$. Then the composition $X_{C} \rightarrow C \rightarrow T$ factors through $f$ since it is not a split epimorphism. Hence we have a morphism of triangles:

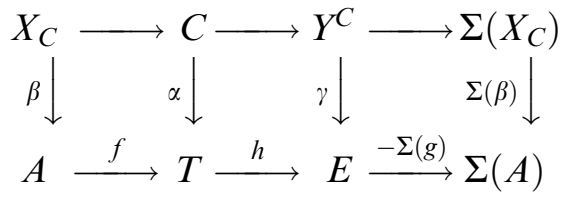

By hypothesis the morphism $\gamma$ is zero and therefore $\alpha$ factors through $f$.

\subsection{AUSLANDER-REITEN TRIANGLES AND TORSION PAIRS}

The above results suggest to study more closely the behavior of AR-triangles with respect to torsion pairs in a triangulated category.

Recall from [22] that a pair $(\mathcal{X}, \mathcal{Y})$ of full strict subcategories of $\mathcal{C}$ is called a torsion pair in $\mathcal{C}$, if: $(\alpha) \mathcal{X}$ and $\mathcal{Y}$ are closed under $\Sigma$ and $\mathcal{C}(X, Y)=0, \forall X \in \mathcal{X}$, $\forall Y \in \mathcal{Y}$, and $(\beta)$ for any object $C$ in $\mathcal{C}$ there exists a triangle $X_{C} \rightarrow C \rightarrow Y^{C} \rightarrow \Sigma\left(X_{C}\right)$ in $\mathcal{C}$ with $X_{C} \in \mathcal{X}$ and $Y^{C} \in \mathcal{Y}$. If $(\mathcal{X}, \mathcal{Y})$ is a torsion pair in $\mathcal{C}$, then $\mathcal{X}$ and $\mathcal{Y}$ are thick subcategories of $\mathcal{C}$, the assignment $C \rightarrow X_{C}$ gives a right adjoint $\mathrm{R}_{\mathcal{X}}: \mathcal{C} \rightarrow \mathcal{X}$ of the inclusion $\mathrm{i}_{\mathcal{X}}: \mathcal{X} \hookrightarrow \mathcal{C}$ and the assignment $C \rightarrow Y^{C}$ gives a left adjoint $\mathrm{L}_{\mathcal{Y}}: \mathcal{C} \rightarrow \mathcal{Y}$ of the inclusion $\mathrm{j}_{\mathcal{Y}}: \mathcal{Y} \hookrightarrow \mathcal{C}$. If $\mathrm{i}_{\mathcal{X}}$, resp. $j_{y}$, also admits a left adjoint, resp. a right adjoint, then the left adjoint of $i_{\mathcal{X}}$ is denoted by $L_{\mathcal{X}}$, resp. the right adjoint of $j_{\mathcal{Y}}$ is denoted by $R_{\mathcal{Y}}$. A triple 
$(\mathcal{X}, \mathcal{Y}, \mathcal{Z})$ of subcategories of $\mathcal{C}$ is called a torsion triple in $\mathcal{C}$, if $(\mathcal{X}, \mathcal{Y})$ and $(\mathcal{Y}, \mathcal{Z})$ are torsion pairs; in this case $\mathcal{X}$ is triangle equivalent to $\mathcal{Z}$, see [22, Corollary I.2.9]. Note that the notion of torsion triple is equivalent to recollement in the sense of [14]. Finally a torsion quadruple in $\mathcal{C}$ is a quadruple $(\mathcal{W}, \mathcal{X}, \mathcal{Y}, \mathcal{Z})$ of subcategories of $\mathcal{C}$ such that $(\mathcal{W}, \mathcal{X}, \mathcal{Y})$ and $(\mathcal{X}, \mathcal{Y}, \mathcal{Z})$ are torsion triples. In this case $\mathcal{W}$, resp. $\mathcal{X}$, is triangle equivalent to $\mathcal{Y}$, resp. $\mathcal{Z}$. For more information on torsion pairs in triangulated categories we refer to [22].

LEMMA 5.8. Let $\mathcal{C}$ be Krull-Schmidt and let $(\mathcal{X}, \mathcal{Y})$ be a torsion pair in $\mathcal{C}$. If $\mathcal{C}$ has right, resp. left, AR-triangles, then $\mathcal{X}$, resp. $\mathcal{Y}$, has right, resp. left, AR-triangles.

Proof. If $\tau_{\mathcal{C}} T \rightarrow A \rightarrow T \rightarrow \Sigma\left(\tau_{\mathcal{C}} T\right)$ is an AR-triangle in $\mathcal{C}$ with $T \in \mathcal{X}$, then clearly the composition $X_{A} \rightarrow A \rightarrow T$ is right almost split in $\mathcal{X}$. Since $\mathcal{X}$ is Krull-Schmidt, by [1, Lemma 2.6], there exists a minimal right almost split map $X \rightarrow T$ in $\mathcal{X}$, hence $\tau_{\mathcal{X}} T \rightarrow X \rightarrow T \rightarrow \Sigma\left(\tau_{\mathcal{X}} T\right)$ is an AR-triangle in $\mathcal{X}$. The case for $\mathcal{Y}$ is similar.

If there exists a torsion triple in $\mathcal{C}$, then we have the following result which gives a nice connection between the end terms of an AR-triangle.

LEMMA 5.9. If $(\mathcal{X}, \mathcal{Y}, \mathcal{Z})$ is a torsion triple in $\mathcal{C}$ and $(\mathrm{T}): E \stackrel{\alpha}{\rightarrow} A \stackrel{\beta}{\rightarrow} T \stackrel{\gamma}{\rightarrow} \Sigma(E)$ is an $A R$-triangle in $\mathcal{C}$, then $T \in \mathcal{X}$ if and only if $E \in \mathcal{Z}$. In particular if $\mathcal{C}$ is Krull$S c h m i d t$ with $A R$-triangles, then all the categories $\mathcal{X}, \mathcal{Y}$ and $\mathcal{Z}$ have AR-triangles. If $\mathcal{C}$ admits a Serre functor $\mathrm{S}$, then $\mathrm{S}$ induces a triangle equivalence $\mathcal{X} \stackrel{\approx}{\rightarrow} \mathcal{Z}$.

Proof. Assume that $T$ lies in $\mathcal{X}$ and consider the morphism $g: E \rightarrow Z^{E}$. If $g$ is not split monic, then $\Sigma^{-1}(\gamma) \circ g$ factors through the fiber $Y_{E} \rightarrow E$ of $g$. Since $\mathcal{C}\left(T, Y_{E}\right)=0$, it follows that $\Sigma^{-1}(\gamma)=0$ and this is impossible. Hence $g$ is a split monic and this implies that $E$ lies in $\mathcal{Z}$. The converse is proved similarly. Assume now that $\mathcal{C}$ is Krull-Schmidt with AR-triangles. Then by Lemma 5.8 it follows that $\mathcal{Y}$ has AR-triangles, $\mathcal{X}$ has right AR-triangles and $\mathcal{Z}$ has left AR-triangles. Since, by [22], $\mathcal{X}$ and $\mathcal{Z}$ are triangle equivalent, both have AR-triangles. If $S$ is a Serre functor in $\mathcal{C}$, then the isomorphisms $\mathrm{DC}(X, Y) \rightarrow \mathcal{C}(Y, \mathrm{~S}(X))$ and $\mathrm{DC}(Y, Z) \rightarrow \mathcal{C}\left(\mathrm{S}^{-1}(Z), Y\right)$ show that $\mathrm{S}:$ $\mathcal{X} \leftrightarrows \mathcal{Z}: \mathrm{S}^{-1}$ are quasi-inverse triangle equivalences.

EXAMPLE 5.10. If $\Lambda$ is a self-injective Artin algebra and $\mathcal{Y}$ is a contravariantly or covariantly finite resolving and coresolving subcategory of mod- $\Lambda$, then by [22, Corollary VI.4.12] there exists a torsion triple $(\mathcal{X}, \mathcal{Y}, \mathcal{Z})$ in the stable category mod- $\Lambda$. So by Lemma 5.9 the subcategories $\mathcal{X}, \mathcal{Y}$ and $\mathcal{Z}$ of mod- $\Lambda$ admit AR-triangles.

In case $\mathcal{C}$ is $R$-finite we have the following more precise result.

PROPOSITION 5.11. Let $\mathcal{C}$ be an R-finite triangulated category over a commutative ring $R$. If $(\mathcal{X}, \mathcal{Y})$ is a torsion pair in $\mathcal{C}$, then we have the following. 
(i) If $\mathcal{C}$ has right $A R$-triangles, then $\mathcal{X}$ has right $A R$-triangles.

(ii) If $\mathcal{C}$ has left AR-triangles, then $\mathcal{Y}$ has left AR-triangles.

(iii) If $\mathcal{C}$ has $A R$-triangles, then the following are equivalent:

(a) $\mathcal{X}$ has left AR-triangles.

(b) $\mathcal{Y}$ has right AR-triangles.

(c) The inclusion $\mathcal{X} \hookrightarrow \mathcal{C}$ admits a left adjoint. Equivalently there exists a torsion triple $(\mathcal{W}, \mathcal{X}, \mathcal{Y})$ in $\mathcal{C}$.

(d) The inclusion $\mathcal{Y} \hookrightarrow \mathcal{C}$ admits a right adjoint. Equivalently there exists a torsion triple $(\mathcal{X}, \mathcal{Y}, \mathcal{Z})$ in $\mathcal{C}$.

If (a) holds, then $\mathcal{X}$ and $\mathcal{Y}$, and all their left or right iterated orthogonal subcategories ${ }^{(n) \perp} \mathcal{X}, \mathcal{X}^{\perp(n)}$ and $\mathcal{Y}^{\perp(n)},{ }^{(n) \perp} \mathcal{Y}$ have AR-triangles.

Proof. Since $\mathcal{C}$ is Krull-Schmidt, parts (i) and (ii) follow from Lemma 5.8.

(iii) Assume that $\mathcal{C}$ has AR-triangles. Then by Theorem 4.9, $\mathcal{C}$ admits a Serre functor $S_{\mathcal{C}}: \mathcal{C} \rightarrow \mathcal{C}$. Let $\mathrm{R}: \mathcal{C} \rightarrow \mathcal{X}$, resp. $L: \mathcal{C} \rightarrow \mathcal{Y}$, be the right, resp. left, adjoint of the inclusion $\mathcal{X} \hookrightarrow \mathcal{C}$, resp. $\mathcal{Y} \hookrightarrow \mathcal{C}$.

(a) $\Rightarrow$ (c) $\Rightarrow$ (b) Since $\mathcal{X}$ has left AR-triangles, by (i), $\mathcal{X}$ has ARtriangles and therefore $\mathcal{X}$ admits a Serre functor $\mathrm{S}_{\mathcal{X}}: \mathcal{X} \rightarrow \mathcal{X}$. Then the isomorphisms

$$
\mathcal{C}(C, X) \stackrel{\cong}{\rightrightarrows} \mathrm{D}^{2} \mathcal{C}(C, X) \stackrel{\cong}{\rightarrow} \mathrm{DC}\left(X, \mathrm{~S}_{\mathcal{C}}(C)\right) \stackrel{\cong}{\rightarrow} \mathrm{D} \mathcal{X}\left(X, \mathrm{RS}_{\mathcal{C}}(C)\right) \stackrel{\cong}{\rightarrow} \mathcal{X}\left(\mathrm{S}_{\mathcal{X}}^{-1} \mathrm{RS}_{\mathcal{C}}(C), X\right)
$$

show that the functor $\mathrm{L}_{\mathcal{X}}:=\mathrm{S}_{\mathcal{X}}^{-1} \mathrm{RS}_{\mathcal{C}}: \mathcal{C} \rightarrow \mathcal{X}$ is a left adjoint of the inclusion $\mathcal{X} \hookrightarrow \mathcal{C}$. Then setting $\mathcal{W}:={ }^{\perp} \mathcal{X}$, we obtain a torsion triple $(\mathcal{W}, \mathcal{X}, \mathcal{Y})$ in $\mathcal{C}$ and by Lemma 5.9 it follows that $\mathcal{Y}$ has right AR-triangles.

(b) $\Rightarrow$ (d) $\Rightarrow$ (a) As in the proof of (a) $\Rightarrow$ (c) it is easy to check that the functor $S_{\mathcal{Y}} \mathrm{LS}_{\mathcal{C}}^{-1}: \mathcal{C} \rightarrow \mathcal{Y}$ is a right adjoint of the inclusion $\mathcal{Y} \hookrightarrow \mathcal{C}$, where $S_{\mathcal{Y}}$ is a Serre functor of $\mathcal{Y}$. Then setting $\mathcal{Z}:=\mathcal{Y}^{\perp}$, we obtain a torsion triple $(\mathcal{X}, \mathcal{Y}, \mathcal{Z})$ in $\mathcal{C}$ and therefore by Lemma 5.9 it follows that $\mathcal{X}$ has left AR-triangles.

By Proposition 5.11 if $\mathcal{C}$ has AR-triangles and $(\mathcal{X}, \mathcal{Y})$ is a torsion pair in $\mathcal{C}$, then $\mathcal{X}$ and $\mathcal{Y}$ have AR-triangles iff there exists a torsion quadruple $(\mathcal{W}, \mathcal{X}, \mathcal{Y}, \mathcal{Z})$ in $\mathcal{C}$. Based on an idea of Bondal and Kapranov [23] we give a converse.

THEOREM 5.12. Let $\mathcal{C}$ be an $R$-finite triangulated category over a commutative ring $R$ and let $\mathcal{X}$ and $\mathcal{Y}$ be thick subcategories of $\mathcal{C}$. Assume that $\mathcal{C}(\mathcal{X}, \mathcal{Y})=0, \mathcal{X}$ and $\mathcal{Y}$ are functorially finite in $\mathcal{C}$ and $\mathcal{C}$ is generated as a triangulated category by $\mathcal{X}$ and $\mathcal{Y}$. Then the following conditions are equivalent.

(i) $\mathcal{C}$ has AR-triangles.

(ii) Both $\mathcal{X}$ and $\mathcal{Y}$ have AR-triangles. 
If (i) holds, then there exist torsion quadruples $\left(\mathcal{O}_{n+2}, \mathcal{O}_{n+1}, \mathcal{O}_{n}, \mathcal{O}_{n-1}\right)$ in $\mathcal{C}$, $\forall n \in \mathbb{Z}$, where $\mathcal{O}_{0}=\mathcal{X}$ and $\mathcal{O}_{-1}=\mathcal{Y}$, and all the categories $\mathcal{O}_{n}$ have $A R$ triangles.

Proof. Since $\mathcal{C}$ is Krull-Schmidt, by [22, Propositions I.2.6 and II.2.4], there exists a torsion quadruple $\left({ }^{\perp} \mathcal{X}, \mathcal{X}, \mathcal{Y}, \mathcal{Y}^{\perp}\right)$ in $\mathcal{C}$. Then part (i) $\Rightarrow$ (ii) follows from Proposition 5.11. Assume now that $\mathcal{X}$ and $\mathcal{Y}$ have AR-triangles and let $\mathrm{s}_{\mathcal{X}}: \mathcal{X} \rightarrow \mathcal{X}$ and $\mathrm{S}_{\mathcal{Y}}: \mathcal{Y} \rightarrow \mathcal{Y}$ be their Serre functors. Also let $\mathrm{R}_{\mathcal{X}}, \mathrm{L}_{\mathcal{X}}: \mathcal{C} \rightarrow \mathcal{X}$ be the right, left adjoint of the inclusion $\mathcal{X} \hookrightarrow \mathcal{C}$ and let $\mathrm{R}_{\mathcal{Y}}, \mathrm{L}_{\mathcal{Y}}: \mathcal{C} \rightarrow \mathcal{Y}$ be the right, left adjoint of the inclusion $\mathcal{Y} \hookrightarrow \mathcal{C}$. Then we have torsion triples $(\mathcal{W}, \mathcal{X}, \mathcal{Y})$ and $(\mathcal{X}, \mathcal{Y}, \mathcal{Z})$ in $\mathcal{C}$, where $\mathcal{W}={ }^{\perp} \mathcal{X}$ and $\mathcal{Z}=\mathcal{Y}^{\perp}$. Let $\mathrm{L}_{\mathcal{Z}}: \mathcal{C} \rightarrow \mathcal{Z}$ be the left adjoint of the inclusion $\mathcal{Z} \hookrightarrow \mathcal{C}$. By Theorem 4.9, to show that $\mathcal{C}$ has AR-triangles, it suffices to construct a Serre functor for $\mathcal{C}$. To this end we follow a construction due to Bondal and Kapranov [23, Theorem 2.10]. Let $C$ be in $\mathcal{C}$. Using adjointness and the existence of Serre functors we have the following isomorphisms:

$$
\begin{aligned}
& \alpha_{C,-}:\left.\mathrm{DC}(C,-)\right|_{\mathcal{X}} \cong \mathrm{D} \mathcal{X}\left(\mathrm{L}_{\mathcal{X}}(C),-\right) \stackrel{\cong}{\rightrightarrows} \mathcal{X}\left(-, \mathrm{S}_{\mathcal{X}} \mathrm{L}_{\mathcal{X}}(C)\right) \\
& \beta_{C,-}:\left.\mathrm{DC}(C,-)\right|_{\mathcal{Y}} \cong \mathrm{D} \mathcal{Y}\left(\mathrm{L}_{\mathcal{Y}}(C),-\right) \stackrel{\cong}{\rightrightarrows} \mathcal{Y}\left(-, \mathrm{S}_{\mathcal{Y}} \mathrm{L}_{\mathcal{Y}}(C)\right)
\end{aligned}
$$

Ignoring the inclusion functors, consider the triangle in $\mathcal{C}$

$$
\mathrm{R}_{\mathcal{Y}} \mathrm{S}_{\mathcal{X}} \mathrm{L}_{\mathcal{X}}(C) \stackrel{g}{\rightarrow} \mathrm{S}_{\mathcal{X}} \mathrm{L}_{\mathcal{X}}(C) \stackrel{f}{\rightarrow} \mathrm{L}_{\mathcal{Z}} \mathrm{S}_{\mathcal{X}} \mathrm{L}_{\mathcal{X}}(C) \stackrel{h}{\rightarrow} \Sigma \mathrm{R}_{\mathcal{Y}} \mathrm{S}_{\mathcal{X}} \mathrm{L}_{\mathcal{X}}(C)
$$

induced from the torsion pair $(\mathcal{Y}, \mathcal{Z})$ and let $\omega$ be the composite morphism:

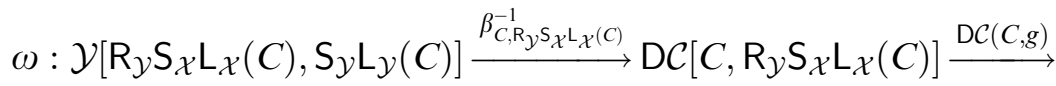

$$
\begin{aligned}
& \mathrm{DC}\left(C, \mathrm{~S}_{\mathcal{Y}} \mathrm{L}_{\mathcal{Y}}(C)\right) \stackrel{\alpha_{C, \mathrm{~S}_{\mathcal{X}} \mathrm{L}_{\mathcal{X}}^{(C)}}}{\longrightarrow} \mathcal{X}\left[\mathrm{S}_{\mathcal{X}} \mathrm{L}_{\mathcal{X}}(C), \mathrm{S}_{\mathcal{X}} \mathrm{L}_{\mathcal{X}}(C)\right] .
\end{aligned}
$$

We set $\rho:=\omega^{-1}\left(1_{\mathrm{S}_{\mathcal{X}} \mathrm{L}_{\mathcal{X}}(C)}\right): \mathrm{R}_{\mathcal{Y}} \mathrm{S}_{\mathcal{X}} \mathrm{L}_{\mathcal{X}}(C) \longrightarrow \mathrm{S}_{\mathcal{Y}} \mathrm{L}_{\mathcal{Y}}(C)$ and consider the triangle

$$
Y(C) \stackrel{\tau}{\rightarrow} \mathrm{R}_{\mathcal{Y}} \mathrm{S}_{\mathcal{X}} \mathrm{L}_{\mathcal{X}}(C) \stackrel{\rho}{\rightarrow} \mathrm{S}_{\mathcal{Y}} \mathrm{L}_{\mathcal{Y}}(C) \stackrel{\sigma}{\rightarrow} \Sigma Y(C)
$$

Then the composition $\tau \circ g$ induces the following octahedron

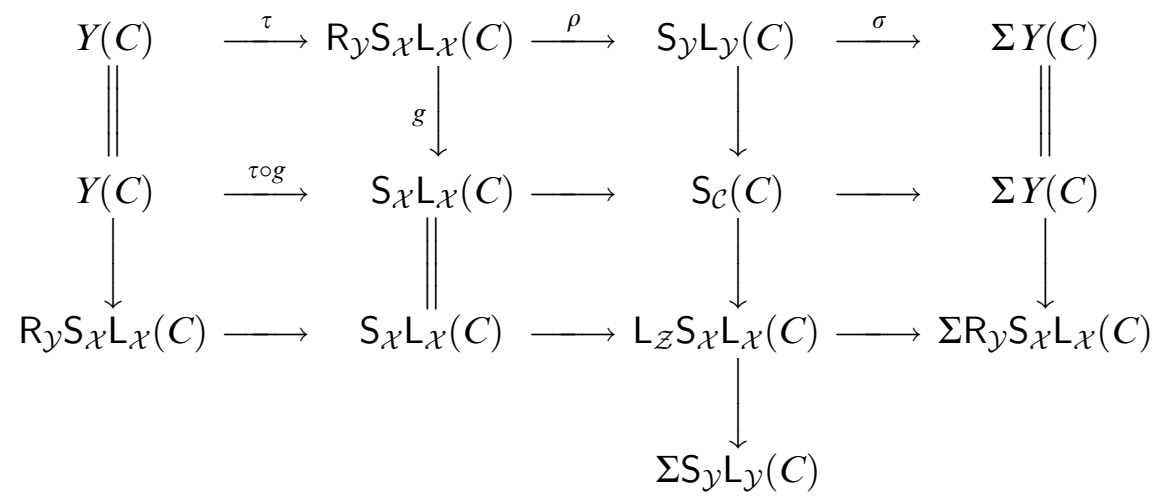


We claim that the object $\mathrm{S}_{\mathcal{C}}(C)$ is the evaluation of a right Serre functor $\mathrm{S}_{\mathcal{C}}: \mathcal{C} \rightarrow \mathcal{C}$ in $\mathcal{C}$ at $C$. Indeed let $A$ be in $\mathcal{C}$ and consider the triangle (again ignoring the inclusion functors) $\left(T_{A}\right): \mathrm{R}_{\mathcal{X}}(A) \rightarrow A \rightarrow \mathrm{L}_{\mathcal{Y}}(A) \rightarrow \Sigma \mathrm{R}_{\mathcal{X}}(A)$ induced by the torsion pair $(\mathcal{X}, \mathcal{Y})$. Applying to this triangle the functor $\mathcal{C}\left(-, \mathrm{S}_{\mathcal{C}}(C)\right)$ we have the exact sequence $\mathcal{C}\left[\mathrm{L}_{\mathcal{Y}}(A), \mathrm{S}_{\mathcal{C}}(C)\right] \rightarrow \mathcal{C}\left[A, \mathrm{~S}_{\mathcal{C}}(C)\right] \rightarrow$ $\mathcal{C}\left[\mathrm{R}_{\mathcal{X}}(A), \mathrm{S}_{\mathcal{C}}(C)\right]$. Using that the object $\mathrm{L}_{\mathcal{Z}} \mathrm{S}_{\mathcal{X}} \mathrm{L}_{\mathcal{X}}(C)$ lies in $\mathcal{Z}=\mathcal{Y}^{\perp}$, we have isomorphisms:

$$
\begin{aligned}
\mathcal{C}\left[\mathrm{L}_{\mathcal{Y}}(A), \mathrm{S}_{\mathcal{C}}(C)\right] & \cong \\
& \cong \mathcal{Y}\left[\mathrm{L} \mathcal{Y}(A), \mathrm{S}_{\mathcal{Y}} \mathrm{L}_{\mathcal{Y}}(C)\right] \stackrel{\cong}{\rightrightarrows} \mathrm{D} \mathcal{Y}\left[C, \mathrm{~L}_{\mathcal{Y}}(A)\right]
\end{aligned}
$$

Similarly since the object $Y(C)$ lies in $\mathcal{Y}=\mathcal{X}^{\perp}$, we have isomorphisms:

$$
\begin{aligned}
& \mathcal{C}\left[\mathrm{R}_{\mathcal{X}}(A), \mathrm{S}_{\mathcal{C}}(C) \stackrel{\cong}{\cong} \mathcal{X}\left[\mathrm{R}_{\mathcal{X}}(A), \mathrm{S}_{\mathcal{X}} \mathrm{L}_{\mathcal{X}}(C)\right] \stackrel{\cong}{\cong} \mathrm{D} \mathcal{X}\left[\mathrm{L}_{\mathcal{X}}(C), \mathrm{R}_{\mathcal{X}}(A)\right]\right. \\
& \stackrel{\cong}{\rightrightarrows} \mathcal{C}\left[C, \mathrm{R}_{\mathcal{X}}(A)\right]
\end{aligned}
$$

Therefore we have an exact sequence $\mathrm{DC}\left[C, \mathrm{~L}_{\mathcal{Y}}(A)\right] \rightarrow \mathcal{C}\left[A, \mathrm{~S}_{\mathcal{C}}(C)\right] \rightarrow$ $\mathrm{DC}\left[C, \mathrm{R}_{\mathcal{X}}(A)\right]$ which is easily seen to be isomorphic to the exact sequence resulting by applying the functor $\mathrm{DC}(C,-)$ to the triangle $\left(T_{A}\right)$. It follows that we have an isomorphism $\mathrm{DC}(C, A) \rightarrow \mathcal{C}\left(A, \mathrm{~S}_{\mathcal{C}}(C)\right)$ which is easily seen to be functorial in both $A$ and $C$, since all the involved constructions are functorial. Therefore $S_{\mathcal{C}}$ is a right Serre functor in $\mathcal{C}$. Working as above with the functor $\mathrm{DC}(-, C)$ we infer in a similar way that $\mathcal{C}$ admits a left Serre functor and consequently $\mathcal{C}$ admits a Serre functor. Finally the last assertion follows by repeated application of Proposition 5.11.

\subsection{TORSION PAIRS OF COMPACT OBJECTS}

In Sections 8 and 9 we are interested in having criteria ensuring that the full subcategory $\mathcal{C}^{\mathrm{b}}$ of compact objects of a compactly generated triangulated category $\mathcal{C}$ has AR-triangles. In this connection torsion pairs provide useful information. We close this section studying the question of when a torsion pair $(\mathcal{X}, \mathcal{Y})$ in $\mathcal{C}$ restricts to a torsion pair $\left(\mathcal{X}^{\mathrm{b}}, \mathcal{Y}^{\mathrm{b}}\right)$ in $\mathcal{C}^{\mathrm{b}}$. This is related to certain finiteness conditions on the torsion pair $(\mathcal{X}, \mathcal{Y})$ which will be useful later in connection with representation embeddings and the Ziegler spectrum.

Recall from [54] that a triangulated category $\mathcal{C}$ is called perfectly generated, resp. cogenerated, if $\mathcal{C}$ has all small coproducts, resp. products, and admits a generating, resp. cogenerating, set $S$ such that for any countable set of maps $A_{i} \rightarrow B_{i}$ in $\mathcal{C}$, the induced map $\mathcal{C}\left(S, \oplus_{i} A_{i}\right) \rightarrow \mathcal{C}\left(S, \oplus_{i} B_{i}\right)$, resp. $\mathcal{C}\left(\prod_{i} B_{i}, S\right) \rightarrow \mathcal{C}\left(\prod_{i} A_{i}, S\right)$, is surjective provided that the maps $\mathcal{C}\left(S, A_{i}\right) \rightarrow$ $\left.\mathcal{C}\left(S, B_{i}\right)\right)$, resp. $\mathcal{C}\left(B_{i}, S\right) \rightarrow \mathcal{C}\left(A_{i}, S\right)$, are surjective for all $i$. In this case $S$ is called a perfect generating, resp. cogenerating, set in $\mathcal{C}$. By recent results of Krause [54, Theorem A] it follows that perfectly generated categories satisfy Brown's Representability Theorem and perfectly cogenerated categories $\mathcal{C}$ 
satisfy Brown's Representability Theorem for the Dual, i.e. product preserving homological functors $\mathcal{C} \rightarrow \mathcal{A} b$ are representable. In the sequel we shall need the following result which is due to Krause, see Proposition 10.1, Corollary 10.2 and their duals in [58].

LEMMA 5.13.

(1) If $\mathcal{C}$ is compactly generated, then $\mathcal{C}$ is perfectly (co)generated.

(2) A triangulated functor $G: \mathcal{C} \rightarrow \mathcal{D}$, where $\mathcal{C}$ is perfectly cogenerated, admits a left adjoint iff $G$ preserves all small products.

(3) A triangulated functor $F: \mathcal{C} \rightarrow \mathcal{D}$, where $\mathcal{C}$ is perfectly generated, admits a right adjoint iff $F$ preserves all small coproducts.

Now let $\mathcal{C}$ be a triangulated category with all small products and coproducts. Recall from [22] that a torsion pair $(\mathcal{X}, \mathcal{Y})$ in $\mathcal{C}$ is of finite type, resp. of cofinite type, if $\mathcal{Y}$ is closed under coproducts, resp. $\mathcal{X}$ is closed under products.

PROPOSITION 5.14. Let $(\mathcal{X}, \mathcal{Y})$ be a torsion pair in a triangulated category $\mathcal{C}$ which admits all small products and coproducts. Then we have the following.

(i) If $\mathcal{C}$ is perfectly cogenerated, then: $(\mathcal{X}, \mathcal{Y})$ is of cofinite type iff there exists a torsion triple $(\mathcal{W}, \mathcal{X}, \mathcal{Y})$ in $\mathcal{C}$. In this case $\mathcal{X}$ is perfectly cogenerated. Moreover if $\mathcal{C}$ is compactly generated, then so is $\mathcal{X}$.

(ii) If $\mathcal{C}$ is perfectly generated, then: $(\mathcal{X}, \mathcal{Y})$ is of finite type iff there exists a torsion triple $(\mathcal{X}, \mathcal{Y}, \mathcal{Z})$ in $\mathcal{C}$. In this case $\mathcal{Y}$ is perfectly generated. Moreover if $\mathcal{C}$ is compactly generated, then so is $\mathcal{Y}$.

(iii) If $\mathcal{C}$ is perfectly generated and cogenerated, then: $(\mathcal{X}, \mathcal{Y})$ is of finite and cofinite type iff there exists a torsion quadruple $(\mathcal{W}, \mathcal{X}, \mathcal{Y}, \mathcal{Z})$ in $\mathcal{C}$. In this case the categories $\mathcal{W}, \mathcal{X}, \mathcal{Y}$, and $\mathcal{Z}$ are perfectly generated and cogenerated. Finally $\mathcal{C}$ is compactly generated iff $\mathcal{W}$ and $\mathcal{X}$ (and $\mathcal{Y}$ and $\mathcal{Z}$ ) are so.

Proof. (i) Clearly the existence of a torsion triple $(\mathcal{W}, \mathcal{X}, \mathcal{Y})$ in $\mathcal{C}$ implies that $\mathcal{X}$ is closed under products. Assume now that $\mathcal{X}$ is closed under products and let $U$ be a perfect cogenerating set in $\mathcal{C}$. We claim that $\mathrm{R}_{\mathcal{X}}(U)$ is a perfect cogenerating set in $\mathcal{X}$, where $\mathrm{R}_{\mathcal{X}}: \mathcal{C} \rightarrow \mathcal{X}$ is the right adjoint of the inclusion $\mathrm{i}_{\mathcal{X}}: \mathcal{X} \hookrightarrow \mathcal{C}$. Indeed if $\mathcal{X}\left(X, \mathrm{R}_{\mathcal{X}}(U)\right)=0$, then by adjointness we have $\mathcal{C}\left(\mathrm{i}_{\mathcal{X}}(X), U\right)=0$ and therefore $X=0$. If $X_{i} \rightarrow X_{i}^{\prime}$ is a countable set of maps in $\mathcal{X}$ such that the induced maps $\mathcal{X}\left(X_{i}^{\prime}, \mathrm{R}_{\mathcal{X}}(U)\right) \rightarrow \mathcal{X}\left(X_{i}, \mathrm{R}_{\mathcal{X}}(U)\right)$ are surjective for all $i$, then by adjointness so are the maps $\mathcal{C}\left(\mathrm{i}_{\mathcal{X}}\left(X_{i}^{\prime}\right), U\right) \rightarrow \mathcal{C}\left(\mathrm{i}_{\mathcal{X}}\left(X_{i}\right), U\right)$. Since $\mathcal{C}$ is perfectly cogenerated, the induced map $\mathcal{C}\left(\prod_{i} \mathrm{i}_{\mathcal{X}}\left(X_{i}^{\prime}\right), U\right) \rightarrow$ $\mathcal{C}\left(\prod_{i} i_{\mathcal{X}}\left(X_{i}\right), U\right)$ is surjective. Since $\mathcal{X}$ is closed under products in $\mathcal{C}$, it follows that $i_{\mathcal{X}}$ preserves products and then using adjointness it follows that the last map is isomorphic to $\mathcal{X}\left(\prod_{i} X_{i}^{\prime}, \mathrm{R}_{\mathcal{X}}(U)\right) \rightarrow \mathcal{X}\left(\prod_{i} X_{i}, \mathrm{R}_{\mathcal{X}}(U)\right)$ hence it is surjective. Hence $\mathrm{R}_{\mathcal{X}}(U)$ is a perfect cogenerating set in $\mathcal{X}$ and then by Lemma 
5.13 it follows that $i_{\mathcal{X}}$ admits a left adjoint. By [22, Proposition I.2.3] this is equivalent to the existence of a torsion pair $(\mathcal{W}, \mathcal{X})$ in $\mathcal{C}$, i.e. we have a torsion triple $(\mathcal{W}, \mathcal{X}, \mathcal{Y})$ in $\mathcal{C}$. Finally if $\mathcal{C}$ is compactly generated and $(\mathcal{W}, \mathcal{X}, \mathcal{Y})$ is a torsion triple in $\mathcal{C}$, then $\mathcal{X}$ is compactly generated by [22, Proposition IV.1.1].

(ii) is similar to (i) and (iii) follows by combining (i), (ii), [22, Corollary IV.1.4] and using that for a torsion triple $(\mathcal{X}, \mathcal{Y}, \mathcal{Z})$ in $\mathcal{C}, \mathcal{X}$ and $\mathcal{Z}$ are triangle equivalent.

We call a torsion triple $(\mathcal{X}, \mathcal{Y}, \mathcal{Z})$ in $\mathcal{C}$ perfect if the torsion pair $(\mathcal{X}, \mathcal{Y})$ is of cofinite type and the torsion pair $(\mathcal{Y}, \mathcal{Z})$ is of finite type. As a consequence of Proposition 5.14 and [22, Proposition IV.1.11] we have the following result which gives sufficient conditions for the existence of torsion pairs or triples in the full subcategory of compact objects.

COROLLARY 5.15. Let $(\mathcal{X}, \mathcal{Y}, \mathcal{Z})$ be a torsion triple in a compactly generated triangulated category $\mathcal{C}$. Then we have the following.

(i) The torsion pair $(\mathcal{Y}, \mathcal{Z})$ is of finite type, i.e. $\mathcal{Z}$ is closed under coproducts in $\mathcal{C}$, iff there exists a torsion quadruple $(\mathcal{X}, \mathcal{Y}, \mathcal{Z}, \mathcal{U})$ in $\mathcal{C}$, in which case the torsion pair $(\mathcal{X}, \mathcal{Y})$ restricts to a torsion pair $\left(\mathcal{X}^{\mathrm{b}}, \mathcal{Y}^{\mathrm{b}}\right)$ in $\mathcal{C}^{\mathrm{b}}$

(ii) The torsion pair $(\mathcal{X}, \mathcal{Y})$ is of cofinite type, i.e. $\mathcal{X}$ is closed under products, iff there exists a torsion quadruple $(\mathcal{W}, \mathcal{X}, \mathcal{Y}, \mathcal{Z})$ in $\mathcal{C}$, in which case the torsion pair $(\mathcal{W}, \mathcal{X})$ in $\mathcal{C}$ restricts to a torsion pair $\left(\mathcal{W}^{\mathrm{b}}, \mathcal{X}^{\mathrm{b}}\right)$ in $\mathcal{C}^{\mathrm{b}}$.

(iii) The torsion triple $(\mathcal{X}, \mathcal{Y}, \mathcal{Z})$ is perfect iff there exists a torsion quintuple $(\mathcal{W}, \mathcal{X}, \mathcal{Y}, \mathcal{Z}, \mathcal{U})$ in $\mathcal{C}$, in which case the torsion triple $(\mathcal{W}, \mathcal{X}, \mathcal{Y})$ restricts to a torsion triple $\left(\mathcal{W}^{\mathrm{b}}, \mathcal{X}^{\mathrm{b}}, \mathcal{Y}^{\mathrm{b}}\right)$ in $\mathcal{C}^{\mathrm{b}}$.

(iv) If the torsion triple $(\mathcal{X}, \mathcal{Y}, \mathcal{Z})$ is perfect, then there exists a torsion quadruple $\left(\mathcal{W}^{\mathrm{b}}, \mathcal{X}^{\mathrm{b}}, \mathcal{Y}^{\mathrm{b}}, \mathcal{Z}^{\mathrm{b}}\right)$ in $\mathcal{C}^{\mathrm{b}}$ iff the torsion pair $(\mathcal{Z}, \mathcal{U})$ is of finite type.

Combining Corollary 5.15 and Theorem 5.12 we deduce the following.

COROLLARY 5.16. Let $\mathcal{C}$ be a compactly generated triangulated $R$-linear category over a commutative ring $R$ and assume that $\mathcal{C}^{\mathrm{b}}$ is $R$-finite. If $(\mathcal{X}, \mathcal{Y}, \mathcal{Z})$ is a perfect torsion triple in $\mathcal{C}$ and $\mathcal{C}^{\mathrm{b}}$ has AR-triangles, then both $\mathcal{X}^{\mathrm{b}}$ and $\mathcal{Y}^{\mathrm{b}}$ have AR-triangles. The converse holds provided that $\mathcal{Z}^{\perp}$ is closed under coproducts in $\mathcal{C}$.

\section{Purity and the Ziegler Spectrum}

In this section we first recall basic facts concerning purity, Brown Representability, and the Ziegler spectrum of a compactly generated triangulated category. For detailed information we refer to $[18,51]$. Next we isolate specific subsets of the Ziegler spectrum which will be useful later in connection with 
Auslander-Reiten theory for compact objects, and we compare Ziegler spectra of module and triangulated categories via various representation embeddings. Finally we study briefly pure Auslander-Reiten triangles in a Brown category.

Throughout this section $\mathcal{C}$ denotes a compactly generated triangulated category. As already mentioned in Section 5, compactly generated triangulated categories satisfy Brown's representability theorem and therefore cohomological functors $\mathcal{C}^{\mathrm{op}} \rightarrow \mathcal{A} b$ taking coproducts to products are representable. Therefore for any compact object $T$ in $\mathcal{C}$ and any maximal left ideal $m$ of the endomorphism ring $\Lambda_{T}$, the functor $\mathrm{H}_{T, m}^{\vee}:=\operatorname{Hom}_{\Lambda_{T}}\left[\mathrm{H}_{T}(-)\right.$, $\left.I_{\mathrm{m}}\right]: \mathcal{C}^{\text {op }} \rightarrow \mathcal{A} b$ is representable, where $I_{\mathrm{m}}$ is the injective envelope of the simple left $\Lambda_{T}$-module $S_{T, \mathfrak{m}}(T):=\Lambda_{T} / \mathrm{m}$ and $\mathrm{H}_{T}:=\mathcal{C}(T,-): \mathcal{C} \rightarrow \operatorname{Mod}-\Lambda_{T}^{\mathrm{op}}$. Hence the m-dual object $\mathbb{D}_{\mathfrak{m}}(T)$ exists in $\mathcal{C}$ for any compact object $T$ in $\mathcal{C}$ and any maximal left ideal $\mathrm{m}$ of $\Lambda_{T}$. In what follows it is useful to consider the restricted Yoneda functor

$$
\mathrm{H}: \mathcal{C} \longrightarrow \text { Mod- } \mathcal{C}^{\mathrm{b}}, \mathrm{H}(A)=\left.\mathcal{C}(-, A)\right|_{\mathcal{C}^{\mathrm{b}}}
$$

Clearly the functor $\mathrm{H}$ is homological, preserves products and coproducts and it is easy to see that its image is contained in the full subcategory of cohomological functors over $\mathcal{C}^{\mathrm{b}}$. Let $S_{T, \mathrm{~m}} \in$ Mod- $\mathcal{C}^{\mathrm{b}}$ be the simple functor $\mathcal{C}^{\mathrm{b}}(-, T) / \mathcal{C}^{\mathrm{b}}(-, T)_{\mathfrak{m}}$ determined by $T$ and $\mathrm{m}$ as in Section 2. Recall that, for a ring $\Lambda, \operatorname{Max}_{l}(\Lambda)$ denotes the space of maximal left ideals of $\Lambda$. The above construction suggests the following definition, referring to [41, Section 3] for a related module theoretic version. We denote by $\operatorname{Iso}\left(\mathcal{C}^{b}\right)$ the set of isoclasses of compact objects and by $\operatorname{Ind}\left(\mathcal{C}^{\mathrm{b}}\right)$ the set of isoclasses of compact objects with local endomorphism ring.

DEFINITION 6.1. The maximal spectrum of $\mathcal{C}$ is the set of all m-dual objects of the isoclass of compact objects:

$$
\operatorname{Max}(\mathcal{C}):=\left\{\mathbb{D}_{\mathfrak{m}}(T) \in \mathcal{C} \mid T \in \operatorname{Iso}\left(\mathcal{C}^{\mathrm{b}}\right) \text { and } \mathrm{m} \in \operatorname{Max}_{l}\left(\Lambda_{T}\right)\right\}
$$

The reduced maximal spectrum $\operatorname{Max}_{\operatorname{lnd}}(\mathcal{C})$ of $\mathcal{C}$ is the subspace of $\operatorname{Max}(\mathcal{C})$ consisting of the m-dual objects of compact objects of $\mathcal{C}$ with local endomorphism ring. We call the objects in $\operatorname{Max}(\mathcal{C})$ maximal points of $\mathcal{C}$.

In the following we collect some consequences of our previous results.

PROPOSITION 6.2. Let $T$ be a compact object in $\mathcal{C}$.

(1) The $\mathrm{m}$-dual object $\mathbb{D}_{\mathfrak{m}}(T)$ has local endomorphism ring.

(2) There exists a triangle $(\mathrm{T}): \Sigma^{-1} \mathbb{D}_{\mathfrak{m}}(T) \rightarrow A \rightarrow T \rightarrow \mathbb{D}_{\mathfrak{m}}(T)$ in $\mathcal{C}$ and the morphism $\Sigma\left(g_{T}\right): \mathbb{D}_{\mathfrak{m}}(T) \rightarrow \Sigma A$ is left almost split in $\mathcal{C}$.

(3) The image of the morphism $\mathrm{H}\left(h_{T}\right): \mathrm{H}(T) \rightarrow \mathrm{H}\left(\mathbb{D}_{\mathfrak{m}}(T)\right)$ in Mod-C ${ }^{\text {b }}$ is the simple functor $S_{T, \mathrm{~m}}$ and the inclusion $S_{T, \mathrm{~m}} \hookrightarrow \mathrm{H}\left(\mathbb{D}_{\mathfrak{m}}(T)\right)$ is an injective envelope. 
(4) $T$ has local endomorphism ring iff $(\mathrm{T})$ is an AR-triangle in $\mathcal{C}$. Hence the $\mathrm{m}$-dual object $\mathbb{D}_{\mathfrak{m}}(T)$ lies in $\operatorname{Max}_{\operatorname{lnd}}(\mathcal{C})$ iff $T$ is the target of an AR-triangle in $\mathcal{C}$.

PROPOSITION 6.3. The set $\operatorname{Max}(\mathcal{C})$ is a cogenerating set in $\mathcal{C}$ and the set $\mathrm{H}(\operatorname{Max}(\mathcal{C}))$ is a cogenerating set of indecomposable injective objects in Mod- $\mathcal{C}^{\mathrm{b}}$. In particular any functor in Mod- $\mathcal{C}^{\mathrm{b}}$ is a subobject of a product of objects from $\mathrm{H}(\operatorname{Max}(\mathcal{C}))$.

\subsection{PURITY}

Recall from $[18,51]$, that a triangle $(\mathrm{T}): A \stackrel{g}{\rightarrow} B \stackrel{f}{\rightarrow} \mathcal{C} \stackrel{h}{\rightarrow} \Sigma(A)$ in $\mathcal{C}$ is called a pure-triangle iff for any compact object $X$, the sequence $0 \rightarrow \mathcal{C}(X, A) \rightarrow$ $\mathcal{C}(X, B) \rightarrow \mathcal{C}(X, C) \rightarrow 0$ is exact in $\mathcal{A} b$. An object $T$ is called pure-projective, resp. pure-injective, if for any pure triangle as above, the induced sequence $0 \rightarrow \mathcal{C}(T, A) \rightarrow \mathcal{C}(T, B) \rightarrow \mathcal{C}(T, C) \rightarrow 0, \quad$ resp. $\quad 0 \rightarrow \mathcal{C}(C, T) \rightarrow \mathcal{C}(B, T) \rightarrow$ $\mathcal{C}(A, T) \rightarrow 0$, is exact in $\mathcal{A} b$. If $(\mathrm{T})$ is a pure-triangle, we call the morphism $g$ a pure-monomorphism, the morphism $f$ a pure-epimorphism and the morphism $h$ phantom. The collection $\mathrm{Ph}(\mathcal{C})$ of all phantom maps in $\mathcal{C}$ is a two sided ideal of $\mathcal{C}$ closed under $\Sigma$ and the class of pure-triangles is a proper class of triangles in $\mathcal{C}$ in the sense of [18]. An object $C$ in $\mathcal{C}$ is pure-projective, resp. pure-injective, iff $\operatorname{Ph}(C,-)=0$, resp. $\operatorname{Ph}(-, C)=0$.

We denote by $\operatorname{PProj}(\mathcal{C})$, resp. $\operatorname{PInj}(\mathcal{C})$, the full subcategory of $\mathcal{C}$ consisting of the pure-projective, resp. pure-injective, objects. We say that $\mathcal{C}$ has enough pure-projective, resp. pure-injective, objects, if any object $C$ in $\mathcal{C}$ is included in a pure triangle $K \rightarrow P \rightarrow C \rightarrow \Sigma(K)$, resp. $C \rightarrow I \rightarrow L \rightarrow \Sigma(C)$, where $P$ is pure-projective, resp. $I$ is pure-injective. A pure-injective envelope of $A \in \mathcal{C}$ is a left minimal pure-monomorphism $g: A \rightarrow E$ with $E$ pureinjective. Then $\mathcal{C}$ has enough pure-projectives and $\operatorname{PProj}(\mathcal{C})=\operatorname{Add}\left(\mathcal{C}^{\mathrm{b}}\right)$ and $\mathcal{C}$ has pure-injective envelopes and $\operatorname{PInj}(\mathcal{C})=\operatorname{Prod}(\operatorname{Max}(\mathcal{C}))$, where the latter is the full subcategory of $\mathcal{C}$ consisting of direct summands of arbitrary products of objects from $\operatorname{Max}(\mathcal{C})$. We refer to [18, Section 11] and [51, Section 1] for details.

There is a nice interplay between the end terms of an AR-triangle. Notice that the module theoretic analog of this interplay was first observed by Herzog [39].

LEMMA 6.4. If $(\mathrm{T})$ is an AR-triangle, then the following are equivalent.

(i) The triangle $(\mathrm{T})$ is not pure.

(ii) The object $C$ is compact.

(iii) The object $A$ is pure-injective.

Proof. (i) $\Rightarrow$ (ii) Since (T) is not pure, it follows that $h$ is not phantom. Hence there exists a compact object $X$ and a morphism $\alpha: X \rightarrow C$ which does 
not factor through $f$. This clearly implies that $\alpha$ is split epic, hence $C$ is compact.

(ii) $\Rightarrow$ (iii) Let $\beta: B \rightarrow A$ be non-zero phantom. Then its cofiber $\gamma$ is not split mono, hence $\gamma$ factors through $g$. This implies that $\Sigma^{-1}(h)$ factors through $\beta$ and therefore it is phantom. Then $\Sigma^{-1}(h)=0$ since $\Sigma^{-1}(C)$ is compact and this is not the case. It follows that $\beta=0$ and consequently there are no non-zero phantom maps into $A$. Hence $A$ is pure-injective.

(iii) $\Rightarrow$ (i) (T) is not pure since it does not splits and $E$ is pure-injective.

Remark 6.5. The previous result as well as large parts of the theory that follows can be generalized to an arbitrary triangulated category equipped with a proper class of triangles $\mathcal{E}$ with enough projectives in the sense of [18].

The restricted Yoneda functor $\mathrm{H}: \mathcal{C} \rightarrow$ Mod- $\mathcal{C}^{\mathrm{b}}$ plays an important role in the study of purity in $\mathcal{C}$ and, as explained in [19], serves as an analog of the functors $\operatorname{Mod}-\left(\bmod -\Lambda^{\mathrm{op}}\right)^{\mathrm{op}} \leftarrow \operatorname{Mod}-\Lambda \rightarrow \operatorname{Mod}-(\bmod -\Lambda) \quad$ defined $\quad$ by $A \otimes_{\Lambda}-\left.\left.\right|_{\text {mod- } \Lambda^{\text {op }}} \leftarrow A \rightarrow \operatorname{Hom}_{\Lambda}(-, A)\right|_{\text {mod- } \Lambda}$ which provide an indispensable tool for the study of purity of modules. More precisely $\mathrm{H}$ identifies the pureprojective objects of $\mathcal{C}$ with the projective functors of Mod- $\mathcal{C}^{\mathrm{b}}$ and the pureinjective objects of $\mathcal{C}$ with the injective functors of Mod- $\mathcal{C}^{\mathrm{b}}$. It follows that the endomorphism ring of an indecomposable pure-injective object of $\mathcal{C}$ is local and, by Proposition 6.3, any object of $\mathcal{C}$ admits a pure monomorphism into a product of objects from the maximal spectrum $\operatorname{Max}(\mathcal{C})$ of $\mathcal{C}$. Further $\mathrm{H}$ reflects isomorphisms and a triangle $A \rightarrow B \rightarrow \mathcal{C} \rightarrow \Sigma A$ in $\mathcal{C}$ is pure iff $0 \rightarrow \mathrm{H}(A) \rightarrow \mathrm{H}(B) \rightarrow \mathrm{H}(C) \rightarrow 0$ is exact in Mod- $\mathcal{C}^{\mathrm{b}}$. Note that since $\mathcal{C}^{\mathrm{b}}$ is triangulated, the cohomological functors $\left\{\mathcal{C}^{\mathrm{b}}\right\}^{\mathrm{op}} \rightarrow \mathcal{A} b$ coincide with the flat functors. Finally any injective functor is flat and the category of flat functors is closed under products in Mod- $\mathcal{C}^{b}$, see $[18,51]$ for more details. In particular we shall need in the sequel the following consequence of [18, Proposition 4.19].

LEMMA 6.6. [18]

(i) For any pure-projective object $P$ in $\mathcal{C}$ the canonical morphism $\mathrm{H}_{P,-}: \mathcal{C}(P,-) \rightarrow \operatorname{Hom}(\mathrm{H}(P), \mathrm{H}(-))$ is invertible. In particular if $P$ is pureprojective and $\mathrm{H}(f): \mathrm{H}(A) \rightarrow \mathrm{H}(P)$ is split epic in Mod- $\mathcal{C}^{\mathrm{b}}$, then so is $f$.

(ii) For any pure-injective object $E$ in $\mathcal{C}$ the canonical morphism $\mathrm{H}_{-, E}: \mathcal{C}(-, E)$ $\rightarrow \operatorname{Hom}(\mathrm{H}(-), \mathrm{H}(E))$ is invertible. In particular if $E$ is pure-injective and $\mathrm{H}(g): \mathrm{H}(E) \rightarrow \mathrm{H}(A)$ is split monic in Mod-C $\mathcal{C}^{\mathrm{b}}$, then so is $g$.

\subsection{THE ZIEGLER SPECTRUM}

By [51], the family of isoclasses of indecomposable pure-injective objects of $\mathcal{C}$ form a set, which is denoted by $\operatorname{Zg}(\mathcal{C})$. Following [53], the set $\mathrm{Zg}(\mathcal{C})$ becomes 
a topological space, called the Ziegler spectrum of $\mathcal{C}$, if we define a topology $\mathcal{T}$, the Ziegler-topology, as follows. Let $\Phi$ be a collection of maps between compact objects. Then $E \in \mathcal{C}$ is called $\Phi$-injective, if for any $\phi: X \rightarrow Y$ in $\Phi$, any morphism $\alpha: X \rightarrow E$ factors through $\phi$. The class of $\Phi$-injective objects of $\mathcal{C}$ is denoted by $\mathbf{U}_{\Phi}$. The closed subsets of $\mathcal{T}$ are defined to be of the form $\mathbf{U}_{\Phi} \cap \mathrm{Zg}(\mathcal{C})$, where $\Phi$ is a collection of morphisms between compact objects. We refer to the work of Garkusha-Prest [32] for a model-theoretic description of the Ziegler topology using pp-formulas of a (multi-sorted) first order language for $\mathcal{C}$. The following remark gives a torsion theoretic description of the Ziegler topology.

Remark 6.7. Let $(\mathcal{X}, \mathcal{Y})$ be a torsion pair of finite type in $\mathcal{C}$. Then, by Proposition 5.14, the torsion pair $(\mathcal{X}, \mathcal{Y})$ is part of a torsion triple $(\mathcal{X}, \mathcal{Y}, \mathcal{Z})$ in $\mathcal{C}$ and $\mathcal{Y}$ is compactly generated. More precisely if $L_{\mathcal{Y}}: \mathcal{C} \rightarrow \mathcal{Y}$ is the left adjoint of the inclusion iy : $\mathcal{Y} \hookrightarrow \mathcal{C}$ and $\mathcal{T}$ is a set of compact generators of $\mathcal{C}$, then $L_{\mathcal{Y}}(\mathcal{T})$ is a set of compact generators for $\mathcal{Y}$. It is not difficult to see that iy induces a closed continuous $\operatorname{map} \operatorname{Zg}(\mathcal{Y}) \hookrightarrow \operatorname{Zg}(\mathcal{C})$, we refer to Theorem 6.13 below for a more general result. By a result of Krause [56] the map $(\mathcal{X}, \mathcal{Y}) \mapsto \operatorname{Zg}(\mathcal{C}) \cap \mathcal{Y}=\operatorname{Zg}(\mathcal{Y})$ gives a bijection between torsion pairs of finite type in $\mathcal{C}$ and closed subsets of $\mathrm{Zg}(\mathcal{C})$.

Clearly the suspension $\Sigma$ induces an homeomorphism $\operatorname{Zg}(\mathcal{C}) \rightarrow \operatorname{Zg}(\mathcal{C})$ and by the above results it follows that we have inclusions $\operatorname{Max}_{\operatorname{lnd}}(\mathcal{C}) \subseteq$ $\operatorname{Max}(\mathcal{C}) \subseteq \operatorname{Zg}(\mathcal{C})$ and an injective $\operatorname{map} \tau^{+}: \operatorname{Ind}\left(\mathcal{C}^{\mathrm{b}}\right) \rightarrow \operatorname{Max}_{\operatorname{lnd}}(\mathcal{C})$ which sends a compact object $T$ with local endomorphism ring to its AR-translate $\tau^{+}(T)=\Sigma^{-1}\left(\mathbb{D}_{\mathfrak{m}}(T)\right)$.

The Ziegler spectrum $\operatorname{Zg}(\Lambda)$ of a ring $\Lambda$ plays a fundamental role in the analysis of the module category Mod- $\Lambda$. Recall that $\mathrm{Zg}(\Lambda)$ is the set of isoclasses of indecomposable pure-injective $\Lambda$-modules equipped with the Ziegler topology, introduced by Ziegler [80] in model-theoretic terms, having as closed sets the subsets $\mathbf{U}_{\Phi} \subseteq \mathrm{Zg}(\Lambda)$, where $\Phi$ is a collection of maps between finitely presented $\Lambda$-modules. We refer to the works of Prest [68,71,72], Herzog [41] and Krause [53] for a comprehensive treatment of the Ziegler spectrum of a module category. Here we discuss briefly the connections between the Ziegler spectrum of $\mathcal{C}$ and the Ziegler spectrum of the endomorphism ring $\Lambda_{T}$ of a compact generator $T$ of $\mathcal{C}$, thus generalizing recent results of Garkusha and Prest [32].

We assume throughout that $T$ satisfies the Toda condition $\mathcal{C}\left(T, \Sigma^{n}(T)\right)=0, \forall n>0$. Then, by the results of [22, Chapter III], $T$ induces a $t$-structure in $\mathcal{C}$ in the sense of [14], with heart $\mathcal{H}(T)=\left\{A \in \mathcal{C} \mid \mathcal{C}\left(T, \Sigma^{n}(A)\right)=\right.$ $0, \forall n \neq 0\}$, and the functor $\mathcal{C}(T,-): \mathcal{H}(T) \rightarrow \operatorname{Mod}-\Lambda_{T}$ is an equivalence. Usually we view this equivalence as an identification $\operatorname{Mod}-\Lambda_{T}=\mathcal{H}(T) \subseteq \mathcal{C}$. Clearly Mod- $\Lambda_{T}$ is closed under products and coproducts in $\mathcal{C}$ and we have 
cohomology functors $\mathrm{H}^{n}: \mathcal{C} \rightarrow$ Mod- $\Lambda_{T}, \forall n \in \mathbb{Z}$, see [14] or [22] for details. If $T$, in addition, satisfies $\mathcal{C}\left(T, \Sigma^{n}(T)\right)=0, \forall n<0$, so that $T$ is a tilting object in $\mathcal{C}$, then $T$ lies in the heart and it is easy to see that we have isomorphisms $\mathrm{H}^{n} \cong \mathcal{C}\left(T, \Sigma^{n}(-)\right): \mathcal{C} \rightarrow \operatorname{Mod}-\Lambda_{T}$, hence the functors $\mathrm{H}^{*}$ preserve products and coproducts. We have the following connection between the Ziegler spectra $\mathrm{Zg}\left(\Lambda_{T}\right)$ and $\mathrm{Zg}(\mathcal{C})$ which generalizes slightly, and is inspired by, a recent result of Garkusha and Prest, see Theorem 7.3 in [32].

PROPOSITION 6.8. Let $T$ be a compact generator of $\mathcal{C}$ with endomorphism ring $\Lambda_{T}$, and assume that $\mathcal{C}\left(T, \Sigma^{n}(T)\right)=0, \forall n>0$. Then we have the following.

(1) For any pure-injective $\Lambda_{T}$-module $M$, the object $\Sigma^{n}(M)$ is pure-injective in $\mathcal{C}, \forall n \in \mathbb{Z}$. If $T$ is a tilting object, then for any pure-injective object $E$ in $\mathcal{C}$, the cohomology object $\mathrm{H}^{n}(E)$ is pure-injective in Mod- $\Lambda_{T}, \forall n \in \mathbb{Z}$.

(2) The sets $\mathcal{U}_{n}:=\left\{\Sigma^{n}(M) \mid M \in \operatorname{Zg}\left(\Lambda_{T}\right\}\right.$ and their disjoint union $\mathcal{U}=\bigsqcup_{n \in \mathbb{Z}} \mathcal{U}_{n}$ are closed subsets of $\mathrm{Zg}(\mathcal{C})$.

(3) If $\mathcal{C}=\mathbf{D}(\mathcal{A})$ is the unbounded derived category of a Grothendieck category $\mathcal{A}$ and $T$ is a tilting object in $\mathbf{D}(\mathcal{A})$ such that $\Lambda_{T}$ is a right coherent ring with finite weak global dimension, then the inclusion $\operatorname{Mod}-\Lambda_{T} \hookrightarrow \mathbf{D}(\mathcal{A})$ induces homeomorphisms $\mathrm{Zg}\left(\Lambda_{T}\right) \rightarrow \mathcal{U}_{n} \subseteq \operatorname{Zg}(\mathbf{D}(\mathcal{A}))$.

Proof. (1) Using that in both Mod- $\Lambda_{T}$ and $\mathcal{C}$ an object $X$ is pure-injective iff for any index set $I$ the summation map $\oplus_{I} X \rightarrow X$ factors through the (pure-)mono $\oplus_{I} X \rightarrow \prod_{I} X$, see [42, Theorem 7.1] and [51, Theorem 1.8], we infer that any pure-injective $\Lambda_{T}$-module is pure-injective in $\mathcal{C}$. If $T$ is a tilting object, then the cohomology functors $\mathrm{H}^{n}$ preserve products and coproducts and therefore send pure-injective objects in $\mathcal{C}$ to pure-injective $\Lambda_{T}$-modules.

(2) Fix $n \in \mathbb{Z}$ and consider the set of morphisms $\Phi_{n}=\left\{\Sigma^{m}(T) \rightarrow\right.$ $0 \mid m \in \mathbb{Z}, m \neq n\}$ in $\mathcal{C}^{\mathrm{b}}$. Then for any object $\Sigma^{n}(M)$ where $M$ is an indecomposable pure-injective $\Lambda_{T}$-module, any morphism $\Sigma^{m}(T) \rightarrow \Sigma^{n}(M)$ is zero and therefore $\mathcal{U}_{n} \subseteq \mathbf{U}_{\Phi_{n}}$. If $E$ lies in $\mathbf{U}_{\Phi_{n}}$, then $\mathcal{C}\left(\Sigma^{m}(T), E\right)=0, \forall m \neq n$. This implies that $\Sigma^{-n}(E)$ lies in the heart and therefore $E \in \mathcal{U}_{n}$. Hence $\mathcal{U}_{n}=\mathbf{U}_{\Phi_{n}}$ and therefore $\mathcal{U}_{n}$ is a closed subset of $\operatorname{Zg}(\mathcal{C})$. Let $\mathcal{O}:=\operatorname{Zg}(\mathcal{C}) \backslash \mathcal{U}$. Clearly $E$ lies in $\mathcal{O}$ iff there exist (at least two) $m, n \in \mathbb{Z}$ with $m \neq n$ such that $\mathcal{C}\left(T, \Sigma^{n}(E)\right) \neq 0$ and $\mathcal{C}\left(T, \Sigma^{m}(E)\right) \neq 0$, that is, iff $E$ lies in $\mathcal{U}_{n}^{c} \cap \mathcal{U}_{m}^{c}$. Hence $\mathcal{O}=\bigcup_{m \neq n}\left(\mathcal{U}_{n}^{c} \cap \mathcal{U}_{m}^{c}\right)$ is open and therefore $\bigsqcup_{n \in \mathbb{Z}} \mathcal{U}_{n}$ is closed in $\mathrm{Zg}(\mathcal{C})$.

(3) By a result of Keller [48], the tilting object $T$ induces a triangle equivalence $\mathbf{D}\left(\operatorname{Mod}-\Lambda_{T}\right) \stackrel{\approx}{\rightarrow} \mathbf{D}(\mathcal{A})$. Since $\Lambda_{T}$ is right coherent of finite weak global dimension, it follows easily that any finitely presented $\Lambda_{T}$-modules becomes a compact object in $\mathbf{D}\left(\operatorname{Mod}-\Lambda_{T}\right)$, hence in $\mathbf{D}(\mathcal{A})$. Using these facts and the description of the Ziegler spectrum of a ring in [53], the assertion is proved as in [32, Theorem 7.3]. 
COROLLARY 6.9. Let $\mathcal{A}$ be a Grothendieck category and assume that $\mathbf{D}(\mathcal{A})$ contains a tilting object $T$. If $\Lambda_{T}=\operatorname{End}(T)$ is right hereditary, then $\operatorname{Zg}(\mathbf{D}(\mathcal{A}))=\bigsqcup_{\mathbb{Z}} \operatorname{Zg}\left(\Lambda_{T}\right)$

Proof. By [63] for any complex $A$ in $\mathbf{D}\left(\operatorname{Mod}-\Lambda_{T}\right)$ we have $A \cong \bigoplus_{n \in \mathbb{Z}} \mathrm{H}^{n}(A)[-n]$. Since $\Lambda_{T}$ is right coherent, the assertion follows from Proposition 6.8 .

COROLLARY 6.10. Let $\mathcal{C}=\mathrm{Ho}(\mathcal{S} p)$ be the stable homotopy category of spectra. Then for any pure-injective spectrum $E$, the stable homotopy group $\pi_{*}(E)$ is a pure-injective abelian group. Moreover $\mathcal{U}_{n}:=\left\{\Sigma^{n}(M) \mid n \in \mathbb{Z}\right\}$ and $\bigsqcup_{n \in \mathbb{Z}} \mathcal{U}_{n}$ are closed subsets of $\mathrm{Zg}(\mathrm{Ho}(\mathcal{S} p))$, where $M$ is one of the following ( $p$ denotes a prime and we identify $M$ with the corresponding Eilenberg-MacLane spectrum):

(i) $\mathbb{Q}$ and one of the Prüfer groups $\mathbb{Z}\left(p^{\infty}\right)$.

(ii) The cyclic groups $\mathbb{Z}\left(p^{n}\right)$.

(iii) The p-adic completion $\widehat{\mathbb{Z}}_{(p)}$ of $\mathbb{Z}$.

Proof. It is well-known that the sphere spectrum $S^{0}$ is a compact generator of $\mathrm{Ho}(\mathcal{S} p)$ satisfying the condition $\left[S^{0}, \Sigma^{n}\left(S^{0}\right)\right]=0, \forall n>0$, and the heart of the $t$-structure induced by $S^{0}$ coincides with $\mathcal{A} b$, see [22, Section III.3], [61, Theorem 6.1]. Then the assertion follows by Proposition 6.8 and the wellknown classification of indecomposable pure-injective abelian groups, see the book of Kaplansky [45].

\subsection{REPRESENTATION EMBEDDINGS AND THE ZIEGLER SPECTRUM}

Let as before $\mathcal{C}$ be a compactly generated triangulated category. We have seen in Remark 6.7 that if $(\mathcal{X}, \mathcal{Y})$ is a torsion pair of finite type in $\mathcal{C}$ then $\mathcal{Y}$ is compactly generated and the inclusion functor $\mathcal{Y} \rightarrow \mathcal{C}$ preserves products and coproducts. Such functors are special cases of representation embeddings (defined below) which provide a natural tool for comparing Ziegler spectra. Notice that a module theoretic variant of the notion of representation embedding was first introduced by Prest, see [69].

DEFINITION 6.11. A triangulated functor $G: \mathcal{C} \rightarrow \mathcal{D}$ between compactly generated triangulated categories is called definable if $G$ preserves products and coproducts. A definable functor is called a representation embedding if $G$ reflects isomorphisms and preserves indecomposability.

We denote by Flat $\left(\mathcal{C}^{\text {b }}\right)$ the full subcategory of Mod- $\mathcal{C}^{\text {b }}$ consisting of the flat (= cohomological) functors $\left\{\mathcal{C}^{\mathrm{b}}\right\}^{\mathrm{op}} \rightarrow \mathcal{A} b$. The category Flat $\left(\mathcal{C}^{\mathrm{b}}\right)$ is 
locally finitely presented with products in the sense of Crawley-Boevey [27] and exactly definable in the sense of Krause [49]. A theory of purity for such categories were developed in [27,49,71]. In particular one can define the Ziegler spectrum of $\operatorname{Flat}\left(\mathcal{C}^{\mathrm{b}}\right)$ as the set of indecomposable pure-injective objects of Flat $\left(\mathcal{C}^{\mathrm{b}}\right)$ equipped with the Ziegler topology which is defined in a similar way as the Ziegler topology of $\mathcal{C}$, using that the compact objects of $\mathcal{C}$ are the finitely presented objects in $\operatorname{Flat}\left(\mathcal{C}^{b}\right)$. We refer to [71] and [49] for more details. In the sequel we shall need the following result.

LEMMA 6.12. ([51], Proposition 2.6). If $G: \mathcal{C} \rightarrow \mathcal{D}$ is a definable functor, then $G$ preserves pure-injective objects and admits a left adjoint $F$ which preserves compact objects. Moreover there exist an adjoint pair $\left(F_{\mathrm{b}}^{*}, F_{*}^{\mathrm{b}}\right)$ : Mod- $\mathcal{D}^{\mathrm{b}} \leftrightarrows$ Mod- $\mathcal{C}^{\mathrm{b}}$ of colimit preserving exact functors which preserve flat functors and make the following diagrams commutative $\left(\mathrm{H}_{\mathcal{C}}\right.$ and $\mathrm{H}_{\mathcal{D}}$ are the restricted Yoneda functors):

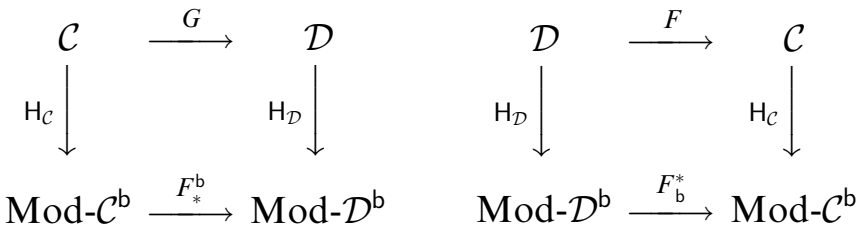

In particular there exists an adjoint pair $\left(F_{\mathrm{b}}^{*}, F_{*}^{\mathrm{b}}\right): \operatorname{Flat}\left(\mathcal{D}^{\mathrm{b}}\right) \leftrightarrows \operatorname{Flat}\left(\mathcal{C}^{\mathrm{b}}\right)$, where $F_{*}^{\mathrm{b}}$ preserves filtered colimits and products and $F_{\mathrm{b}}^{*}$ preserves finitely presented objects.

Proof. By results of Neeman [64, Theorem 5.1] and [66, Theorem 8.6.1] it follows that $G$ admits a left adjoint $F$ which preserves compact objects. Then the existence of the above diagrams and the preservation of pure-injectives by $F$ is proved in [51, Proposition 2.6]. The last assertion follows as in [18, Section 11.4].

After these preliminaries we can prove the following result which generalizes a result of Prest, see [69, Theorem 7], and gives a connection between the Ziegler spectra of categories related by a representation embedding.

THEOREM 6.13. A representation embedding $G: \mathcal{C} \rightarrow \mathcal{D}$ induces a homeomorphic embedding of $\mathrm{Zg}(\mathcal{C})$ as a closed subset of $\mathrm{Zg}(\mathcal{D})$.

Proof. By Lemma 6.12 it follows that $G$ induces an injective function $\mathbf{g}: \operatorname{Zg}(\mathcal{C}) \rightarrow \operatorname{Zg}(\mathcal{D}), \mathbf{g}(E)=G(E)$, and admits a left adjoint $F: \mathcal{D} \rightarrow \mathcal{C}$ which restricts to a functor $F^{\mathrm{b}}: \mathcal{D}^{\mathrm{b}} \rightarrow \mathcal{C}^{\mathrm{b}}$. Let $\delta: \operatorname{Id}_{\mathcal{D}} \rightarrow G F$ be the unit and $\varepsilon: F G \rightarrow \mathrm{Id}_{\mathcal{C}}$ the counit of the adjoint pair $(F, G)$. Let $\Psi$ be a collection of maps in $\mathcal{D}^{\mathrm{b}}$, hence $F(\Psi)=\{F(\psi) \mid \phi \in \Psi\}$ is a collection of maps in $\mathcal{C}^{\mathrm{b}}$, and let $E$ be in $\mathbf{g}^{-1}\left(\mathbf{U}_{\Psi}\right)$, i.e. $G(E)$ is in $\mathbf{U}_{\Psi}$. Also let $F(\psi): F(X) \rightarrow F(Y)$ be a map in 
$F(\Psi)$. Since $G(E)$ is $\Psi$-injective, for any map $\alpha: F(X) \rightarrow E$, there exists a map $\rho: Y \rightarrow G(E)$ such that $\delta_{X} \circ G(\alpha)=\psi \circ \rho$. Applying $F$ and using adjointness, we have: $\quad \alpha=F\left(\delta_{X}\right) \circ \varepsilon_{F(X)} \circ \alpha=F\left(\delta_{X}\right) \circ F G(\alpha) \circ \varepsilon_{E}=F(\psi) \circ F(\rho) \circ \varepsilon_{E}$. Hence $\alpha$ factors through $F(\psi)$ and this shows that $\mathbf{g}^{-1}\left(\mathbf{U}_{\Psi}\right) \subseteq \mathbf{U}_{F(\Psi)}$. Next let $E$ be in $\mathbf{U}_{F(\Psi)}$ and let $\psi: X \rightarrow Y$ be a map in $\Psi$. Since $E$ is $F(\Psi)$-injective, for any map $\beta: X \rightarrow G(E)$, the composition $F(\alpha) \circ \varepsilon_{E}: F(X) \rightarrow E$ factors through $F(\psi)$, say as $F(\phi) \circ \sigma=F(\alpha) \circ \varepsilon_{E}$. Applying $G$ and using adjointness, we have: $\beta=\beta \circ \delta_{G(E)} \circ G\left(\varepsilon_{E}\right)=\delta_{X} \circ G F(\beta) \circ G\left(\varepsilon_{E}\right)=\delta_{X} \circ G F(\psi) \circ G\left(\varepsilon_{E}\right)=$ $\psi \circ \delta_{Y} \circ G(\sigma)$ and this shows that $\beta$ factors through $\psi$. Hence $G(E)$ is $\Psi$-injective and therefore $E$ lies in $\mathbf{g}^{-1}\left(\mathbf{U}_{\Psi}\right)$. Hence $\mathbf{U}_{F(\Psi)} \subseteq \mathbf{g}^{-1}\left(\mathbf{U}_{\Psi}\right)$. Since the closed subsets of $\mathrm{Zg}(\mathcal{C})$, resp. $\operatorname{Zg}(\mathcal{D})$, are of the form $\mathbf{U}_{\Phi}$, resp. $\mathbf{U}_{\Psi}$, where $\Phi$, resp. $\Psi$, are collection of maps in $\mathcal{C}^{\mathrm{b}}$, resp. $\mathcal{D}^{\mathrm{b}}$, we infer that $\mathbf{g}$ is continuous. To show that $\mathbf{g}$ is closed we consider the adjoint pair $\left(F_{\mathrm{b}}^{*}, F_{*}^{\mathrm{b}}\right): \operatorname{Flat}\left(\mathcal{D}^{\mathrm{b}}\right) \leftrightarrows \operatorname{Flat}\left(\mathcal{C}^{\mathrm{b}}\right)$ from Lemma 6.12. Since the functor $\mathrm{H}_{\mathcal{C}}$, resp. $\mathrm{H}_{\mathcal{D}}$, induces an equivalence between the full subcategories of pure-injective objects of $\mathcal{C}$, resp. $\mathcal{D}$, and $\operatorname{Flat}\left(\mathcal{C}^{\mathrm{b}}\right)$, resp. $\operatorname{Flat}\left(\mathcal{D}^{\mathrm{b}}\right)$, it is easy to see that it induces a map between the Ziegler spectra of $\mathcal{C}$, resp. $\mathcal{D}$, and Flat $\left(\mathcal{C}^{\mathrm{b}}\right)$, resp. Flat $\left(\mathcal{D}^{\mathrm{b}}\right)$, which sends Ziegler closed subsets of $\mathcal{C}$, resp. $\mathcal{D}$, to Ziegler closed subsets of $\operatorname{Flat}\left(\mathcal{C}^{\mathrm{b}}\right)$, resp. Flat $\left(\mathcal{D}^{\mathrm{b}}\right)$, see [56, Section 7]. Let $\mathbf{U}_{\Phi}$ be a closed subset of $\mathrm{Zg}(\mathcal{C})$. Then $\mathrm{H}_{\mathcal{C}}\left(\mathbf{U}_{\Phi}\right)$ is a closed subset of $\mathrm{Zg}\left(\operatorname{Flat}\left(\mathcal{C}^{\mathrm{b}}\right)\right)$. By [49, Theorem 7.8] the subset $F_{*}^{\mathrm{b}}\left(\mathrm{H}_{\mathcal{C}}\left(\mathbf{U}_{\Phi}\right)\right)=\mathrm{H}_{\mathcal{D}}\left(G\left(\mathbf{U}_{\Phi}\right)\right)$ is closed in $\mathrm{Zg}\left(\operatorname{Flat}\left(\mathcal{D}^{\mathrm{b}}\right)\right)$ and therefore $G\left(\mathbf{U}_{\Phi}\right)$ is closed in $\operatorname{Zg}(\mathcal{D})$. We infer that the map $\mathrm{g}$ is closed.

We close this subsection by pointing out some consequences.

COROLLARY 6.14. Let $(\mathcal{X}, \mathcal{Y})$ be a torsion pair of finite, resp. cofinite, type in $\mathcal{C}$. Then the inclusion $\mathrm{i}_{\mathcal{Y}}: \mathcal{Y} \hookrightarrow \mathcal{C}$, resp. $\mathrm{i}_{\mathcal{X}}: \mathcal{X} \hookrightarrow \mathcal{C}$, induces a closed homeomorphic embedding $\mathrm{Zg}(\mathcal{Y}) \rightarrow \operatorname{Zg}(\mathcal{C})$, resp. $\mathrm{Zg}(\mathcal{X}) \rightarrow \operatorname{Zg}(\mathcal{C})$.

Proof. By Corollary 5.15, $\mathcal{Y}$, resp. $\mathcal{X}$, is compactly generated and the inclusion $i_{\mathcal{Y}}$, resp. $i_{\mathcal{X}}$, is a representation embedding. So the claim follows by Theorem 6.13.

EXAMPLE 6.15. Let $T$ be a finitely presented module with finite projective dimension over an Artin algebra $\Lambda$ such that $\operatorname{Ext}_{\Lambda}^{n}(T, T)=0, \forall n \geqslant 1$. If the projective dimension of $T$ over $\Gamma:=\operatorname{End}_{\Lambda}(T)$ is finite, then by [22, Propositions IV.1.11 and IV.3.5] there exists a perfect torsion pair $(\mathcal{X}, \mathcal{Y})$ in $\mathbf{D}(\operatorname{Mod}-\Lambda)$, where $\mathcal{X}$ is triangle equivalent to $\mathbf{D}(\operatorname{Mod}-\Gamma)$, so Corollary 6.14 applies.

Recall from [33] that a ring homomorphism $\Lambda \rightarrow \Gamma$ is called a homological epimorphism if the canonical map $\Gamma \otimes_{\Lambda} \Gamma \rightarrow \Gamma$ is invertible and $\operatorname{Tor}_{n}^{\Lambda}(\Gamma, \Gamma)=0, \forall n \geq 1$. The following result gives a derived version to a result of Prest [70, Corollary 9]. 
COROLLARY 6.16. If $\Lambda \rightarrow \Gamma$ is a homological epimorphism of rings, then there exists a homeomorphic embedding of $\mathrm{Zg}(\mathbf{D}(\operatorname{Mod}-\Gamma))$ as a closed subset of $\operatorname{Zg}(\mathbf{D}(\operatorname{Mod}-\Lambda))$.

Proof. By [58, Theorem 14.5] the functor $F:=-\otimes_{\Lambda}^{\mathbb{L}} \Gamma: \mathbf{D}(\operatorname{Mod}-\Lambda) \rightarrow$ $\mathbf{D}($ Mod- $\Gamma)$ admits a coproduct preserving fully faithful right adjoint $G$. Clearly $G$ is a representation embedding and therefore the assertion follows from Theorem 6.13.

COROLLARY 6.17. Let $p$ be a prime and let $\mathrm{Ho}(\mathcal{S} p)$, resp. $\mathrm{Ho}(\mathcal{S} p)_{p}$, be the stable homotopy category of, resp. p-local, spectra. Then there exists a homeomorphic embedding of $\mathrm{Zg}\left(\mathrm{Ho}(\mathcal{S} p)_{p}\right)$ as a closed subset of $\mathrm{Zg}(\mathrm{Ho}(\mathcal{S} p))$.

Proof. By [61, Chapter 8] it follows that the inclusion $\mathrm{Ho}(\mathcal{S} p)_{p} \subseteq \mathrm{Ho}(\mathcal{S} p)$ is a representation embedding and the assertion follows from Theorem 6.13 .

\subsection{PURE AND GHOST AUSLANDER-REITEN TRIANGLES IN BROWN CATEGORIES}

In this subsection we are interested in pure AR-triangles $E \rightarrow A \rightarrow X \rightarrow \Sigma E$ in $\mathcal{C}$. Then, by Lemma $6.4, X \rightarrow \Sigma E$ is phantom, $X$ is not compact and $E$ is not pure-injective. Recall that $\mathcal{C}$ is called a Brown category if the functor $\mathrm{H}: \mathcal{C} \rightarrow$ Mod- $\mathcal{C}^{\mathrm{b}}$, whose strict image lies in the subcategory $\operatorname{Flat}\left(\mathcal{C}^{\mathrm{b}}\right)$ of flat functors, is full [18]. Note that, by [18, Theorem 11.18], for a Brown category $\mathcal{C}$, the composition of two phantom maps is zero and the functor $\mathrm{H}: \mathcal{C} \rightarrow \operatorname{Flat}\left(\mathcal{C}^{\mathrm{b}}\right)$ is surjective on objects. Clearly $\operatorname{Flat}\left(\mathcal{C}^{\mathrm{b}}\right)$ is an exact subcategory of Mod- $\mathcal{C}^{b}$ in the sense of Quillen [73], so that we can speak of Auslander-Reiten sequences in $\operatorname{Flat}\left(\mathcal{C}^{b}\right)$.

THEOREM 6.18. Let $\mathcal{C}$ be a Brown category. If $(\mathrm{T}): E \stackrel{g}{\rightarrow} A \stackrel{f}{\rightarrow} X \stackrel{h}{\rightarrow} \Sigma E$ is a triangle in $\mathcal{C}$, then the following are equivalent.

(i) $E \rightarrow A \rightarrow X \rightarrow \Sigma E$ is a pure AR-triangle in $\mathcal{C}$.

(ii) $0 \rightarrow \mathrm{H}(E) \rightarrow \mathrm{H}(A) \rightarrow \mathrm{H}(X) \rightarrow 0$ is an AR-sequence in $\operatorname{Flat}\left(C^{b}\right)$.

Proof. (i) $\Rightarrow$ (ii) Since the triangle ( $\mathrm{T})$ is pure and the functor $\mathrm{H}$ is homological, full and reflects isomorphisms, the sequence $\mathrm{H}(\mathrm{T}): 0 \rightarrow \mathrm{H}(E) \rightarrow$ $\mathrm{H}(A) \rightarrow \mathrm{H}(X) \rightarrow 0$ is exact and not split in Flat $\left(\mathcal{C}^{\mathrm{b}}\right)$. Let $\widetilde{\alpha}: F \rightarrow \mathrm{H}(X)$ be nonsplit epic in $\operatorname{Flat}\left(\mathcal{C}^{\mathrm{b}}\right)$. Since $\mathrm{H}$ is full and surjective on objects, it follows that $F \cong \mathrm{H}(B)$ and $\mathrm{H}(\alpha)=\widetilde{\alpha}$, where $\alpha: B \rightarrow X$ is a morphism in $\mathcal{C}$. Since $\mathrm{H}(\alpha)$ is nonsplit epic, the same is true for $\alpha$. Hence $\alpha$ factors through $f$, and then $\mathrm{H}(\alpha)$ factors through $\mathrm{H}(f)$. We infer that $\mathrm{H}(f)$ is right almost split. Since $\mathcal{C}$ is Brown, the endomorphism rings $\operatorname{End}(\mathrm{H}(E))$ and $\operatorname{End}(\mathrm{H}(X))$ are local as factor rings of the 
local endomorphism rings $\operatorname{End}(E)$ and $\operatorname{End}(X)$. We conclude that $\mathrm{H}(\mathrm{T})$ is an AR-sequence in Flat $\left(\mathcal{C}^{\mathrm{b}}\right)$.

(ii) $\Rightarrow$ (i) Consider the AR-sequence $0 \rightarrow \mathrm{H}(E) \rightarrow \mathrm{H}(A) \rightarrow \mathrm{H}(X) \rightarrow 0$ in Flat $\left(\mathcal{C}^{\mathrm{b}}\right)$. Since $g$ is pure-mono, the triangle $(\mathrm{T})$ is pure. For any object $A$ in $\mathcal{C}$, the kernel of the ring epimorphism $\operatorname{End}(A) \rightarrow \operatorname{End}(\mathrm{H}(A))$ is the ideal $\mathrm{Ph}(A)$ of self-phantom maps of $A$. By [18], we have $\mathrm{Ph}^{2}(A)=0$, in particular $\mathrm{Ph}(A) \subseteq \operatorname{Jac}(\operatorname{End}(A))$. This implies that if $\operatorname{End}(\mathrm{H}(A)$ is local, then the same is true for $\operatorname{End}(A)$. Consequently the endomorphism rings $\operatorname{End}(E)$ and $\operatorname{End}(X)$ are local. Let $\alpha: B \rightarrow X$ be a morphism in $\mathcal{C}$ which is non-split epic. If $\mathrm{H}(\alpha)$ is split epic, then there exists a morphism $\widetilde{\beta}=\mathrm{H}(\beta): \mathrm{H}(X) \rightarrow \mathrm{H}(B)$ such that $\mathrm{H}(\beta) \circ \mathrm{H}(\alpha)=1_{\mathrm{H}(X)}$. Since $\mathrm{H}$ reflects isomorphisms, we infer that $\alpha$ is split epic and this is not the case. It follows that $\mathrm{H}(\alpha)$ is non-split epic and therefore there exists a morphism $\tilde{\gamma}=\mathrm{H}(\gamma): \mathrm{H}(B) \rightarrow \mathrm{H}(A)$ such that $\mathrm{H}(\gamma) \circ \mathrm{H}(f)=\mathrm{H}(\alpha)$. This implies that the morphism $\gamma \circ f-\alpha: B \rightarrow X$ is phantom. Since $h$ is phantom and the ideal of phantom maps is square zero, we infer that $(\gamma \circ f-\alpha) \circ h=\alpha \circ h=0$. Hence $\alpha$ factors through $f$ and therefore $(\mathrm{T})$ is a pure AR-triangle in $\mathcal{C}$.

COROLLARY 6.19. If $\mathcal{C}$ is Brown, then $\mathrm{H}: \mathcal{C} \rightarrow$ Mod- $\mathcal{C}^{\mathrm{b}}$ induces a bijection

$$
\mathrm{H}: \operatorname{pART}(\mathcal{C}) \leftrightarrow \operatorname{ARS}\left(\operatorname{Flat}\left(\mathcal{C}^{\mathrm{b}}\right)\right)
$$

between the family $\mathrm{pART}(\mathcal{C})$ of isoclasses of pure AR-triangles in $\mathcal{C}$ and the family $\operatorname{ARS}\left(\operatorname{Flat}\left(\mathcal{C}^{b}\right)\right)$ of isoclasses of $A R$-sequences in the exact category Flat $\left(\mathcal{C}^{b}\right)$. In particular if $\operatorname{Flat}\left(\mathcal{C}^{b}\right)$ admits an $A R$-sequence, then $\mathcal{C}$ admits a pure AR-triangle.

The above results admit relative versions. Let $S$ be a compact object in $\mathcal{C}$ and let $\mathcal{E}(S)$ be the proper class of triangles in $\mathcal{C}$ generated by $S$ in the sense of [18]. That is a triangle $A \rightarrow B \rightarrow \mathcal{C} \rightarrow \Sigma(A)$ lies in $\mathcal{E}(S)$ if $0 \rightarrow \mathcal{C}\left(\Sigma^{*}(S), A\right) \rightarrow$ $\mathcal{C}\left(\Sigma^{*}(S), B\right) \rightarrow \mathcal{C}\left(\Sigma^{*}(S), C\right) \rightarrow 0$ is exact, where $\left.\Sigma^{*}(S):=\left\{\Sigma^{n}(S) \mid n \in \mathbb{Z}\right\}\right)$. We call $\mathcal{E}(S)$ the class of ghost triangles in $\mathcal{C}$ with respect to $S$ and recall from $[18$, Lemma 8.1] that the ghost projective objects of $\mathcal{C}$ are the objects of the full subcategory $\operatorname{Add}\left(\Sigma^{*}(S)\right)$. Then as in Propositions 6.2 it follows that any non-ghost-projective compact object $X$ in $\mathcal{C}$ with local endomorphism ring occurs as a target of a ghost AR-triangle in $\mathcal{C}$, and any non-ghostinjective pure-injective object $E$ in $\mathcal{C}$ with local endomorphism ring such that $E$ is reduced maximal point in the Ziegler spectrum occurs as a source of a ghost triangle in $\mathcal{C}$. Let $\mathrm{H}_{S}: \mathcal{C} \rightarrow \operatorname{Mod}-\Sigma^{*}(S), \mathrm{H}_{S}(A)=\left.\mathcal{C}(-, A)\right|_{\Sigma^{*}(S)}$ be the induced homological functor. We say that $\mathcal{C}$ is ghost Brown with respect to $S$ if $\mathrm{H}_{S}$ is full, see [18, Theorem 10.2] for other equivalent conditions. The following is a ghost analogue of Theorem 6.18 and is proved in the same way, using [18]. 
THEOREM 6.20. Assume that $\mathcal{C}$ is a ghost Brown category with respect to the compact object $S$. Then $\operatorname{ImH}_{S}$ is closed under extensions in $\operatorname{Mod}-\Sigma^{*}(S)$. If $E \rightarrow A \rightarrow X \rightarrow \Sigma E$ is a triangle in $\mathcal{C}$, then the following conditions are equivalent.

(i) $E \rightarrow A \rightarrow X \rightarrow \Sigma E$ is a ghost AR-triangle in $\mathcal{C}$.

(ii) $0 \rightarrow \mathrm{H}_{S}(E) \rightarrow \mathrm{H}_{S}(A) \rightarrow \mathrm{H}_{S}(X) \rightarrow 0$ is an AR-sequence in the exact subcategory $\operatorname{ImH}_{S}$ of $\operatorname{Mod}-\Sigma^{*}(S)$.

Since for a right hereditary ring $\Lambda$, the derived category $\mathbf{D}(\operatorname{Mod}-\Lambda)$ is clearly ghost Brown with respect to $\Lambda$ (see Section 12.5 in [18]), we have the following.

COROLLARY 6.21. Let $\Lambda$ be a right hereditary ring. If $E \rightarrow A \rightarrow X \rightarrow \Sigma E$ is a triangle in $\mathbf{D}(\operatorname{Mod}-\Lambda)$, then the following conditions are equivalent.

(i) $E \rightarrow A \rightarrow X \rightarrow \Sigma E$ is a ghost AR-triangle in $\mathbf{D}(\operatorname{Mod}-\Lambda)$.

(ii) $0 \rightarrow \mathrm{H}_{\Lambda}(E) \rightarrow \mathrm{H}_{\Lambda}(A) \rightarrow \mathrm{H}_{\Lambda}(X) \rightarrow 0$ is an AR-sequence in the exact subcategory $\mathrm{ImH}_{\Lambda}$ of $\prod_{\mathbb{Z}}$ Mod- $\Lambda$.

(iii) There exists a unique $n \in \mathbb{Z}$ such that $0 \rightarrow \mathrm{H}^{n}(E) \rightarrow \mathrm{H}^{n}(A) \rightarrow \mathrm{H}^{n}(X) \rightarrow 0$ is an AR-sequence in Mod- $\Lambda$.

Pure homological algebra in a compactly generated triangulated category $\mathcal{C}$ is the analogue of the classical relative homological theory of purity in a module category. In turn ghost homological algebra in $\mathcal{C}$ is an analogue of the absolute homological theory in a module category, see [18, Subsections 12.4 and 12.5] and [21] for more details. Concerning Auslander-Reiten theory these analogies raises some questions. We do not know if in $\mathcal{C}$ there exists a pure or pure-ghost AR-triangle $\Sigma^{-1}(E) \rightarrow A \rightarrow T \rightarrow E$, equivalently an AR-triangle such that $T$ is not compact or $E$ is not pure-injective. For Brown categories Corollary 6.19 shows that the question is equivalent to the existence of an AR-sequence in the category of flat functors. Also Corollary 6.19 raises the question, which we leave open, of the existence of a pure AR-sequence in a module category.

Finally let $\mathrm{Ho}(\mathcal{S} p)_{p}$ be the stable homotopy category of $p$-local spectra where $p$ is a prime [61]. It is easy to see that if Freyd's Generating Hypothesis [28] fails in $\operatorname{Ho}(\mathcal{S} p)_{p}$, then there exists a ghost $\mathrm{AR}$-triangle in $\mathrm{Ho}(\mathcal{S} p)_{p}$ with respect to the $p$-local sphere spectrum $S_{p}^{0}$, ending at a finite $p$-local spectrum.

\section{Maximal Points and Almost Split Morphisms}

Throughout $\mathcal{C}$ denotes a compactly generated triangulated category. In this section we are interested in finding sufficient conditions ensuring that a pureinjective object in $\mathcal{C}$ with local endomorphism ring, is the source or target of a (minimal) left or right almost split morphism or an Auslander-Reiten triangle 
in $\mathcal{C}$, and to characterize the internal structure of these objects in terms of the Ziegler spectrum. Our results here generalize analogous results from ring theory, see [26] and [53].

We begin with the following result which generalizes module theoretic results of Crawley-Boevey [26] and Krause [53] and characterizes the pureinjective objects which occur as a source of a left almost split morphism in $\mathcal{C}$. The proof of the first four conditions is contained in [19, Theorem 7.9]. For completeness we include a simpler proof. To prove the rest we follow ideas of Krause [53].

THEOREM 7.1. If $E$ is an object in $\mathcal{C}$, then the following are equivalent.

(i) $E$ is pure-injective and a source of a left almost split morphism in $\mathcal{C}$.

(ii) $E$ is a source of a left almost split morphism in $\mathcal{C}$ which is not pure-mono.

(iii) $\mathrm{H}(E)$ is the injective envelope in Mod- $\mathcal{C}^{\mathrm{b}}$ of a simple functor.

(iv) $E \in \operatorname{Max}(\mathcal{C})$, that is, there exists a compact object $X$, a maximal left ideal $\mathrm{m}$ of $\operatorname{End}_{\mathcal{C}}(X)$, and an isomorphism $E \cong \mathbb{D}_{\mathfrak{m}}(X)$.

(v) $E$ is pure-injective and if $E$ is a direct summand of a product $\prod_{i \in I} E_{i}$ of indecomposable objects in $\mathcal{C}$, then there exists $i \in I$ such that: $E \cong E_{i}$.

(vi) $E$ is pure-injective and if $E$ is a direct summand of a product $\prod_{i \in I} E_{i}$ of points in $\mathrm{Zg}(\mathcal{C})$, then there exists $i \in I$ such that: $E \cong E_{i}$.

Proof. (i) $\Leftrightarrow$ (ii) Part (i) $\Rightarrow$ (ii) is trivial. If $g: E \rightarrow A$ is a left almost split map which is not pure-mono and $E$ is not pure-injective, then the pureinjective envelope $\mu: E \rightarrow I$ of $E$ is not split mono, hence there exists $\rho: A \rightarrow I$ such that $g \circ \rho=\mu$. Clearly $g$ is pure-mono and this is not the case. So $E$ is pure-injective.

(ii) $\Rightarrow$ (iii) Let $g: E \rightarrow A$ be a left almost split morphism which is not pure-mono, and let $B \stackrel{h}{\rightarrow} E \stackrel{g}{\rightarrow} A \stackrel{f}{\rightarrow} \Sigma B$ be a triangle in $\mathcal{C}$. Let $\varepsilon \circ \mu: \mathrm{H}(B) \rightarrow S \longmapsto \mathrm{H}(E)$ be the canonical factorization of $\mathrm{H}(h)$ where $S=\operatorname{ImH}(h)$. Since $g$ is not pure-mono, it follows that $\mathrm{H}(h) \neq 0$, hence $S \neq 0$. We claim that $S$ is a simple functor. To show this let $\alpha: S \rightarrow F$ be a non-zero map in Mod- $\mathcal{C}^{\mathrm{b}}$, and let $v: F \longmapsto \mathrm{H}(I)$ be an injective envelope. Since $\mathrm{H}(I)$ is injective, $\varepsilon \circ \alpha \circ v$ is of the form $\mathrm{H}(\rho)$ where $\rho: B \rightarrow I$. Then $\mathrm{H}\left(\Sigma^{-1} f\right) \circ \mathrm{H}(\rho)=0$, hence $\Sigma^{-1} f \circ \rho=0$ as a phantom map into the pureinjective object $I$. Therefore there exists a map $\sigma: E \rightarrow I$ such that $h \circ \sigma=\rho$, and it follows directly that $\mu \circ \mathrm{H}(\sigma)=\alpha \circ v$. If $\sigma$ is split mono, then $\alpha$ is a monomorphism. Otherwise, since $g$ is left almost split, there exists a map $\tau: A \rightarrow I$ such that $g \circ \tau=\sigma$. This implies clearly that $\alpha=0$ and this is not the case. We infer that any non-zero map $S \rightarrow F$ is a monomorphism, i.e. $S$ is simple. Since $E$ is pure-injective, the inclusion $S \hookrightarrow \mathrm{H}(E)$ is an injective envelope. 
(iii) $\Rightarrow$ (iv) Since any simple functor over $\mathcal{C}^{\mathrm{b}}$ is of the form $S_{X, \mathrm{~m}}$ where $X$ is compact and $\mathrm{m}$ is a maximal left ideal of $\operatorname{End}_{\mathcal{C}}(X)$ and since, by Proposition 6.2, the injective envelope of $S_{X, \mathfrak{m}}$ is $\mathrm{H}\left(\mathbb{D}_{\mathfrak{m}}(X)\right)$, it follows that $\mathrm{H}\left(\mathbb{D}_{\mathfrak{m}}(X)\right)$ is isomorphic to $\mathrm{H}(E)$. By Lemma 6.6 this implies that $E \cong \mathbb{D}_{\mathfrak{m}}(X)$, that is, $E$ lies in $\operatorname{Max}(\mathcal{C})$.

(iv) $\Rightarrow$ (v) $\Rightarrow$ (vi) If the m-dual object $\mathbb{D}_{\mathfrak{m}}(X)$ is a direct summand of $\prod_{i \in I} E_{i}$, then the injective envelope $\mathrm{H}\left(\mathbb{D}_{\mathfrak{m}}(X)\right)$ of the simple functor $S_{X, \mathfrak{m}}$ is a direct summand of $\mathrm{H}\left(\prod_{i \in I} E_{i}\right)=\prod_{i \in I} \mathrm{H}\left(E_{i}\right)$. Hence $\left(S_{X, \mathfrak{m}}, \mathrm{H}\left(E_{i}\right)\right) \neq 0$ for some $i \in I$. Consequently there exists a non-zero morphism $S_{X, \mathfrak{m}} \rightarrow \mathrm{H}\left(\mathbb{D}_{\mathfrak{m}}(X)\right) \rightarrow \prod_{i \in I} \mathrm{H}\left(E_{i}\right) \rightarrow \mathrm{H}\left(E_{i}\right)$ which is necessarily a monomorphism. Since the inclusion $S_{X, \mathfrak{m}} \rightarrow \mathrm{H}\left(\mathbb{D}_{\mathfrak{m}}(X)\right)$ is essential it follows that $\mathrm{H}\left(\mathbb{D}_{\mathfrak{m}}(X)\right) \rightarrow \mathrm{H}\left(E_{i}\right)$ is a split monomorphism. Using Lemma 6.6 and the indecomposability of $E_{i}$ we infer that $\mathbb{D}_{\mathfrak{m}}(X) \cong E_{i}$.

(vi) $\Rightarrow$ (i) By Proposition 6.3, $E$ is a direct summand of a product of copies of m-dual objects of compacts. Then by hypothesis $E$ is isomorphic to some $\mathbb{D}_{\mathfrak{m}}(X)$. Hence by Proposition $6.2, E$ is the source of a left almost split morphism in $\mathcal{C}$.

The following consequence of Theorem 7.1 characterizes the points in $\operatorname{Max}_{\operatorname{lnd}}(\mathcal{C})$.

COROLLARY 7.2. For an object $E$ in $\mathcal{C}$ the following conditions are equivalent.

(i) $E$ is pure-injective and there exists an AR-triangle $E \rightarrow A \rightarrow X \rightarrow \Sigma(E)$.

(ii) $E \in \operatorname{Max}_{\operatorname{lnd}}(\mathcal{C})$, that is: $E \cong \Sigma^{-1} \mathbb{D}_{\mathfrak{m}}(X)$ where $X$ is compact with local endomorphism ring and $\mathrm{m}$ is a maximal left ideal of $\operatorname{End}_{\mathcal{C}}(X)$.

(iii) $E$ is pure-injective and a source of a left almost split morphism in $\mathcal{C}$, and there exists a compact object $X$ with $\operatorname{End}_{\mathcal{C}}(X)$ local, such that $\mathcal{C}(X, E) \neq 0$.

(iv) $\mathrm{H}(E)$ is the injective envelope of a simple functor $S$, and $S$ admits a projective cover.

Proof. (i) $\Leftrightarrow$ (ii) If $E$ is pure-injective, then Lemma 6.4 implies that $X$ is compact. Therefore $E \cong \mathbb{D}_{\mathfrak{m}}\left(\Sigma^{-1}(X)\right)$ since the end terms of an AR-triangle are uniquely determined up to isomorphism. The converse follows from Proposition 6.2.

(ii) $\Rightarrow$ (iii) The proof is trivial since by construction we have $\mathcal{C}\left(X, \mathbb{D}_{\mathfrak{m}}(X)\right) \neq 0$.

(iii) $\Rightarrow$ (iv) By Theorem 7.1 the functor $\mathrm{H}(E)$ is the injective envelope of a simple functor $S_{T, \mathfrak{m}}$. Since $\mathcal{C}(X, E) \neq 0$, we infer that $S_{T, \mathfrak{m}}(X) \neq 0$. Then clearly the induced non-zero morphism $\mathrm{H}(X) \rightarrow S_{T, \mathrm{~m}}$ is a projective cover.

(iv) $\Rightarrow$ (i) Consider the composition $\mathrm{H}(T) \stackrel{\varepsilon}{\rightarrow} S \stackrel{\mu}{\rightarrow} \mathrm{H}(E)$ where $\varepsilon$ is a projective cover and $\mu$ is an injective envelope of the simple functor $S$. Then 
clearly $T$ has local endomorphism ring and if $h: T \rightarrow E$ is the unique morphism with $\mathrm{H}(h)=\varepsilon \circ \mu$, then the triangle $\Sigma^{-1}(E) \rightarrow A \rightarrow T \rightarrow E$ is an AR-triangle in $\mathcal{C}$.

COROLLARY 7.3. Let $g: E \rightarrow A$ be a left almost split morphism in $\mathcal{C}$. If $A$ is pure-injective, then $E$ is the source of an AR-triangle in $\mathcal{C}$.

Proof. Follows as in Theorem 3.4 using that there exists a decomposition $(g, 0): E \rightarrow A^{\prime} \oplus A^{\prime \prime}=A$ of $g$ such that $g^{\prime}$ is left minimal, see [60, Corollary $1.3]$.

COROLLARY 7.4. If $\mathcal{C}^{\mathrm{b}}$ is Krull-Schmidt, then the following are equivalent.

(i) $E$ is a pure-injective source of a left almost split morphism in $\mathcal{C}$.

(ii) $E$ is a pure-injective source of a minimal left almost split morphism in $\mathcal{C}$.

(iii) $E$ is a pure-injective source of an AR-triangle in $\mathcal{C}$.

(iv) $E$ is a reduced maximal point, that is, $E \cong \mathbb{D}_{\mathfrak{m}}(X)$ where $X$ is an indecomposable compact object and $\mathrm{m}$ is a maximal left ideal of $\operatorname{End}_{\mathcal{C}}(X)$.

Recall that we have an inclusion of spaces $\operatorname{Max}_{\operatorname{lnd}}(\mathcal{C}) \subseteq \operatorname{Max}(\mathcal{C})$. There is an important class of categories for which we have an equality $\operatorname{Max}(\mathcal{C})=\operatorname{Max}_{\text {Ind }}(\mathcal{C})$.

LEMMA 7.5. If $\mathcal{C}^{\mathrm{b}}$ is Krull-Schmidt, then $\operatorname{Max}(\mathcal{C})=\operatorname{Max}_{\operatorname{lnd}}(\mathcal{C})$ ). In particular a point $E \in \operatorname{Zg}(\mathcal{C})$ is maximal iff $E$ is the source of an AR-triangle in $\mathcal{C}$.

Proof. Let $\mathbb{D}_{\mathfrak{m}}(X) \in \operatorname{Max}(\mathcal{C})$, where $X$ is compact and $m$ is a maximal left ideal of $\operatorname{End}_{\mathcal{C}}(X)$. Then $\mathbb{D}_{\mathfrak{m}}(X)$ is the injective envelope of the simple functor $S_{X, \mathrm{~m}}$. Since $\mathcal{C}^{\mathrm{b}}$ is Krull-Schmidt, there exists a (unique) indecomposable direct summand $Y$ of $X$ such that $S_{X, m}(Y) \neq 0$. By Yoneda's Lemma, any non-zero element of $S_{X, \mathfrak{m}}(Y)$ induces an epimorphism $\varepsilon: \mathrm{H}(Y) \rightarrow S_{X, \mathfrak{m}}$ which is a projective cover since $\operatorname{End}(\mathrm{H}(Y))$ is local. Then $\operatorname{Ker}(\varepsilon)$ is the unique maximal submodule $\mathrm{H}(Y)_{\mathfrak{n}}$ of $\mathrm{H}(Y)$, where $\mathfrak{n}$ is the unique maximal ideal of $\operatorname{End}_{\mathcal{C}}(Y)$. Hence $S_{X, \mathrm{~m}} \cong S_{Y, \mathrm{n}}$. Taking injective envelopes it follows that $\mathbb{D}_{\mathfrak{m}}(X) \cong \mathbb{D}_{\mathfrak{n}}(Y) \in \operatorname{Max}_{\text {Ind }}(\mathcal{C})$.

The module theoretic version of the following result was observed by Herzog, see the discussion before Proposition 3.6 in [41].

PROPOSITION 7.6. $\operatorname{Max}(\mathcal{C})$ is a dense subset of $\operatorname{Zg}(\mathcal{C})$.

Proof. It suffices to show that for any closed set $\mathbf{U}_{\Phi}$ in $\mathrm{Zg}(\mathcal{C})$, where $\Phi$ is a family of morphisms in $\mathcal{C}^{\text {b }}$, the inclusion $\operatorname{Max}(\mathcal{C}) \subseteq \mathbf{U}_{\Phi}$ implies that $\mathbf{U}_{\Phi}=\operatorname{Zg}(\mathcal{C})$. Let $\phi: Z \rightarrow Y$ be an arbitrary morphism in $\Phi$ and let $F$ be the kernel of $\mathrm{H}(\phi)$. Since any element of $\operatorname{Max}(\mathcal{C})$ is $\phi$-injective, it follows directly that $\left[F, \mathrm{H}\left(\mathbb{D}_{\mathfrak{m}}(X)\right)\right]=0$, for any compact object $X$ and any maximal left 
ideal $m$ of $\operatorname{End}_{\mathcal{C}}(X)$. By Proposition 2.1 we have an isomorphism $\left[F, \mathrm{H}\left(\mathbb{D}_{\mathfrak{m}}(X)\right)\right] \cong \operatorname{Hom}_{\Lambda_{X}}\left(F(X), I_{\mathfrak{m}}\right)$ of left $\Lambda_{X}$-modules. Fixing the compact object $X$, it follows that $\operatorname{Hom}_{\Lambda_{X}}\left(F(X), I_{\mathfrak{m}}\right)=0$, for any maximal left ideal $\mathrm{m}$ of $\Lambda_{X}$ and therefore $F(X)=0$. Since this happens for any compact object $X$, it follows that $F=0$. Then obviously $\phi$ is a split monomorphism, since $\mathrm{H}(Z)$ is injective in mod- $\mathcal{C}^{\mathrm{b}}$. It follows that $\Phi$ consists of split monomorphisms in $\mathcal{C}^{\mathrm{b}}$ and consequently $\mathbf{U}_{\Phi}=\operatorname{Zg}(\mathcal{C})$. Hence $\operatorname{Max}(\mathcal{C})$ is a dense subset.

We recall that a point $E$ of $\operatorname{Zg}(\mathcal{C})$ is called isolated, if $\{E\}$ is open in $\operatorname{Zg}(\mathcal{C})$. If $\mathcal{O} \subseteq \mathrm{Zg}(\mathcal{C})$, then $\overline{\mathcal{O}}$ denotes the closure of $\mathcal{O}$ in the Ziegler topology. We denote by $\operatorname{Isol}(\mathcal{C})$ the set of isolated points of the Ziegler spectrum $\mathrm{Zg}(\mathcal{C})$. Since $\operatorname{Max}(\mathcal{C})$ is dense in $\operatorname{Zg}(\mathcal{C})$, we have the following consequence.

COROLLARY 7.7. Isol $(\mathcal{C}) \subseteq \operatorname{Max}(\mathcal{C})$, that is: if $E$ is an isolated point of $\operatorname{Zg}(\mathcal{C})$, then $E \cong \mathbb{D}_{\mathfrak{m}}(X)$, for some compact object $X$ of $\mathcal{C}$ and a maximal left ideal $\mathrm{m}$ of $\operatorname{End}_{\mathcal{C}}(X)$. In particular $E$ is the source of a left almost split morphism in $\mathcal{C}$.

COROLLARY 7.8. Let $E=\mathbb{D}_{\mathfrak{m}}(X)$ be a point in the maximal spectrum $\operatorname{Max}(\mathcal{C})$. If the simple functor $S_{X, m}$ is finitely presented, then $E$ is an isolated point of $\mathrm{Zg}(\mathcal{C})$. In particular if all simple functors are finitely presented, then $\operatorname{Max}(\mathcal{C})=\operatorname{Isol}(\mathcal{C})$

Proof. Let $\mu: S_{X, \mathrm{~m}} \longmapsto \mathrm{H}(E)$ be an injective envelope, and let $\mathrm{H}(Z) \rightarrow \mathrm{H}(X) \rightarrow S_{X, \mathfrak{m}} \rightarrow 0$ be a finite presentation of $S_{X, \mathfrak{m}}$, where $f: Z \rightarrow X$ is a map in $\mathcal{C}^{\mathrm{b}}$. Then we have a triangle $Y \stackrel{g}{\rightarrow} Z \stackrel{f}{\rightarrow} X \stackrel{h}{\rightarrow} \Sigma Y$ in $\mathcal{C}^{\mathrm{b}}$ and a factorization $\mathrm{H}(h)=\varepsilon \circ \kappa: \mathrm{H}(X) \stackrel{\varepsilon}{\rightarrow} S_{X, \mathrm{~m}} \stackrel{\kappa}{\rightarrow} \mathrm{H}(\Sigma(Y))$. We set $\Phi:=\{\Sigma g\}$. Let $M \in \operatorname{Zg}(\mathcal{C}) \backslash\{E\}$ and let $\alpha: \Sigma Y \rightarrow M$ be any map. If the composition $\kappa \circ \mathrm{H}(\alpha): S_{X, \mathfrak{m}} \rightarrow \mathrm{H}(M)$ is monic, then there exists a map $\mathrm{H}(\rho): \mathrm{H}(E) \rightarrow$ $\mathrm{H}(M)$ such that $\mu \circ \mathrm{H}(\rho)=\kappa \circ \mathrm{H}(\alpha)$. Since $\mu$ is essential, $\mathrm{H}(\rho)$ split monic and this implies that $\mathrm{H}(E) \cong \mathrm{H}(M)$ since $\mathrm{H}(E)$ and $\mathrm{H}(M)$ are indecomposable. Then $E \cong M$ and this is impossible. We infer that $\kappa \circ \mathrm{H}(\alpha)=0$, and this clearly implies that $\mathrm{H}(h) \circ \mathrm{H}(\alpha)=0$, hence $h \circ \alpha=0$. Therefore $\alpha$ factors through $\Sigma(g)$. This shows that any point in $\operatorname{Zg}(\mathcal{C}) \backslash\{E\}$ is $\Phi$-injective, hence $\mathrm{Zg}(\mathcal{C}) \backslash\{E\}$ is closed in $\operatorname{Zg}(\mathcal{C})$. We infer that $E$ is isolated.

\section{Auslander-Reiten Triangles with Compact End Terms and Finite Points}

Let $\mathcal{C}$ be a compactly generated triangulated category. Our aim in this section is to investigate when the full subcategory $\mathcal{C}^{\mathrm{b}}$ of compact objects of $\mathcal{C}$ has (left or right) AR-triangles in connection with the structure of the Ziegler spectrum. The connection between Auslander-Reiten theory and the Ziegler spectrum in the setting of module categories was first observed by Mike Prest who used a combination of representation and model theoretic methods, see [68]. Later this fruitful connection was investigated further by Herzog [39, 41], Krause [53] and others. 


\subsection{PURE-INJECTIVE ENVELOPES OF COMPACT OBJECTS}

In the study of AR-theory in $\mathcal{C}^{\text {b }}$, an important role is played by the structure of pure-injective envelopes of compact object with local endomorphism ring. We begin their study with the following two preliminary results, observed independently with a different proof by Garkusha-Prest [32, Theorem 2.3], which will be useful later. The module theoretic analogues are due to Prest, see $[68, \S 11.3]$ and the Example in Herzog [41, p. 535].

LEMMA 8.1. For a compact object $W$, the following are equivalent:

(i) $W$ has local endomorphism ring.

(ii) The pure-injective envelope $E(W)$ of $W$ is indecomposable.

Proof. (ii) $\Rightarrow$ (i) If $W \stackrel{\mu}{\rightarrow} E(W) \stackrel{\kappa}{\rightarrow} B \stackrel{\xi}{\rightarrow} \Sigma(W)$ is a triangle where $\mu$ is a pureinjective envelope, then any map $0 \neq \alpha: W \rightarrow W$ induces a morphism of triangles:

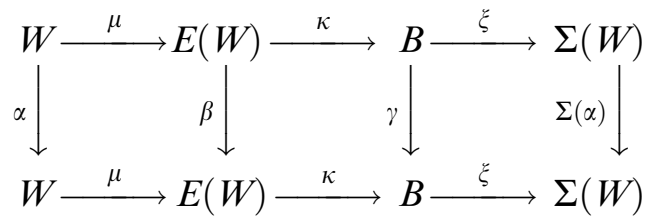

Then $\operatorname{End}(E(W))$ is local since indecomposable pure-injective objects have local endomorphism ring. Hence $\beta$ or $1_{E(W)}-\beta$ is invertible. Assume first that $\beta$ is invertible and let $\beta^{\prime}$ be its inverse. Then $\mu=\mu \circ \beta \circ \beta^{\prime}=\alpha \circ \mu \circ \beta^{\prime}$. Since $\mu$ is a pure-monomorphism, so is $\alpha$ and therefore any triangle $W \stackrel{\alpha}{\rightarrow} W \rightarrow Z \rightarrow \Sigma(W)$ is pure. Since $W$ is compact, so is $Z$ and therefore $\alpha \circ \alpha^{\prime}=1_{W}$ for some morphism $\alpha^{\prime}: W \rightarrow W$. Then the idempotent morphism $e:=\alpha^{\prime} \circ \alpha$ is embedded in a triangle

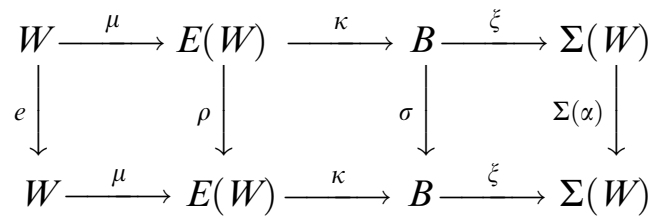

If $\rho$ is in invertible with inverse $\rho^{\prime}$, then $\mu=e \circ \mu \circ \rho^{\prime}$ and as above we infer that $e$ is a pure-monomorphism. This implies trivially that $\alpha$ is invertible. If $1_{E(W)}-\rho$ is invertible, then $\mu \circ\left(1_{E(W)}-\rho\right)=\left(1_{W}-e\right) \circ \mu$ and then trivially $1_{W}-e$ is a pure-monomorphism, which splits by the above argument. Clearly this implies that $e=0$. Hence $\alpha^{\prime} \circ \alpha=0 \Rightarrow \alpha=0$ and this is impossible, since by hypothesis $\alpha \neq 0$. We deduce that if $\beta$ is invertible, then so is $\alpha$. Similarly if $1_{E(W)}-\beta$ is invertible, then so is $1_{W}-\alpha$. We infer that $\operatorname{End}_{\mathcal{C}}(W)$ is local. 
(i) $\Rightarrow$ (ii) Let $\operatorname{End}(W) \cong \operatorname{End}(\mathrm{H}(W))$ be local and let $\mu: W \rightarrow E(W)$ be the pure-injective envelope of $W$. Since $\mathrm{H}(W)$ is injective in the Frobenius category mod- $\mathcal{C}^{\mathrm{b}}$, it follows that $\mathrm{H}(W)$ is a uniform object in mod- $\mathcal{C}^{\mathrm{b}}$, hence any of its non-zero finitely presented subobjects is indecomposable. Let $\alpha: M \hookrightarrow \mathrm{H}(W)$ be a non-zero subobject in Mod- $\mathcal{C}^{\mathrm{b}}$, and assume that $M=M_{1} \oplus M_{2}$. If both $M_{1}, M_{2}$ are non-zero, then there are non-zero morphisms $\rho_{i}: \mathrm{H}\left(X_{i}\right) \rightarrow M_{i}$, where the $X_{i}$ are compact. Since each $F_{i}=\operatorname{Im}\left(\rho_{i} \circ \alpha\right)$ is finitely presented, $F_{1} \oplus F_{2}$ is a finitely presented subobject of $\mathrm{H}(W)$. Since $\mathrm{H}(W)$ is uniform in mod- $\mathcal{C}^{\mathrm{b}}$, it follows that $F_{1} \oplus F_{2}$ is indecomposable, hence $F_{1}=0$ or $F_{2}=0$ and this is impossible. Hence $M_{1}=0$ or $M_{2}=0$ and $M$ is indecomposable. This implies that $\mathrm{H}(W)$ is uniform in Mod- $\mathcal{C}^{\mathrm{b}}$ and therefore its injective envelope $\mathrm{H}(E(W))$ is indecomposable. Hence the ring $\operatorname{End}(E(W)) \cong \operatorname{End}(\mathrm{H}(E(W)))$ is local.

COROLLARY 8.2. Let $X$ be a compact object with local endomorphism ring. If $Y$ is compact, then $\mathrm{E}(X) \cong \mathrm{E}(Y)$ implies that $X \cong Y$. In particular the operation $\mathrm{E}$ of taking pure-injective envelopes of compact objects, induces an injective function

$$
\mathrm{E}: \operatorname{lnd}\left(\mathcal{C}^{\mathrm{b}}\right) \longrightarrow \mathrm{Zg}(\mathcal{C}), X \mapsto \mathrm{E}(X)
$$

Proof. Let $\mu: X \rightarrow E$ and $\kappa: Y \rightarrow E$ be pure-injective envelopes. By Lemma 8.1 both rings $\operatorname{End}(Y)$ and $\operatorname{End}(E)$ are local, hence $\mathrm{H}(E)$ is an indecomposable injective functor, and the inclusions $\mathrm{H}(\kappa): \mathrm{H}(X) \hookrightarrow \mathrm{H}(E)$ and $\mathrm{H}(\lambda): \mathrm{H}(Y) \hookrightarrow \mathrm{H}(E)$ are injective envelopes. In particular $F:=$ $\mathrm{H}(X) \cap \mathrm{H}(Y) \neq 0$. Let $Z$ be a compact object such that $F(Z) \neq 0$, and let $\rho: \mathrm{H}(Z) \rightarrow F$ be a non-zero morphism. Then the image $G$ of the composition $\rho \circ \mathrm{H}(\kappa): \mathrm{H}(Z) \rightarrow \mathrm{H}(X)$ is a finitely presented functor, and we have an inclusion $\xi: G \hookrightarrow F$ which induces inclusions $\xi \circ \alpha: G \hookrightarrow \mathrm{H}(X)$ and $\xi \circ \beta: G \hookrightarrow \mathrm{H}(Y)$. Since the category mod- $\mathcal{C}^{\mathrm{b}}$ is Frobenius, the projective objects $\mathrm{H}(X)$ and $\mathrm{H}(Y)$ in mod- $\mathcal{C}^{\mathrm{b}}$ are injective. Hence the inclusions $\xi \circ \alpha$ and $\xi \circ \beta$ are injective envelopes of $G$ in mod- $\mathcal{C}^{\mathrm{b}}$. Then $\mathrm{H}(X) \cong \mathrm{H}(Y)$, hence $X \cong Y$.

\subsection{EXISTENCE OF RIGHT AR-TRIANGLES IN $\mathcal{C}^{b}$}

The following basic result, which generalizes a ring theoretic result of W. Zimmermann [81, Theorem 1], see also [57] for a recent related treatment, gives necessary and sufficient conditions for the existence of an AR-triangle in $\mathcal{C}^{\mathrm{b}}$ starting at a compact object. 
THEOREM 8.3. For a compact object $X$ in $\mathcal{C}$ the following are equivalent.

(i) There exists an AR-triangle $W \rightarrow Z \rightarrow X \rightarrow \Sigma(W)$ in $\mathcal{C}^{b}$.

(ii) The object $X$ has local endomorphism ring and the $\mathrm{m}$-dual object $\mathbb{D}_{\mathfrak{m}}(X)$ of $X$ is the pure-injective envelope of a compact object.

(iii) $X$ has local endomorphism ring, the simple functor $S_{X, \mathfrak{m}}$ is finitely presented, and the subfunctor $\mathrm{H}(X)_{\mathrm{m}} \hookrightarrow \mathrm{H}(X)$ admits a projective cover.

Proof. (i) $\Rightarrow$ (ii) Let $W \stackrel{\alpha}{\rightarrow} Z \stackrel{\beta}{\rightarrow} X \stackrel{\gamma}{\rightarrow} \Sigma(W)$ be an AR-triangle in $\mathcal{C}^{\text {b }}$ and let $\Sigma^{-1} \mathbb{D}_{\mathfrak{m}}(X) \stackrel{g}{\rightarrow} A \stackrel{f}{\rightarrow} X \stackrel{h}{\rightarrow} \mathbb{D}_{\mathfrak{m}}(X)$ be the AR-triangle in $\mathcal{C}$ ending at $X$. Since $\beta$ is not split epic and $f$ is right almost split, there exists a morphism of triangles:

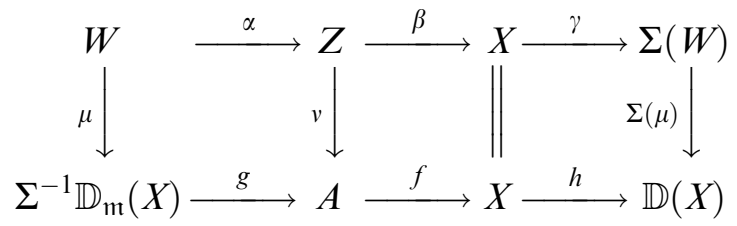

Let $W \stackrel{\mu}{\rightarrow} \Sigma^{-1} \mathbb{D}_{\mathrm{m}}(X) \stackrel{\kappa}{\rightarrow} B \stackrel{\xi}{\rightarrow} \Sigma(W)$ be a triangle in $\mathcal{C}$. We show that the morphism $\xi$ is phantom. Let $T$ be an arbitary compact object and let $\rho: T \rightarrow B$ be any morphism. By the Octahedral Axiom, there exists a morphism of triangles:

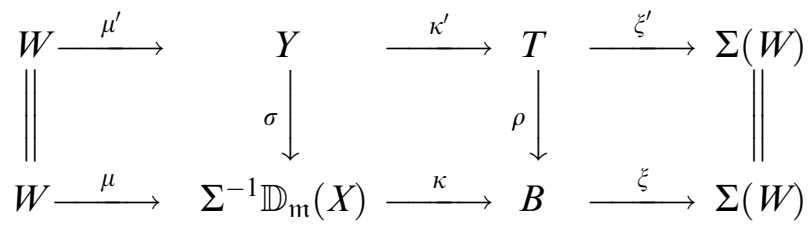

Since the objects $W$ and $T$ are compact, so is $Y$. If $\mu^{\prime}$ is a split monic, then $\xi^{\prime}=0$. It follows that $\rho \circ \xi=0$ and this implies that $\rho$ factors through $\kappa$. Assume now that $\mu^{\prime}$ is not a split monic. Since $\alpha$ is left almost split, $\mu^{\prime}$ factors through $\alpha$. Hence there exists $\phi: Z \rightarrow Y$ such that $\mu^{\prime}=\alpha \circ \phi$. Then $\mu^{\prime}=\alpha \circ \phi \Rightarrow \mu^{\prime} \circ \sigma=\alpha \circ \phi \circ \sigma \Rightarrow \mu=\alpha \circ \phi \circ \sigma \Rightarrow \mu \circ g=\alpha \circ \phi \circ \sigma \circ g \Rightarrow$ $\alpha \circ v=\alpha \circ \phi \circ \sigma \circ g$. Hence $v-\phi \circ \sigma \circ g$ factors through $\beta$ and therefore $v-\phi \circ \sigma \circ g=\beta \circ \psi$ for some map $\psi: X \rightarrow A$. Then $v \circ f-\phi \circ \sigma \circ g \circ f=$ $\beta \circ \psi \circ f \Rightarrow \beta=\beta \circ \psi \circ f$. Since $\operatorname{End}_{\mathcal{C}}(X)$ is local, $\psi \circ f$ is invertible or $1_{X}-\psi \circ f$ is invertible. However $\psi \circ f$ cannot be invertible, since $f$ is right almost split. Hence $1_{X}-\psi \circ f$ is invertible. Then $\beta=0$, hence $\gamma$ is a split monic and therefore invertible since $\operatorname{End}(X)$ and $\operatorname{End}(W)$ are local. Consequently $Z=0$ and the morphism $\Sigma(W) \rightarrow 0$ is left almost split. Since $\mu^{\prime}$ is not a split monomorphism, the same is true for $\Sigma\left(\mu^{\prime}\right): \Sigma(W) \rightarrow \Sigma(Y)$. Hence $\Sigma\left(\mu^{\prime}\right)$ factors through $\Sigma(W) \rightarrow 0$, i.e. $\mu^{\prime}=0$. Then diagram (1) shows that $h=0$ and this is impossible, since by construction we always have $h \neq 0$. 
Hence $\mu^{\prime}$ is a split monomorphism. Then $\xi^{\prime}=0$ and from diagram (2) we have $\rho \circ \xi=0$. Since $T$ was an arbitrary compact object, this shows that $\xi$ is pure-phantom. Equivalently $\mu$ is pure-mono and then so is $\Sigma^{-1}(\mu)$. Since $\mathbb{D}_{\mathfrak{m}}(X)$ has local endomorphism ring, it follows that $\Sigma^{-1}(\mu)$ : $\Sigma^{-1}(W) \rightarrow \mathbb{D}_{\mathfrak{m}}(X)$ is a pure-injective envelope.

(ii) $\Rightarrow$ (iii) Let $Z$ be compact and let $\kappa: \mathrm{H}(Z) \longmapsto \mathrm{H}\left(\mathbb{D}_{\mathfrak{m}}(X)\right)$ be its injective envelope. Then $\operatorname{End}(Z)$ is local by Lemma 8.1. Since $\mu: S_{X, \mathfrak{m}} \longmapsto \mathrm{H}\left(\mathbb{D}_{\mathfrak{m}}(X)\right)$ is an injective envelope, it follows that $S_{X, \mathfrak{m}} \cap \mathrm{H}(Z) \neq 0$, hence we have an inclusion $\lambda: S_{X, \mathrm{~m}} \hookrightarrow \mathrm{H}(Z)$. Let $\gamma: X \rightarrow Z$ be the unique morphism in $\mathcal{C}^{\text {b }}$ such that $\mathrm{H}(h)=\varepsilon \circ \lambda$ and let $(T): \Sigma^{-1}(Z) \rightarrow Y \stackrel{\beta}{\rightarrow} X \stackrel{\gamma}{\rightarrow} Z$ be a triangle in $\mathcal{C}^{\text {b }}$. Then $S_{X, m}=\operatorname{Im}(\mathrm{H}(\gamma))$ is finitely presented and therefore $\beta$ is right almost split and $\operatorname{ImH}(\beta)=\mathrm{H}(X)_{\mathfrak{m}}$. Since $\operatorname{End}(Z)$ is local, $(T)$ is an AR-triangle in $\mathcal{C}^{\mathrm{b}}$, hence $f$ is right minimal. Then the projection $\mathrm{H}(Y) \rightarrow \mathrm{H}(X)_{\mathfrak{m}}$ is a projective cover.

(iii) $\Rightarrow$ (i) We have a minimal projective presentation $\mathrm{H}(Y) \rightarrow \mathrm{H}(X) \rightarrow$ $S_{X, \mathrm{~m}} \rightarrow 0$ in mod- $\mathcal{C}^{\mathrm{b}}$ and a triangle $(T): W \rightarrow Y \rightarrow X \rightarrow \Sigma W$ in $\mathcal{C}^{\mathrm{b}}$. By the minimality of the presentation, $Y \rightarrow X$ is right minimal, hence minimal right almost split, since $S_{X, \mathfrak{m}}$ is simple and $\operatorname{End}(X)$ is local. So $(T)$ is an ARtriangle in $\mathcal{C}^{\mathrm{b}}$.

The above result suggests the following definition.

DEFINITION 8.4. The finite spectrum $\operatorname{Fin}(\mathcal{C})$ of $\mathcal{C}$ is the set of pureinjective envelopes of the isoclasses of compact objects with local endomorphism ring:

$$
\operatorname{Fin}(\mathcal{C}):=\left\{\mathrm{E}(X) \mid X \in \operatorname{Ind}\left(\mathcal{C}^{b}\right)\right\} .
$$

We call the elements of $\operatorname{Fin}(\mathcal{C})$ finite points of $\mathcal{C}$ or $\operatorname{Zg}(\mathcal{C})$. Then Lemma 8.1 ensures that any finite point is a point of $\operatorname{Zg}(\mathcal{C})$, i.e. $\operatorname{Fin}(\mathcal{C}) \subseteq \operatorname{Zg}(\mathcal{C})$. The following result, observed independently by Garkusha-Prest [32], shows that if $\mathcal{C}^{\mathrm{b}}$ is Krull-Schmidt then we have a good supply of finite points. We refer the reader to Proposition 5.4 of Herzog's paper [41] for the module theoretic analogue.

PROPOSITION 8.5. If $\mathcal{C}^{\mathrm{b}}$ is a Krull-Schmidt category, then $\operatorname{Fin}(\mathcal{C})$ is a dense subset of $\mathrm{Zg}(\mathcal{C})$. In particular $\mathrm{Fin}(\mathcal{C})$ contains all isolated points.

Proof. Let $\mathbf{U}_{\Phi}$ be a closed subset such that $\operatorname{Fin}(\mathcal{C}) \subseteq \mathbf{U}_{\Phi}$ and let $\phi: Y \rightarrow Z$ be any morphism in $\Phi$. Consider the exact sequence $0 \rightarrow F \stackrel{\kappa}{\rightarrow} \mathrm{H}(Y) \stackrel{\mathrm{H}(\phi)}{\rightarrow} \mathrm{H}(Z)$. Since any finite point is $\phi$-injective, it follows that $[F, \mathrm{H}(E(X))]=0$, for any indecomposable compact object $X$. If $Y=\oplus_{i=1}^{n} Y_{i}$ is an indecomposable decomposition of $Y$, then $Y \hookrightarrow \oplus_{i=1}^{n} E\left(Y_{i}\right)$ is a pure-injective envelope and $\mathrm{H}(Y) \hookrightarrow \oplus_{i=1}^{n} \mathrm{H}\left(E\left(Y_{i}\right)\right)$ is an injective envelope. Hence $\left[F, \oplus_{i=1}^{n} \mathrm{H}\left(E\left(Y_{i}\right)\right)\right]=0$ and consequently $F=0$. This implies that $\phi$ is a split monomorphism. 
Therefore $\Phi$ consists of split monomorphisms and consequently $\mathbf{U}_{\Phi}=\operatorname{Zg}(\mathcal{C})$. Hence $\operatorname{Fin}(\mathcal{C})$ is dense subset of $\operatorname{Zg}(\mathcal{C})$.

Now we can prove the following basic result.

THEOREM 8.6. The following conditions are equivalent.

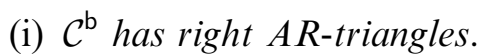

(ii) $\operatorname{Max}_{\text {Ind }}(\mathcal{C}) \subseteq \operatorname{Fin}(\mathcal{C})$.

(iii) $\operatorname{Max}_{\operatorname{lnd}}(\mathcal{C}) \subseteq \operatorname{Isol}(\mathcal{C})$.

If $\mathcal{C}^{\mathrm{b}}$ is a Krull-Schmidt category with right AR-triangles, then:

$$
\operatorname{Max}_{\text {Ind }}(\mathcal{C})=\operatorname{Max}(\mathcal{C})=\operatorname{Isol}(\mathcal{C}) \subseteq \operatorname{Fin}(\mathcal{C}) .
$$

Proof. That (i) is equivalent to (ii) is a direct consequence of Theorem 8.3.

(i) $\Rightarrow$ (iii) Let $\mathbb{D}_{\mathfrak{m}}(X)$ be a maximal point, where $X$ has local endomorphism ring. Since $\mathcal{C}^{\mathrm{b}}$ has right AR-triangles, by Theorem 8.3 the simple functor $S_{X, \mathrm{~m}}$ is finitely presented. Then by Corollary 7.8 we have that $\mathbb{D}_{\mathfrak{m}}(X)$ is isolated.

(iii) $\Rightarrow$ (ii) Let $X$ be a compact object with local endomorphism ring. By hypothesis, $\Sigma^{-1} \mathbb{D}_{\mathfrak{m}}(X)$ is isolated. Hence there exists a morphism $\alpha: Z \rightarrow Y$ between compact objects, such that any morphism $Z \rightarrow E$ factors through $\alpha$ for any point $E \in \operatorname{Zg}(\mathcal{C}) \backslash\left\{\Sigma^{-1} \mathbb{D}_{\mathfrak{m}}(X)\right\}$, and there exists a morphism $\beta: Z \rightarrow \Sigma^{-1} \mathbb{D}_{\mathfrak{m}}(X)$ which does not factor through $\alpha$. Let $\mu: Z \rightarrow E(Z)$ be the pure-injective envelope of $Z$. If $E(Z) \neq \Sigma^{-1} \mathbb{D}_{\mathfrak{m}}(X)$, then by construction there exists a morphism $\rho: Y \rightarrow E(Z)$ such that $\alpha \circ \rho=\mu$. Since $\mu$ is puremono and $\Sigma^{-1} \mathbb{D}_{\mathfrak{m}}(X)$ is pure-injective, there exists a morphism $\gamma: E(Z) \rightarrow$ $\Sigma^{-1} \mathbb{D}_{\mathfrak{m}}(X)$ such that $\mu \circ \gamma=\beta$, hence $\alpha \circ \rho \circ \gamma=\beta$ and this is impossible by the construction of $\beta$. Hence $\Sigma^{-1} \mathbb{D}_{\mathfrak{m}}(X) \cong E(Z)$ is the pure-injective envelope of the compact object $Z$.

The last assertion follows from Lemma 7.5 and Corollary 7.7.

\subsection{EXISTENCE OF LEFT ALMOST SPLIT MORPHISMS AND LEFT AUSLANDER-REITEN TRIANGLES IN $\mathcal{C}^{\mathrm{b}}$}

Now we turn our attention to the investigation of the existence of left almost split morphisms and AR-triangles in $\mathcal{C}^{\mathrm{b}}$ starting at compact objects, in connection with the Ziegler spectrum and the injective envelopes of finitely presented simple functors. We begin our analysis with the following result.

PROPOSITION 8.7. For a compact object $W$, the following are equivalent:

(i) $W$ is the source of a (minimal) left almost split morphism in $\mathcal{C}^{b}$.

(ii) $\mathrm{H}(W)$ is the injective envelope of a simple object $S$ in mod-C $\mathcal{C}^{\mathrm{b}}$ (and $S$ admits a projective cover). 
In particular $W$ is the source of an AR-triangle in $\mathcal{C}^{\mathrm{b}}$ iff $\mathrm{H}(W)$ it is the injective envelope of a simple object in mod- $\mathcal{C}^{\mathrm{b}}$ which admits a projective cover.

Proof. (i) $\Rightarrow$ (ii) Let $\alpha: W \rightarrow Y$ be a left almost split morphism in $\mathcal{C}^{\mathrm{b}}$ and consider the exact sequence $0 \rightarrow S \stackrel{\mu}{\rightarrow} \mathrm{H}(W) \rightarrow \mathrm{H}(Y)$ in mod- $\mathcal{C}^{\mathrm{b}}$. Then $S$ is finitely presented and it suffices to show that $S$ is a simple in the Frobenius category mod- $\mathcal{C}^{\mathrm{b}}$, since $\mathrm{H}(W)$ is an injective object with local endomorphism ring. If $S=0$, then $\mathrm{H}(\alpha)$ is monic, which splits since $\mathrm{H}(W)$ is injective in mod$\mathcal{C}^{b}$. Then $\alpha$ is a split monic and this is not true, since $\alpha$ is left almost split. Hence $S \neq 0$. Let $\omega: S \rightarrow F$ be a morphism in mod- $\mathcal{C}^{\mathrm{b}}$. Then there exists a monomorphism $\kappa: F \longmapsto \mathrm{H}(X)$ with $X$ in $\mathcal{C}^{\mathrm{b}}$. Since $\mathrm{H}(X)$ is injective, there exists $\mathrm{H}(\rho): \mathrm{H}(Y) \rightarrow \mathrm{H}(X)$ such that $\mu \circ \mathrm{H}(\rho)=\omega \circ \kappa$. If $\rho$ is not split mono, then $\rho$ factors through $\alpha$ and this implies that $\omega=0$. If $\rho$ is split monic, then $\omega$ is a monic. This shows that $S$ is a simple object in mod- $\mathcal{C}^{b}$. Now if $\alpha$ is minimal, it follows by Lemma 3.2 that $W \stackrel{\alpha}{\rightarrow} Y \rightarrow X \rightarrow \Sigma(W)$ is an ARtriangle in $\mathcal{C}^{\mathrm{b}}$. Since $S$ is a factor of $\mathrm{H}\left(\Sigma^{-1}(X)\right)$ and $X$ has local endomorphism ring, we infer that $S$ admits a projective cover.

(ii) $\Rightarrow$ (i) Let $\mathrm{H}(Z) \rightarrow \mathrm{H}(X) \stackrel{\epsilon}{\rightarrow} S \rightarrow 0$ be a finite presentation of a finitely presented simple functor $S$ and let $\kappa: S \longmapsto \mathrm{H}(W)$ be an injective envelope. Then $\epsilon \circ \kappa: \mathrm{H}(X) \rightarrow \mathrm{H}(W)$ is of the form $\mathrm{H}(\gamma)$, where $\gamma: X \rightarrow W$ is a morphism in $\mathcal{C}^{\mathrm{b}}$. If $X \stackrel{\gamma}{\rightarrow} W \stackrel{\alpha}{\rightarrow} Y \rightarrow \Sigma(X)$ is a triangle in $\mathcal{C}^{\mathrm{b}}$, then $\alpha$ is not a split monomorphism since $\operatorname{KerH}(\alpha)=S$. Let $\rho: W \rightarrow T$ be a morphism in $\mathcal{C}^{\text {b }}$. Since $S$ is simple, the composition $\kappa \circ \mathrm{H}(\rho)$ is zero or a monomorphism. If $\kappa \circ \mathrm{H}(\rho)$ is a monomorphism, then $\mathrm{H}(\rho)$ is a monomorphism, since $\kappa$ is essential. This implies that $\rho$ is a split monomorphism, since $\mathrm{H}(W)$ is injective. Hence if $\rho$ is not a split monomorphism, then $\kappa \circ \mathrm{H}(\rho)=0 \Rightarrow$ $\mathrm{H}(\gamma) \circ \mathrm{H}(\rho)=0 \Rightarrow \gamma \circ \rho=0 \Rightarrow \rho$ factors through $\alpha$. This shows that $\alpha$ is a left almost split. If $\varepsilon: \mathrm{H}(X) \rightarrow S$ is a projective cover, then $\operatorname{End}(X)$ is local and therefore $W \stackrel{\alpha}{\rightarrow} Y \rightarrow \Sigma(X) \rightarrow \Sigma(W)$ is an AR-triangle in $\mathcal{C}^{\mathrm{b}}$.

Now we can prove the following result which characterizes when a compact object occurs as a source of a left almost split morphism or an AR-triangle in $\mathcal{C}^{\mathrm{b}}$ and generalizes a module theoretic result of Krause, see [53, Theorem 3.6].

THEOREM 8.8. Let $\mathrm{E}(W)$ be a finite point, that is, $\mathrm{E}(W)$ is the pure-injective envelope of a non-zero compact object $W$. Then the following are equivalent.

(i) $E(W)$ is the source of a (minimal) left almost split morphism in $\mathcal{C}$.

(ii) $W$ is the source of a (minimal) left almost split morphism in $\mathcal{C}^{b}$.

If (i) holds, then $E(W)$ is isolated in $\operatorname{Zg}(\mathcal{C})$. In particular $W$ is the source of an $A R$-triangle in $\mathcal{C}^{\mathrm{b}}$ iff $E(W)$ is the source of an AR-triangle in $\mathcal{C}$.

Proof. (i) $\Rightarrow$ (ii) By Theorems 7.1 and 8.3, $E(W)$ is isomorphic to some m-dual object $\Sigma^{-1}\left(\mathbb{D}_{\mathfrak{m}}(X)\right)$ and there exists a morphism of triangles 


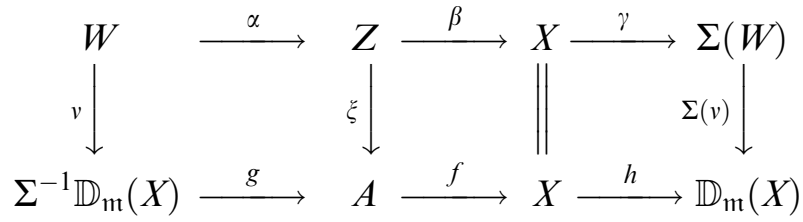

where $\mu$ is a pure-injective enveloped and $g$ is left almost split in $\mathcal{C}$. Since $\operatorname{ImH}\left(\Sigma^{-1}(h)\right)$ is the simple functor $S_{\Sigma^{-1} X, \mathfrak{m}}$ and $\mathrm{H}(\mu)$ is a monic, we have $S_{\Sigma^{-1} X, \mathrm{~m}} \cap \mathrm{H}(W) \neq 0$ and therefore there exists an inclusion $\kappa: S_{\Sigma^{-1} X, \mathfrak{m}} \longmapsto$ $\mathrm{H}(W)$ such that $\kappa \circ \mathrm{H}(v)=\mu$ where $\mu$ is the inclusion $S_{\Sigma^{-1} X, \mathrm{~m}} \hookrightarrow$ $\mathrm{H}\left(\Sigma^{-1} \mathbb{D}_{\mathfrak{m}}(X)\right)$. This implies that $S_{\Sigma^{-1} X, \mathfrak{m}}=\mathrm{H}\left(\Sigma^{-1}(\gamma)\right)$ and therefore $S_{\Sigma^{-1} X, \mathfrak{m}}$ is finitely presented. Hence $\mathrm{H}(W)$ is the injective envelope of a simple functor in mod- $\mathcal{C}^{\mathrm{b}}$ and therefore by Proposition 8.7, $W$ is the source of a left almost split morphism in $\mathcal{C}^{\mathrm{b}}$. Now if $g$ is in addition left minimal, the lower triangle above is an AR-triangle and therefore $\operatorname{End}(X)$ is local. Then clearly the upper triangle is an AR-triangle in $\mathcal{C}^{\mathrm{b}}$ and $\alpha$ is minimal left almost split.

(ii) $\Rightarrow$ (i) By Proposition 8.7, there exists a simple object $S$ in mod- $\mathcal{C}^{b}$ and an injective envelope $\kappa: S \longmapsto \mathrm{H}(W)$ in mod- $\mathcal{C}^{\mathrm{b}}$. Consider the pure-injective envelope $\lambda: W \rightarrow E(W)$ in $\mathcal{C}$. Since $\mathrm{H}(\lambda): \mathrm{H}(W) \longmapsto \mathrm{H}(E(W))$ is an injective envelope, there exists a morphism $v: S \rightarrow \mathrm{H}(W)$ such that $v \circ \mathrm{H}(\lambda)=\kappa$, which is a monomorphism since $S$ is simple in mod- $\mathcal{C}^{\mathrm{b}}$. Let $\alpha: S \rightarrow M$ be a non-zero morphism in Mod- $\mathcal{C}^{\mathrm{b}}$ and let $\xi: F \rightarrow S$ be its kernel. If $F \neq 0$, then there exists a non-zero morphism $\beta: \mathrm{H}(T) \rightarrow F$ for some compact $T$. Then the map $\beta \circ \xi: \mathrm{H}(T) \rightarrow S$ is zero or an epic. Both cases are impossible since both $\beta$ and $\alpha$ are non-zero. Hence any non-zero morphism $\alpha: S \rightarrow M$ in Mod- $\mathcal{C}^{\mathrm{b}}$ is zero or a monic and therefore $S$ is simple in Mod- $\mathcal{C}^{\mathrm{b}}$. Then its injective envelope is of the form $\mathrm{H}\left(\mathbb{D}_{\mathfrak{m}}(X)\right)$ for some compact $X$. Hence $\mathrm{H}(E(W)) \cong \mathrm{H}\left(\mathbb{D}_{\mathfrak{m}}(X)\right)$ or equivalently $E(W) \cong \mathbb{D}_{\mathfrak{m}}(X)$. Hence the finite point $E(W)$ is maximal and then, by Theorem 7.1, $E(W)$ is the source of a left almost split morphism in $\mathcal{C}$. Finally if $W$ is the source of a minimal left almost split morphism in $\mathcal{C}^{\mathrm{b}}$, then diagram (1) of Theorem 8.3 shows that $E(W)$ is the source of a minimal left almost split morphism in $\mathcal{C}$.

Assume now that (i) holds. Then as before we have the diagram of triangles $(*)$, where $\alpha$, resp. $g$, is a left almost split morphism in $\mathcal{C}^{\mathrm{b}}$, resp. $\mathcal{C}$, and $v$ is a pure-injective envelope. Consider the Ziegler-closed set $\mathbf{U}_{\alpha}$ and observe that $E$ is not in $\mathbf{U}_{\alpha}$, since otherwise $\Sigma^{-1}(h)=\Sigma^{-1}(\gamma) \circ v=0$ and this is not the case. Let $M \in \operatorname{Zg}(\mathcal{C}) \backslash\{E\}$ and let $\phi: W \rightarrow M$ be any morphism. Since $M$ is pure-injective and $v$ is a pure-injective envelope, there exists $\psi: E \rightarrow M$ such that $v \circ \psi=\phi$. Since $M$ is not isomorphic to $E$, $\psi$ is not a split monic. Since $g$ is left almost split, there exists $\chi: A \rightarrow M$ such that $g \circ \chi=\psi$. Then $v \circ g \circ \chi=\Sigma^{-1}(\gamma) \circ v \circ \chi=\phi$. Hence $M \in \mathbf{U}_{\alpha}$ and then $\operatorname{Zg}(\mathcal{C}) \backslash\{E\}=\mathbf{U}_{\alpha}$. It follows that $\{E\}$ is open in the Ziegler topology, and therefore $E$ is an isolated point in $\mathrm{Zg}(\mathcal{C})$. 
From Corollary 7.7 and Theorem 8.8, we deduce the following consequence.

COROLLARY 8.9. Let $E(W)$ a finite point in $\operatorname{Zg}(\mathcal{C})$, that is, $E(W)$ is the pure-injective envelope of a non-zero compact object $W$ with local endomorphism ring. Then the following are equivalent:

(i) $E(W)$ is isolated in $\mathrm{Zg}(\mathcal{C})$.

(ii) $E(W) \in \operatorname{Max}(\mathcal{C})$.

(iii) $E(W)$ is the source of a left almost split morphism in $\mathcal{C}$.

(iv) $W$ is the source of a left almost split morphism in $\mathcal{C}^{b}$.

Summarizing what we proved so far in this subsection we have the following characterization of when a given compact object is the source of an AR-triangle in $\mathcal{C}^{\mathrm{b}}$ and when $\mathcal{C}^{\mathrm{b}}$ has left AR-triangles.

THEOREM 8.10. If $W$ is a compact object, then the following are equivalent.

(i) There exists an AR-triangle $W \rightarrow Y \rightarrow X \rightarrow \Sigma W$ in $\mathcal{C}^{b}$.

(ii) The functor $\mathrm{H}(W)$ is an essential extension of a simple functor $S$ and $S$ admits a projective cover.

(iii) The pure-injective envelope of $W$ is the $\mathrm{m}$-dual object $\mathbb{D}_{\mathfrak{m}}(X)$ of a compact object $X$ with local endomorphism ring.

In particular $\mathcal{C}^{\mathrm{b}}$ has left $A R$-triangles iff $\operatorname{Fin}(\mathcal{C}) \subseteq \operatorname{Max}_{\operatorname{lnd}}(\mathcal{C})$.

COROLLARY 8.11. If $\mathcal{C}^{\mathrm{b}}$ is Krull-Schmidt, then the following are equivalent.

(i) $\mathcal{C}^{\text {b }}$ has left AR-triangles.

(ii) $\operatorname{Fin}(\mathcal{C}) \subseteq \operatorname{Max}(\mathcal{C})$.

(iii) $\operatorname{Fin}(\mathcal{C}) \subseteq \operatorname{Isol}(\mathcal{C})$.

If (i) holds, then: $\operatorname{Fin}(\mathcal{C})=\operatorname{Isol}(\mathcal{C}) \subseteq \operatorname{Max}_{\operatorname{lnd}}(\mathcal{C})=\operatorname{Max}(\mathcal{C})$.

Combining Theorem 8.6 and Corollary 8.11 we have the following result which gives a characterization of the existence of AR-triangles in $\mathcal{C}^{b}$ in terms of properties of m-dual objects of compact objects and the finite/maximal spectrum.

THEOREM 8.12. The following conditions are equivalent.

(i) $\mathcal{C}^{\mathrm{b}}$ has AR-triangles.

(ii) $\operatorname{Fin}(\mathcal{C})=\operatorname{Max}_{\operatorname{lnd}}(\mathcal{C})$.

(iii) The Auslander-Reiten operator $\tau^{+}: \operatorname{Ind}\left(\mathcal{C}^{\mathrm{b}}\right) \rightarrow \operatorname{Max}_{\operatorname{lnd}}(\mathcal{C})$ given by $X \mapsto \tau^{+}(X)=\Sigma^{-1} \mathbb{D}_{\mathfrak{m}}(X)$ induces a bijection $\tau^{+}: \operatorname{Ind}\left(\mathcal{C}^{\mathrm{b}}\right) \rightarrow \operatorname{Fin}(\mathcal{C})$. 
If (i) holds, then we have equalities: $\operatorname{Fin}(\mathcal{C})=\operatorname{Max}_{\operatorname{Ind}}(\mathcal{C})=\operatorname{Isol}(\mathcal{C})$. If $\mathcal{C}^{\mathrm{b}}$ is a Krull-Schmidt category, then $\mathcal{C}^{\mathrm{b}}$ has AR-triangles if and only if $\operatorname{Fin}(\mathcal{C})=\operatorname{Max}(\mathcal{C})$.

Remark 8.13. Let $\mathrm{Ho}(\mathcal{S} p)$ be the stable homotopy of spectra. Since $\mathrm{Ho}(\mathcal{S} p)$ is compactly self-dual [61, Theorem 1.19], it follows that the full subcategory $\mathrm{Ho}(\mathcal{S} p)^{\mathrm{b}}$ of finite spectra has right AR-triangles iff $\mathrm{Ho}(\mathcal{S} p)^{\mathrm{b}}$ has left AR-triangles. It seems to be an interesting problem to characterize the finite ( $p$-local) spectra with local endomorphism ring which occur as a source or target of an AR-triangle in $\operatorname{Ho}(\mathcal{S} p)^{\mathrm{b}}$. Note that the finite $p$-local spectra form a Krull-Schmidt category. This problem is also related to the validity of Freyd's generating hypothesis (FGH) in stable homotopy theory: if $(\mathrm{FGH})$ holds, then $\operatorname{Max}(\mathrm{Ho}(\mathcal{S} p)) \nsubseteq \mathrm{Fin}(\mathrm{Ho}(\mathcal{S} p))$, see [52].

\section{Compact Pure-Injective Objects, Derived categories and Gorenstein Algebras}

Throughout we fix a compactly generated triangulated category $\mathcal{C}$. In this section we study when a compact object in $\mathcal{C}$ is pure-injective. This is related to the question of when an AR-triangle in the subcategory of compact objects remains an AR-triangle in $\mathcal{C}$. We present a variety of examples satisfying this condition concentrating on derived categories where existence of AR-triangles for perfect complexes, resp. bounded complexes of finitely generated modules, is related to Gorensteinness, resp. non-singularity. In this way we give simple proofs of generalizations of well-known results of Happel [37, 38], see also the recent work of Krause [57]. Finally we give methods for constructing new Gorenstein algebras from old ones.

\subsection{WHEN COMPACT OBJECTS ARE PURE-INJECTIVE}

From the results of the previous section it follows that AR-triangles in $\mathcal{C}^{\mathrm{b}}$ behave nicely when compact objects are pure-injective. This is related to the question of when AR-triangles in $\mathcal{C}^{\mathrm{b}}$ remain such in $\mathcal{C}$. We begin our analysis of when compact are pure-injective objects with the following preliminary results.

LEMMA 9.1. Let $(T): W \rightarrow Z \rightarrow X \rightarrow \Sigma(W)$ be an AR-triangle in $\mathcal{C}^{\text {b }}$. Then the following are equivalent:

(i) $(T)$ is an AR-triangle in $\mathcal{C}$.

(ii) $W$ is pure-injective.

(iii) The (pure mono-)morphism $W \rightarrow \Sigma^{-1} \mathbb{D}_{\mathfrak{m}}(X)$ is invertible. 
Proof. Since $X$ is compact, the implication (i) $\Rightarrow$ (ii) follows from Lemma 6.4. If (ii) holds and $(T)$ is an AR-triangle in $\mathcal{C}^{\mathrm{b}}$, from the proof of Theorem 8.3 it follows that the canonically constructed morphism $\mu: W \rightarrow \Sigma^{-1} \mathbb{D}_{\mathfrak{m}}(X)$ is a pure-injective envelope. Then $\mu$ is invertible, since $W$ is pure-injective. Finally if (iii) holds, then since an AR-triangle in $\mathcal{C}$ is uniquely determined up to isomorphism by its end terms, it follows that the AR-triangle $(T)$ in $\mathcal{C}^{\mathrm{b}}$ is isomorphic to $\Sigma^{-1} \mathbb{D}_{\mathfrak{m}}(X) \rightarrow Z \rightarrow X \rightarrow \mathbb{D}_{\mathfrak{m}}(X)$ which is an AR-triangle in $\mathcal{C}$.

COROLLARY 9.2. Consider the following statements:

(i) Any AR-triangle in $\mathcal{C}^{\text {b }}$ is an AR-triangle in $\mathcal{C}$.

(ii) Any compact object in $\mathcal{C}$ with local endomorphism ring is pure-injective.

Then (ii) $\Rightarrow$ (i). If $\mathcal{C}^{b}$ has left AR-triangles, then (ii) and (i) are equivalent. In particular if $\mathcal{C}^{\mathrm{b}}$ is a Krull-Schmidt category with left AR-triangles, then any compact object is pure-injective iff any AR-triangle in $\mathcal{C}^{\mathrm{b}}$ is an AR-triangle in $\mathcal{C}$.

Proof. Assume that (ii) holds and let $(T): W \stackrel{\alpha}{\rightarrow} Z \stackrel{\beta}{\rightarrow} X \stackrel{\gamma}{\rightarrow} \Sigma(W)$ be an AR-triangle in $\mathcal{C}^{\mathrm{b}}$. Since $W$ is compact with local endomorphism ring, by hypothesis $W$ is pure-injective, hence by Lemma 9.1 we infer that $(T)$ is an AR-triangle in $\mathcal{C}$. Assume now that $\mathcal{C}^{\mathrm{b}}$ has left AR-triangles and let $W$ be a compact object with local endomorphism ring. Let $(T)$ be as above an ARtriangle in $\mathcal{C}^{\mathrm{b}}$. By hypothesis $(T)$ is an AR-triangle in $\mathcal{C}$. Then by Lemma 6.4 it follows that $W$ is pure-injective.

LEMMA 9.3. Let $\mathcal{T}$ be a full triangulated subcategory of $\mathcal{C}$ which contains the compact objects and consists of pure-injective objects. If $(T): \Sigma^{-1} W \stackrel{\alpha}{\rightarrow} Z \stackrel{\beta}{\rightarrow}$ $X \stackrel{\gamma}{\rightarrow} W$ is a triangle in $\mathcal{C}$ with $W \in \mathcal{T}$, then the following are equivalent:

(i) $(T)$ is an AR-triangle in $\mathcal{C}$.

(ii) $(T)$ is an AR-triangle in $\mathcal{T}$.

If (ii) holds, then $X$ is compact and $W \cong \mathbb{D}_{\mathfrak{m}}(X)$.

Proof. (i) $\Rightarrow$ (ii) Since $W$ is pure-injective, by Lemma 6.4 it follows that $X$ is compact. Therefore $(T)$ lies in $\mathcal{T}$ and then it is an AR-triangle in $\mathcal{T}$. Conversely assume that $(T)$ is an AR-triangle in $\mathcal{T}$. If $X$ is not compact, then the morphism $\gamma$ is pure. Since $W$ is pure-injective it follows that $\gamma=0$ and this is impossible. Hence $X$ is compact. Let $\left(T^{\prime}\right): \Sigma^{-1} \mathbb{D}_{\mathfrak{m}}(X) \rightarrow$ $A \rightarrow X \stackrel{h}{\rightarrow} \mathbb{D}_{\mathfrak{m}}(X)$ be the induced AR-triangle in $\mathcal{C}$. Then we have a morphism of triangles 


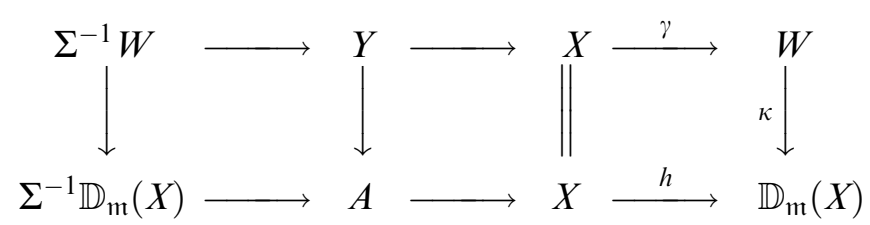

We claim that $\kappa$ is a pure monomorphism. Indeed let $\phi: T \rightarrow W$ be a morphism in $\mathcal{C}$ where $T$ is compact such that $\phi \circ \kappa=0$. If $\phi$ is not zero, then the cofiber $\psi: W \rightarrow S$ of $\phi$ factors through $\Sigma(\alpha)$, since $S$ lies in $\mathcal{T}$. In particular $\gamma \circ \psi=0$. Hence $\psi=\Sigma(\alpha) \circ \tau$ where $\tau: \Sigma(Y) \rightarrow S$. Since $\phi \circ \kappa=0$, there exists $\xi: \mathbb{D}_{\mathfrak{m}}(X) \rightarrow S$ such that $\psi \circ \xi=\kappa$. Then $0=\gamma \circ \psi \circ \xi=\gamma \circ \kappa=h$ and this is impossible. We infer that $\kappa$ is a pure monomorphism and therefore an isomorphism since $W$ is indecomposable pure-injective. This implies that the triangles $(T)$ and $\left(T^{\prime}\right)$ are isomorphic and consequently $(T)$ is an AR-triangle in $\mathcal{C}$.

COROLLARY 9.4. Let $\mathcal{T}$ be a full triangulated Krull-Schmidt subcategory of $\mathcal{C}$ which contains the compact objects and consists of pure-injective objects. If $\mathcal{T}$ has right AR-triangles, then $\mathcal{T}=\mathcal{C}^{\mathrm{b}}$. The converse holds provided that the dual object of any compact object lies in $\mathcal{T}$.

Proof. By Lemma 9.3 it follows that any indecomposable object in $\mathcal{T}$ is compact and therefore the assertion follows from the fact $\mathcal{T}$ is KrullSchmidt.

The above results suggest to look for conditions ensuring that a given (compact) object is pure-injective. In this direction we have the following result which will be useful later in connection with the finite type property.

PROPOSITION 9.5. If $E$ is an object in $\mathcal{C}$, then the following are equivalent.

(i) $E$ is pure-injective.

(ii) $(*) \lim ^{(1)} \mathcal{C}\left(Y_{i}, E\right)=0$, for any filtered system $\left\{Y_{i} \mid i \in I\right\}$ in $\mathcal{C}^{\mathrm{b}}$.

Proof. Since $E$ is pure-injective in $\mathcal{C}$ iff $\mathrm{H}(E)$ is injective in Mod- $\mathcal{C}^{\text {b }}$, it suffices to show that $\mathrm{H}(E)$ is injective iff the vanishing condition $(*)$ holds. Since $\mathrm{H}(E)$ is flat = FP-injective functor, and since in a locally coherent category an object is injective iff it is FP-injective and pure-injective, we have that $\mathrm{H}(E)$ is injective iff $\mathrm{H}(E)$ is pure-injective. By a result of Jensen and Simson, see [43, Corollary 1.3], it follows that $\mathrm{H}(E)$ is pure-injective iff for any flat functor $F$ we have: $\operatorname{Ext}^{n}(F, \mathrm{H}(E))=0, \forall n \geqslant 1$. Since the full subcategory of flat functors contains the projectives and is closed under kernels of epimorphisms, hence under syzygies, we have that $\mathrm{H}(E)$ is pure-injective iff $\operatorname{Ext}^{1}(F, \mathrm{H}(E))=0$, for any flat functor $F$. So let $F \in \operatorname{Mod}^{-} \mathcal{C}^{\mathrm{b}}$ be a flat functor and write $F=\lim \mathrm{H}\left(Y_{i}\right)$ as a filtered colimit of finitely generated projective 
functors, i.e. $\left\{Y_{i} \mid i \in I\right\}$ is a filtered system of compact objects. Then we have the following Roos spectral sequence $\mathrm{E}_{2}^{p, q}=\lim ^{(p)} \operatorname{Ext}^{q}\left(\mathrm{H}\left(Y_{i}\right), \mathrm{H}(E)\right) \Longrightarrow$ $\operatorname{Ext}^{n}(F, \mathrm{H}(E))$ which of course collapses giving isomorphisms, $\forall n \geq 0$ : $\lim ^{(n)} \mathcal{C}\left(Y_{i}, E\right) \cong \operatorname{Ext}^{n}(F, \mathrm{H}(E))$. It follows that $\mathrm{H}(E)$ is pure-injective iff $\overleftarrow{\lim }^{(1)} \mathcal{C}\left(Y_{i}, E\right)=0$.

We recall from [59] that an object $E$ in $\mathcal{C}$ is called endofinite provided that the right $\operatorname{End}_{\mathcal{C}}(E)$-module $\mathcal{C}(X, E)$ has finite length for any compact object $X$ in $\mathcal{C}$. Since $\lim ^{(1)}$ vanishes for filtered systems of finite length modules, Proposition $\overleftarrow{9.5}$ admits the following well known consequence.

COROLLARY 9.6.[50]. Let $E$ be an endofinite object in $\mathcal{C}$. Then $E$ is pureinjective.

COROLLARY 9.7. Assume that $\mathcal{C}$ is $R$-linear over a commutative Noetherian ring $R$. Let $\mathcal{T}$ be a full subcategory of $\mathcal{C}$ containing $\mathcal{C}^{\mathrm{b}}$. Then $\mathcal{T}$ consists of pureinjective objects provided that one of the following conditions holds:

(i) $R$ is a finite product of complete local rings and for all objects $X, Y$ in $\mathcal{T}$, the $R$-module $\mathcal{C}(X, Y)$ is finitely generated.

(ii) For all objects $X, Y$ in $\mathcal{T}$, the $R$-module $\mathcal{C}(X, Y)$ is of finite length.

Moreover in case (ii), any object of $\mathcal{T}$ is endofinite.

Proof. By results of Gruson and Jensen [34] we have that in both cases $\lim ^{(n)} \mathcal{C}\left(Y_{i}, X\right)=0, \forall n \geqslant 1$, for any filtered system $\left\{Y_{i} \mid i \in I\right\}$ of compact

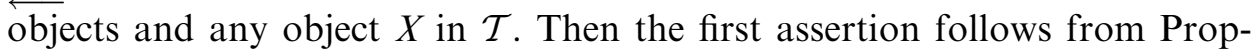
osition 9.5. Now if (ii) holds, it follows that for any compact object $X$ and any object $E$ in $\mathcal{T}$, the $R$-module $\mathcal{C}(X, E)$ has finite length. Since $\operatorname{End}_{\mathcal{C}}(E)$ is a Noetherian $R$-algebra, this implies that $\mathcal{C}(X, E)$ has finite length as an $\operatorname{End}_{\mathcal{C}}(E)$-module. Hence $E$ is endofinite.

The above result suggests naturally the following definition. First recall from Section 4 that a skeletally small $R$-linear triangulated category $\mathcal{T}$ over a commutative ring $R$ is called $R$-finite if one of the following conditions hold:

(I) $R$ is Noetherian and the $R$-module $\mathcal{T}(A, B)$ is of finite length, $\forall A, B \in \mathcal{T}$.

(II) $R$ is Artinian and the $R$-module $\mathcal{T}(A, B)$ is finitely generated, $\forall A, B \in \mathcal{T}$.

(III) $R$ is Noetherian complete and local and the $R$-module $\mathcal{T}(A, B)$ is finitely generated, $\forall A, B \in \mathcal{T}$.

DEFINITION 9.8. Let $\mathcal{C}$ be a compactly generated triangulated $R$-linear category over a commutative ring $R$. We say that $\mathcal{C}$ is compactly $R$-finite if the full subcategory $\mathcal{C}^{\mathrm{b}}$ of compact objects is $R$-finite. 
COROLLARY 9.9. Let $\mathcal{C}$ be a compactly generated compactly $R$-finite triangulated category over a commutative ring $R$. Then we have the following.

(i) $\mathcal{C}^{\mathrm{b}}$ is a Krull-Schmidt category, any compact object is endofinite (hence pure-injective), and any $A R$-triangle in $\mathcal{C}^{b}$ is an AR-triangle in $\mathcal{C}$.

(ii) If $\mathcal{T}$ is a skeletally small full triangulated subcategory of $\mathcal{C}$ which is $R$-finite and contains the compact objects, then the conclusions of (i) hold for $\mathcal{T}$. In addition $\mathcal{T}=\mathcal{C}^{\mathrm{b}}$ provided that $\mathcal{T}$ has right AR-triangles.

Remark 9.10. Let $\mathcal{T}$ be as in Corollary 9.9. For instance we can choose $\mathcal{T}$ to be the thick subcategory of $\mathcal{C}$ generated by the compact objects and the maximal points of $\mathcal{C}$. Following Orlov [67] we define the triangulated category of singularities of $\mathcal{T}$ to be the Verdier quotient $\mathcal{T} / \mathcal{C}^{\mathrm{b}}$ and then $\mathcal{T}$ is called nonsingular if $\mathcal{T} / \mathcal{C}^{\mathrm{b}}=0$. Corollary 9.9 shows that $\mathcal{T}$ is non-singular if $\mathcal{T}$ has right AR-triangles. The converse holds if $\mathcal{T}$ contains all maximal points. This happens for instance for the bounded derived category $\mathbf{D}^{\mathrm{b}}(\operatorname{coh} \mathbb{X})$ of coherent sheaves over a Noetherian scheme $\mathbb{X}$ which is of finite type over a field. Then the above results show that $\mathbb{X}$ is non-singular iff $\mathbf{D}^{\mathrm{b}}(\operatorname{coh} \mathbb{X})$ has AR-triangles.

EXAMPLE 9.11. Let $\Lambda$ be a Noetherian $R$-algebra over a commutative Noetherian ring and assume that $\Lambda$ is Artinian or $R$ is complete and local. By Subsection 4.2, the unbounded derived category $\mathbf{D}$ (Mod- $\Lambda$ ) is compactly $R$-finite. Moreover it is not difficult to see that the bounded derived category $\mathbf{D}^{\mathrm{b}}(\bmod -\Lambda)$ is Krull-Schmidt and for any two complexes $X$ and $Y$ in $\mathbf{D}^{\mathrm{b}}(\bmod -\Lambda)$, the $R$-module $\mathbf{D}^{\mathrm{b}}(\bmod -\Lambda)[X, Y]$ is finitely generated. In particular $\mathbf{D}^{\mathrm{b}}(\bmod -\Lambda)$ consists of pure-injective objects in $\mathbf{D}(\operatorname{Mod}-\Lambda)$. If $R$ is Artinian, then any object of $\mathbf{D}^{\mathrm{b}}(\bmod -\Lambda)$ is endofinite.

Since $\mathbf{D}^{\mathrm{b}}(\bmod -\Lambda)$ contains the compact objects and consists of pure-injectives, we define the triangulated category of singularities $\mathbf{D}_{\text {sing }}($ Mod- $\Lambda$ ) of $\Lambda$ to be the Verdier quotient $\mathbf{D}^{\mathrm{b}}(\bmod -\Lambda) / \mathcal{H}^{\mathrm{b}}\left(\mathcal{P}_{\Lambda}\right)$. Note that $\left.\mathbf{D}_{\text {sing }}(\operatorname{Mod}-\Lambda)\right)$ is triangle equivalent to the Spanier-Whitehead category of the stable category mod- $\Lambda$ modulo projectives, see [46] or [17, Corollary 3.9]. In Theorem 9.16 we shall show that $\Lambda$ is non-singular, i.e. $\mathbf{D}_{\text {sing }}(\operatorname{Mod}-\Lambda)=0$, iff $\Lambda$ is Artinian of finite global dimension.

EXAMPLE 9.12. Let $R$ be a finite dimensional differential graded algebra (DGA) over a field $k$ such that $R^{i}=0$ for $i<0, R^{0}=k$ and $R^{1}=0$. Then, by a result of Jørgensen [44, Lemma 3.6], the full subcategory $\mathbf{D}^{\mathrm{f}}(R)$ of the unbounded derived category $\mathbf{D}(R)$ consisting of complexes with finite dimensional cohomology, has finite-dimensional Hom-spaces. It follows that $\mathbf{D}(R)$ is compactly $k$-finite and any complex in $\mathbf{D}^{\mathbf{f}}(R)$ is endofinite. For instance one can take $R$ to be the singular cochain DGA $\mathcal{C}^{*}(X ; k)$ of a simply connected topological space $X$ over $k$ with $\operatorname{dim}_{k} \mathrm{H}^{*}(X ; k)<\infty$. We refer to 
[44] for an analysis of Auslander-Reiten theory in this context. In particular in [44, Theorem 6.3] it is proved that $\mathbf{D}\left(C^{*}(X ; k)\right)^{\mathrm{b}}$ has AR-triangles iff $X$ admits Poincaré duality over $k$, hence, iff $\mathbf{D}\left(C^{*}(X ; k)\right)^{\mathrm{b}}$ admits a Serre functor. It follows that in the Ziegler spectrum of $\mathbf{D}\left(C^{*}(X ; k)\right)$, the finite points ( $=$ indecomposable objects in the thick subcategory of $\mathbf{D}\left(C^{*}(X ; k)\right)$ generated by $\left.\mathcal{C}^{*}(X ; k)\right)$ coincide with the maximal points, and any AR-triangle in $\mathbf{D}\left(C^{*}(X ; k)\right)^{\mathrm{b}}$ remains an AR-triangle in $\mathbf{D}\left(C^{*}(X ; k)\right)$.

\subsection{AUSLANDER-REITEN TRIANGLES AND GORENSTEIN RINGS}

In this subsection we study existence of AR-triangles in the unbounded derived category $\mathbf{D}(\operatorname{Mod}-\Lambda)$, where $\Lambda$ is a Noetherian $R$-algebra over a commutative Noetherian ring $R$. We assume throughout that $R$ is Artinian or complete and local. If $I$ is the minimal injective cogenerator of Mod- $R$, then, as in Section 4, let $\mathrm{D}$ be any one of the duality functors $\operatorname{Hom}_{R}(-, I):(\operatorname{Mod}-R)^{\mathrm{op}} \rightarrow($ Mod- $R$ ), or $\mathbb{R} \operatorname{Hom}_{R}(-, I): \mathbf{D}(\operatorname{Mod}-R)^{\text {op }} \rightarrow \mathbf{D}(\operatorname{Mod}-R), \quad$ or $\quad \mathbb{R} \operatorname{Hom}_{R}(-, I)=$ $\mathbb{R} \operatorname{Hom}_{\Lambda}(-, \mathrm{D}(\Lambda)): \mathbf{D}(\operatorname{Mod}-\Lambda)^{\mathrm{op}} \rightarrow \mathbf{D}\left(\operatorname{Mod}-\Lambda^{\mathrm{op}}\right)$.

We begin with the following preliminary result whose proof is identical with the proof of Theorems 9.15 and 9.16 below and therefore we omit it.

PROPOSITION 9.13. Let $R$ be a commutative complete local Noetherian ring.

(i) $\mathcal{H}^{\mathrm{b}}\left(\mathcal{P}_{R}\right)$ has AR-triangles iff $\mathrm{id} R<\infty$ and the Krull dimension of $R$ is zero, equivalently $R$ is an Artinian Gorenstein ring.

(ii) $\mathbf{D}^{\mathrm{b}}$ (mod- $R$ ) has AR-triangles iff $R$ is regular Artinian.

We shall now see that the conclusions of Proposition 9.13 continue to hold for Noetherian $R$-algebras. We begin with the following result. Notice that the equivalence (i) $\Leftrightarrow$ (ii) below extends slightly a result of Happel [38, Theorem 3.4].

PROPOSITION 9.14. The following are equivalent:

(i) $\Lambda$ is Artinian and $\operatorname{id}_{\Lambda} \Lambda<\infty$.

(ii) $\mathcal{H}^{\mathrm{b}}\left(\mathcal{P}_{\Lambda}\right)$, resp. $\mathcal{H}^{\mathrm{b}}\left(\mathcal{P}_{\Lambda^{\mathrm{p}}}\right)$, has right, resp. left, AR-triangles.

(iii) $\mathcal{H}^{\mathrm{b}}\left(\mathcal{P}_{\Lambda}\right)$, resp. $\mathcal{H}^{\mathrm{b}}\left(\mathcal{P}_{\Lambda^{\mathrm{op}}}\right)$, admits a right, resp. left, Serre functor.

(iv) $\operatorname{Max}(\mathbf{D}(\operatorname{Mod}-\Lambda)) \subseteq \operatorname{Fin}(\mathbf{D}(\operatorname{Mod}-\Lambda))$.

(v) $\operatorname{Fin}\left(\mathbf{D}\left(\operatorname{Mod}-\Lambda^{\mathrm{op}}\right)\right) \subseteq \operatorname{Max}\left(\mathbf{D}\left(\operatorname{Mod}-\Lambda^{\mathrm{op}}\right)\right)$.

Proof. Since $\mathcal{H}^{\mathrm{b}}\left(\mathcal{P}_{\Lambda}\right)$ is $R$-finite, our previous results and the duality $\mathcal{H}^{\mathrm{b}}\left(\mathcal{P}_{\Lambda}\right) \rightarrow \mathcal{H}^{\mathrm{b}}\left(\mathcal{P}_{\Lambda^{\text {op }}}\right)$ show that the last four statements are equivalent. If (i) holds, then $\mathrm{D}(\Lambda)_{\Lambda}$ is compact in $\mathbf{D}(\operatorname{Mod}-\Lambda)$, hence $\mathbb{R H o m}_{\Lambda}(\mathrm{D}(\Lambda),-)$ preserves coproducts. By [64, Theorem 5.1] this implies that $-\otimes_{\Lambda}^{\mathbb{L}} \mathrm{D}(\Lambda)$ preserves compact objects. Since $\mathbb{D}_{\mathfrak{m}}(X) \cong X \otimes_{\Lambda}^{\mathbb{L}} \mathrm{D}(\Lambda)$ for any perfect complex 
$X$, it follows that $\mathcal{H}^{\mathrm{b}}\left(\mathcal{P}_{\Lambda}\right)$ has right AR-triangles. Conversely if (ii) holds, then let $\mathrm{S}$ be the right Serre functor of $\mathcal{H}^{\mathrm{b}}\left(\mathcal{P}_{\Lambda}\right)$. Then the isomorphisms

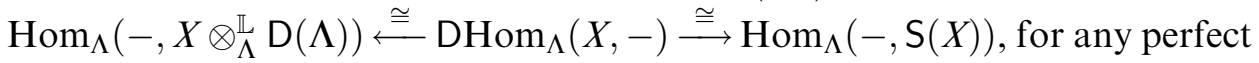
complex $X$, show that $\mathrm{D}(\Lambda)$ is quasi-isomorphic to $\mathrm{S}(\Lambda)$ and $\mathrm{S} \cong$ $-\left.\otimes \mathbb{L}_{\Lambda}^{\mathbb{L}} \mathrm{D}(\Lambda)\right|_{\mathcal{H}^{\mathrm{b}}\left(\mathcal{P}_{\Lambda}\right)}$. Since $\mathrm{S}(\Lambda)$ lies in $\mathcal{H}^{\mathrm{b}}\left(\mathcal{P}_{\Lambda}\right)$ it follows that $\mathrm{D}(\Lambda)$ lies in mod$\Lambda$ and $\operatorname{pdD}(\Lambda)_{\Lambda}<\infty$. In other words, $\Lambda$ is Artinian and $\operatorname{id}_{\Lambda} \Lambda<\infty$.

The following consequence generalizes results of Happel [38], see also [57].

THEOREM 9.15. Let $\Lambda$ be an Noetherian R-algebra over a commutative Noetherian ring $R$, where $R$ is complete local or Artinian. Then the following are equivalent.

(i) $\Lambda$ is an Artinian Gorenstein ring.

(ii) $\mathcal{H}^{\mathrm{b}}\left(\mathcal{P}_{\Lambda}\right)$ has AR-triangles, resp. $\mathcal{H}^{\mathrm{b}}\left(\mathcal{P}_{\Lambda}\right)$ is dualizing, resp. $\mathcal{H}^{\mathrm{b}}\left(\mathcal{P}_{\Lambda}\right)$ admits a Serre functor.

(iii) $-\otimes_{\Lambda}^{\mathbb{L}} \mathrm{D}(\Lambda)$ is a Serre functor in $\mathcal{H}^{\mathrm{b}}\left(\mathcal{P}_{\Lambda}\right)$.

(iv) $-\otimes \mathbb{L}_{\Lambda} \mathrm{D}(\Lambda): \mathbf{D}($ Mod- $\Lambda) \rightarrow \mathbf{D}$ (Mod- $\left.\Lambda\right)$ is a triangle equivalence.

(v) $\operatorname{Max}(\mathbf{D}(\operatorname{Mod}-\Lambda))=\operatorname{Fin}(\mathbf{D}(\operatorname{Mod}-\Lambda))$.

Proof. Follows directly from Proposition 9.14 and the fact that a coproduct preserving exact functor $F$ between compactly generated triangulated categories is an equivalence iff $F$ restricts to an equivalence between the full subcategories of compact objects, see for instance [47, Lemma 4.2].

Let $\mathbf{D}_{\text {noeth }}^{\mathrm{b}}(\operatorname{Mod}-\Lambda)=\mathbf{D}^{\mathrm{b}}(\bmod -\Lambda)$, resp. $\mathbf{D}_{\text {art }}^{\mathrm{b}}(\operatorname{Mod}-\Lambda)$, be the full subcategory of $\mathbf{D}^{\mathrm{b}}(\operatorname{Mod}-\Lambda)$ consisting of all complexes with Noetherian, resp. Artinian, cohomology. The equivalence (i) $\Leftrightarrow$ (ii) below generalizes slightly another basic result of Happel [37, Corollary 1.4] proved by different methods.

THEOREM 9.16. Let $\Lambda$ be a Noetherian $R$-algebra over a commutative Noetherian complete local ring. Then the following are equivalent.

(i) $\Lambda$ is Artinian and gl.dim $\Lambda<\infty$.

(ii) $\mathbf{D}_{\text {noeth }}^{\mathrm{b}}($ Mod- $\Lambda)$ has right AR-triangles.

(iii) $\mathbf{D}_{\text {art }}^{\mathrm{b}}(\operatorname{Mod}-\Lambda)$ has left AR-triangles.

(iv) $\mathbf{D}_{\text {noeth }}^{\mathrm{b}}(\operatorname{Mod}-\Lambda)$, resp. $\mathbf{D}_{\text {art }}^{\mathrm{b}}(\operatorname{Mod}-\Lambda)$, is a dualizing $R$-variety.

(v) $\mathbf{D}_{\text {noeth }}^{b}(\operatorname{Mod}-\Lambda)$, resp. $\mathbf{D}_{\text {art }}^{b}(\operatorname{Mod}-\Lambda)$, admits a Serre functor.

(vi) $\operatorname{Ind}\left(\mathbf{D}^{\mathrm{b}}(\bmod -\Lambda)\right)=\operatorname{Max}(\mathbf{D}(\operatorname{Mod}-\Lambda))$.

(vii) $\Lambda$ is Artinian and non-singular, i.e. $\mathbf{D}_{\text {sing }}(\operatorname{Mod}-\Lambda)=0$.

If $(\mathrm{i})$ holds, then $\mathbf{D}_{\text {noeth }}^{\mathrm{b}}(\operatorname{Mod}-\Lambda)=\mathbf{D}_{\text {art }}^{\mathrm{b}}(\operatorname{Mod}-\Lambda)=\mathbf{D}^{\mathrm{b}}(\bmod -\Lambda)=\mathcal{H}^{\mathrm{b}}\left(\mathcal{P}_{\Lambda}\right)$ has AR-triangles and admits a Serre functor which is given by $-\otimes_{\Lambda}^{\mathbb{L}} \mathrm{D}(\Lambda)$. 
Proof. (i) $\Rightarrow$ (ii), (iii) If (i) holds, then $\bmod -\Lambda=\operatorname{noeth}(\Lambda)=\operatorname{art}(\Lambda)$ and $\Lambda$ is Gorenstein. Then $\mathcal{H}^{\mathrm{b}}\left(\mathcal{P}_{\Lambda}\right)=\mathbf{D}_{\text {noeth }}^{\mathrm{b}}(\operatorname{Mod}-\Lambda)=\mathbf{D}_{\text {art }}^{\mathrm{b}}(\operatorname{Mod}-\Lambda)$ has AR-triangles by Theorem 9.15. If (ii) holds, then since $\mathbf{D}_{\text {noeth }}^{\mathrm{b}}(\operatorname{Mod}-\Lambda)$ is $R$-finite and contains the compact objects, by Corollary 9.4 and Proposition 9.14, we have that $\Lambda$ is Artinian and $\mathcal{H}^{\mathrm{b}}\left(\mathcal{P}_{\Lambda}\right)=\mathbf{D}_{\text {noeth }}^{\mathrm{b}}(\operatorname{Mod}-\Lambda)$. Hence gl.dim $\Lambda<\infty$. The remaining assertions follow from our previous results and the fact that the functor $\mathrm{D}: \mathbf{D}(\operatorname{Mod}-\Lambda) \rightarrow \mathbf{D}\left(\operatorname{Mod}-\Lambda^{\mathrm{op}}\right)$ induces a duality $\mathbf{D}_{\text {art }}^{\mathrm{b}}($ Mod$\Lambda) \rightarrow \mathbf{D}_{\text {noeth }}^{\mathrm{b}}\left(\operatorname{Mod}-\Lambda^{\mathrm{op}}\right)$ and for any indecomposable perfect complex $X$ we have $\mathbb{D}_{\mathfrak{m}}(X) \cong X \otimes_{\Lambda}^{\mathbb{L}} \mathrm{D}(\Lambda) \in \mathbf{D}_{\text {art }}^{\mathrm{b}}(\operatorname{Mod}-\Lambda)$.

EXAMPLE 9.17. Let $R$ be a DGA as in Example 9.12. Then $\mathbf{D}^{f}(R)$ has (right) AR-triangles iff $R$ is non-singular, i.e. $\mathbf{D}^{\mathrm{f}}(R)=\mathbf{D}(R)^{\mathrm{b}}$, that is, any DG $R$-module $M$ with $\operatorname{dimH}^{*}(M)<\infty$ is finitely built from $R$. This follows from Corollary 9.4 since the dual object of any compact object lies in the image of $-\otimes_{R}^{\mathbb{L}} \mathrm{D}(R)$.

EXAMPLE 9.18. Let $0 \rightarrow \mathcal{A} \rightarrow \mathcal{B} \rightarrow \mathcal{C} \rightarrow 0$ be an exact sequence of triangulated categories. Then it is not true that $\mathcal{B}$ has AR-triangles if $\mathcal{A}$ and $\mathcal{C}$ do so. Indeed let $\Lambda$ be a Gorenstein $R$-algebra of infinite global dimension. Then we have a short exact sequence of $R$-finite triangulated categories $0 \rightarrow \mathcal{H}^{\mathrm{b}}\left(\mathcal{P}_{\Lambda}\right) \rightarrow \mathbf{D}^{\mathrm{b}}(\bmod -\Lambda) \rightarrow \underline{\mathrm{CM}}(\Lambda) \rightarrow 0$, where $\underline{\mathrm{CM}}(\Lambda)$ is the stable category of Cohen-Macaulay modules which coincides with $\mathbf{D}_{\text {sing }}(\operatorname{Mod}-\Lambda)$, see [17, Corollary 6.14]. Clearly both $\mathcal{H}^{\mathrm{b}}\left(\mathcal{P}_{\Lambda}\right)$ and $\mathrm{CM}(\Lambda)$ have AR-triangles, but this is not true for $\mathbf{D}^{\mathrm{b}}(\bmod -\Lambda)$ since $\operatorname{gl} \cdot \operatorname{dim} \Lambda=\infty$.

\subsection{CONSTRUCTIONS OF GORENSTEIN ALGEBRAS}

Recall from [9] that an Artin algebra $\Lambda$ is called Cohen-Macaulay provided that there exists a finitely generated $\Lambda$-bimodule ${ }_{\Lambda} \omega_{\Lambda}$, called dualizing bimodule, such that the functor $-\otimes_{\Lambda} \omega$ induces an equivalence between the subcategory of $\Lambda$-modules with finite projective dimension and the subcategory of $\Lambda$-modules with finite injective dimension. It is easy to see that Gorenstein Artin algebras, in particular self-injective Artin algebras and Artin algebras of finite global dimension, are Cohen-Macaulay, see [9] for more examples. For the notion of trivial extension of rings used below we refer to [16].

COROLLARY 9.19. Let $\Lambda$ be a Cohen-Macaulay Artin algebra with dualizing bimodule $\omega$ and let $\Gamma:=\Lambda \ltimes \omega$ be the trivial extension algebra of $\Lambda$ by $\omega$. Then the category $\mathcal{H}^{\mathrm{b}}\left(\mathcal{P}_{\Lambda \ltimes \omega}\right)$ has AR-triangles. Moreover if $X$ is an indecomposable perfect complex in $\mathbf{D}(\operatorname{Mod}-\Lambda))$, then we have the following isomorphisms in $\mathbf{D}(\operatorname{Mod}-\Gamma)$ :

$$
\begin{aligned}
& \tau_{\Gamma}^{+}\left(X \otimes_{\Lambda}^{\mathbb{L}} \Gamma\right) \stackrel{\cong}{\rightarrow} \mathbb{D}_{\mathfrak{n}}\left(X \otimes_{\Lambda}^{\mathbb{L}} \Gamma\right)[-1] \stackrel{\cong}{\rightarrow} \mathbb{R} \operatorname{Hom}_{\Lambda}\left(\Gamma, \mathbb{D}_{\mathfrak{m}}(X)\right)[-1] \\
& \stackrel{\cong}{\rightarrow} \operatorname{Hom}_{\Lambda}\left(\Gamma, \tau_{\Lambda}^{+}(X)\right) \text {. }
\end{aligned}
$$


Proof. By [16, Corollary 4.14], the trivial extension of a Cohen-Macaulay algebra by a dualizing bimodule is Gorenstein. So the assertions follow from Theorem 9.15 and the fact that, by Corollary $4.5, \tau^{+}=-\otimes_{\Lambda}^{\mathbb{L}} \mathrm{D}(\Lambda)[-1]$ and similarly for $\Gamma$.

If $R$ is a commutative Noetherian Cohen-Macaulay ring with dualizing module $\omega$, then working as in Corollary 9.19 and using Proposition 9.13 we have that $\mathcal{H}^{\mathrm{b}}\left(\mathcal{P}_{R \ltimes \omega}\right)$ has AR-triangles iff $R$ is Artinian.

Now let $\mathcal{C}$ be a compactly generated triangulated category which admits a compact generator $T$ with endomorphism ring $\Lambda_{T}$. If $J$ is an injective cogenerator of $\operatorname{Mod}-\Lambda_{T}$, then the $J$-dual object $\mathbb{D}^{J}(T)$ is a pure-injective cogenerator of $\mathcal{C}$. A compact object $P$ in $\mathcal{C}$ is called a Wakamatsu compact object if the pure-injective cogenerator $\mathbb{D}^{J}(T)$ of $\mathcal{C}$ lies in the localizing subcategory of $\mathcal{C}$ generated by $P$. Finally we say that a DG-algebra $\Gamma$ is Gorenstein if $\mathbf{D}(\Gamma)^{\mathrm{b}}$ has AR-triangles.

THEOREM 9.20. Let $\Lambda$ be a Noetherian R-algebra and assume that either $R$ is Artinian or $R$ is Noetherian, complete and local. Then the following are equivalent.

(i) $\Lambda$ is Artinian Gorenstein.

(ii) For any Wakamatsu perfect complex $P$, the DG-algebra $\Gamma$ of endomorphisms of $P$ is Gorenstein with Artinian cohomology.

(iii) For any perfect complex $P$, the DG-algebra $\Gamma$ of endomorphisms of $P$ is Gorenstein with Artinian cohomology, provided that $P$ is compact when considered as an object in $\mathbf{D}(\Gamma)$.

Proof. Clearly (ii), (iii) $\Rightarrow$ (i) since the compact generator $\Lambda$ is Wakamatsu and satisfies the condition in (iii). Assume now that $\Lambda$ is Artinian Gorenstein. Then, by Theorem 9.15, the subcategory $\mathcal{H}^{\mathrm{b}}\left(\mathcal{P}_{\Lambda}\right)$ has AR-triangles and the pure-injective cogenerator $D(\Lambda)$ of $\mathbf{D}(\operatorname{Mod}-\Lambda)$ is a compact generator. We now fix a perfect complex $P$ with DG endomorphism algebra $\Gamma$ and let $\mathcal{X}$ be the localizing subcategory of $\mathbf{D}(\operatorname{Mod}-\Lambda)$ generated by $P$. By $[22$, Theorem IV.2.1], $\mathcal{X}$ is the torsion class of a torsion triple $(\mathcal{X}, \mathcal{Y}, \mathcal{Z})$ in $\mathbf{D}(\operatorname{Mod}-\Lambda)$ and $\mathcal{X}^{\mathrm{b}}$ coincides with the thick subcategory of $\mathbf{D}(\operatorname{Mod}-\Lambda)$ generated by $P$. Moreover the functor $-\otimes_{\Gamma}^{\mathbb{L}} P_{\Lambda}: \mathbf{D}(\Gamma) \rightarrow \mathbf{D}(\operatorname{Mod}-\Lambda)$ is fully faithful and induces a triangle equivalence $-\otimes \frac{\mathbb{L}}{\Gamma} P_{\Lambda}: \mathbf{D}(\Gamma) \stackrel{\approx}{\underset{\mathcal{X}}{\longrightarrow}}$ which restricts to a triangle equivalence $-\otimes_{\Gamma}^{\mathbb{L}} P_{\Lambda}: \mathbf{D}(\Gamma)^{\mathrm{b}} \stackrel{\approx}{\underset{ }{\longrightarrow}} \mathcal{X}^{\mathrm{b}}$. If the complex $P$ is Wakamatsu, then $\mathrm{D}(\Lambda)$ lies in $\mathcal{X}$. Since $\mathcal{X}$ is localizing and contains the compact generator $\mathrm{D}(\Lambda)$, we infer that $\mathcal{X}=\mathbf{D}(\operatorname{Mod}-\Lambda)$. Therefore $\mathbf{D}(\Gamma)^{\mathrm{b}} \stackrel{\approx}{\longrightarrow} \mathcal{X}^{\mathrm{b}}$ has AR-triangles or equivalently it is dualizing. This implies that the cohomology of $\Gamma$ is Artinian. If the complex $P$ is perfect in $\mathbf{D}(\Gamma)$, then by a result of Keller, see [22, Proposition IV.3.5], the functor $-\otimes_{\Gamma}^{\mathbb{L}} P_{\Lambda}$ preserves products. As in [22, Theorem 3.4], this implies that $\mathcal{X}$ is closed under products in 
$\mathbf{D}($ Mod- $\Lambda)$. By Proposition 5.14 there exists a torsion quadruple $(\mathcal{W}, \mathcal{X}, \mathcal{Y}, \mathcal{Z})$ in $\mathbf{D}(\operatorname{Mod}-\Lambda)$ which restricts to a torsion triple $\left(\mathcal{W}^{\mathrm{b}}, \mathcal{X}^{\mathrm{b}}, \mathcal{Y}^{\mathrm{b}}\right)$ in $\mathcal{H}^{\mathrm{b}}\left(\mathcal{P}_{\Lambda}\right)$. Then Lemma 5.9 implies that $\mathcal{X}^{\mathrm{b}}$ has AR-triangles and as above we infer that $\Gamma$ is Gorenstein with Artinian cohomology.

Let $\Lambda$ be an Artin algebra and $T$ a finitely generated $\Lambda$-module. We call $T$ a Wakamatsu module, if there exists an exact sequence $\cdots \rightarrow T_{1} \rightarrow T_{0} \rightarrow \mathrm{D}(\Lambda) \rightarrow 0$ in $\bmod -\Lambda$, where each $T_{i}$ lies in $\operatorname{add}(T)$. Examples of Wakamatsu modules include the so-called Wakamatsu (co)tilting modules, see [22], [78]. Recall that the Yoneda Ext-algebra of a finitely generated $\Lambda$-module $T$ is defined by $E(T)=\oplus_{n \geqslant 0} \operatorname{Ext}_{\Lambda}^{n}(T, T)$.

COROLLARY 9.21. The algebra $\Lambda$ is Gorenstein iff for any Wakamatsu $\Lambda$ module $T$ of finite projective dimension, the Yoneda Ext-algebra $E(T)$ of $T$ is Gorenstein.

Proof. It follows easily that $T$ is a Wakamatsu perfect complex, see the proof of Theorem IV.3.4 of [22]. Then the assertion follows from Theorem 9.20 .

COROLLARY 9.22. Let $\Lambda$ be a Gorenstein Artin algebra and $T$ a finitely generated Ext-orthogonal $\Lambda$-module with endomorphism ring $\Gamma$ such that $\operatorname{pd} T_{\Lambda}<\infty$. If $T$ is Wakamatsu or $\operatorname{pd}_{\Gamma} T<\infty$, then $\Gamma$ is Gorenstein. In particular if $\operatorname{gl} \cdot \operatorname{dim} \Lambda<\infty$, then $\operatorname{End}_{\Lambda}(T)$ is Gorenstein for any Wakamatsu Extorthogonal $\Lambda$-module $T$.

We close this section with a construction of Gorenstein (DG-)algebras and triangulated categories with AR-triangles starting from finitely generated Cohen-Macaulay modules over an Artin algebra $\Lambda$. Recall from [9] that a finitely generated $\Lambda$-module $X$ is called Cohen-Macaulay if $\operatorname{Ext}_{\Lambda}^{n}(X, \Lambda)=0$, $\forall n \geq 1$, and there exists an exact sequence $0 \rightarrow X \rightarrow P^{0} \rightarrow \cdots \rightarrow$ $P^{n} \stackrel{f^{n}}{\rightarrow} P^{n+1} \rightarrow \cdots$, where the $P^{n}$ are projective and $\operatorname{Ext}_{\Lambda}^{t}\left(\operatorname{Ker}\left(f^{n}\right), \Lambda\right)=0$, $\forall t \geq 1, \forall n \geqslant 0$. Let $\mathcal{U}$ be a subcategory of $\bmod -\Lambda$. We denote by $\lim \mathcal{U}$ the full subcategory of Mod- $\Lambda$ formed by the filtered colimits of modules from $\mathcal{U}$. We recall from [20] that a subcategory $\mathcal{U}$ of mod- $\Lambda$ is called projectively thick if $(\alpha) \mathcal{U}$ is closed under extensions and kernels of epimorphisms, $(\beta) \mathcal{U}$ contains the projectives, and $(\gamma) \mathcal{U}$ is closed under cokernels of maps $g: X \rightarrow Y$ such that $\operatorname{Hom}_{\Lambda}(g, \Lambda)$ is surjective. Notice that, by [20], if $\mathcal{U}$ is a projectively thick subcategory of mod- $\Lambda$ consisting of Cohen-Macaulay modules, then $\mathcal{U}$, resp.

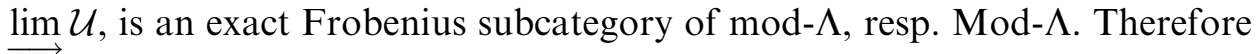
by [36], the stable categories $\underline{\mathcal{U}}$ and $\underset{\lim }{\longrightarrow} \mathcal{U}$ modulo projectives are triangulated. 
THEOREM 9.23. Let $\Lambda$ be an Artin algebra and let $\mathcal{X}$ be a projectively thick subcategory of mod- $\Lambda$ consisting of Cohen-Macaulay modules. If $\mathcal{X}$ is contravariantly or covariantly finite in mod- $\Lambda$, then there exists a Gorenstein DG-algebra $\Gamma$ and a triangle equivalence $\lim \mathcal{X} \underset{\sim}{\rightarrow} \mathbf{D}(\Gamma)$ which induces $a$ triangle equivalence $\underline{\lim } \mathcal{\longrightarrow} \underset{\rightarrow}{\stackrel{\approx}{\rightarrow}} \mathbf{D}(\Gamma)^{\mathrm{b}}$.

Proof. It is proved in [20] that the stable category $\underline{\lim } \mathcal{X}$ is a compactly generated triangulated category and $(\underline{\lim \mathcal{X}})^{\mathrm{b}}=\underline{\mathcal{X}}$. Also, by [20], contravariant finiteness of $\mathcal{X}$ is equivalent to covariant finiteness. Now it is not difficult to see that the right $\mathcal{X}$-approximations $\underline{X}_{S(i)}$ of the simple $\Lambda$-modules $\{S(1), \ldots, S(n)\}$ form a generating set of compact objects for $\stackrel{\lim }{\longrightarrow}$ and therefore by Theorem IV.2.2 of [22], $\lim _{\longrightarrow} \mathcal{X}$ is triangle equivalent to the unbounded derived category $\mathbf{D}(\Gamma)$, where $\Gamma$ is the DG-algebra of endomorphims of the compact generator $\oplus_{i=1}^{n} \underline{X}_{S(i)}$ of $\underline{\lim } \mathcal{X}$. Therefore $\underline{\mathcal{X}}$ is triangle equivalent to $\mathbf{D}(\Gamma)^{\mathrm{b}}$. Since $\mathcal{X}$ is functorially finite in $\bmod -\Lambda$, it follows from [11, Theorem 2.4] that the exact category $\mathcal{X}$ has relative AR-sequences. Clearly this implies that $\mathcal{X}$ has AR-triangles. Hence the DG-algebra $\Gamma$ is Gorenstein.

Note that if $\Lambda$ is Gorenstein, or more generally virtually Gorenstein in the sense of [20], then we can choose $\mathcal{X}$ to be the full subcategory of CohenMacaulay modules.

\section{Categories Of Finite Type, Endofinite Objects and Auslander-Reiten Triangles}

In this section we study compactly generated triangulated categories enjoying finiteness conditions which are of interest in representation theory, for instance pure-semisimplicity or the finite type property, in connection with Auslander-Reiten theory and the structure of the Ziegler spectrum.

Throughout $\mathcal{C}$ denotes a compactly generated triangulated category.

\subsection{CATEGORIES OF FINITE TYPE AND AUSLANDER-REITEN TRIANGLES}

Recall from [18] that $\mathcal{C}$ is called pure-semisimple if any pure triangle in $\mathcal{C}$ splits. Equivalently any object of $\mathcal{C}$ is pure-projective, resp. pure-injective. It is proved in [18, Section 9] that $\mathcal{C}$ is pure-semisimple iff the functor $\mathrm{H}: \mathcal{C} \rightarrow$ Mod- $\mathcal{C}^{\text {b }}$ induces an equivalence $\mathcal{C} \underset{\sim}{\stackrel{\approx}{\rightarrow}} \operatorname{Flat}\left(\mathcal{C}^{\mathrm{b}}\right)$ iff the functor category Mod- $\mathcal{C}^{\text {b }}$ is locally Noetherian, i.e. Mod- $\mathcal{C}^{\mathrm{b}}$ admits a set of Noetherian generators. Then $\mathcal{C}$ is of finite type if the category Mod- $\mathcal{C}^{\mathrm{b}}$ is locally finite, i.e. admits a set of generators of finite length. 
LEMMA 10.1. If $\mathcal{C}$ is pure-semisimple, then $\mathrm{Zg}(\mathcal{C})=\operatorname{lnd}\left(\mathcal{C}^{\mathrm{b}}\right)$ and the category $\mathcal{C}^{\mathrm{b}}$ is Krull-Schmidt with right AR-triangles which are AR-triangles in $\mathcal{C}$.

Proof. By Theorem 9.3 of [18], $\mathcal{C}^{\mathrm{b}}$ is Krull-Schmidt and any indecomposable object is compact. This implies that $\operatorname{Zg}(\mathcal{C})=\operatorname{lnd}\left(\mathcal{C}^{\mathrm{b}}\right)$, hence $\mathcal{C}^{\mathrm{b}}$ has right AR-triangles.

In general if $\mathcal{C}$ is pure-semisimple, then $\mathcal{C}$ is not necessarily of finite type, see [18, Example 12.19] or Example 10.5 below. The following result, which generalizes a result of Zimmermann-Huisgen [82, Theorem $\mathrm{A}^{\prime}$ ] from module theory, shows that the missing item is the existence of left AR-triangles in $\mathcal{C}^{\mathrm{b}}$ :

THEOREM 10.2. The following are equivalent:

(i) $\mathcal{C}$ is of finite type.

(ii) $\mathcal{C}$ is pure-semisimple and $\mathcal{C}^{b}$ has left AR-triangles.

(iii) $\mathcal{C}$ is pure-semisimple and any finite point is maximal.

(iv) $\mathcal{C}$ is pure-semisimple and any finite point is isolated.

Proof. (i) $\Rightarrow$ (ii) By Lemma $10.1, \mathcal{C}$ is pure-semisimple and $\mathcal{C}^{\text {b }}$ is KrullSchmidt. Let $W$ be an indecomposable compact object. Since $\mathcal{C}$ is of finite type, the functor category Mod- $\mathcal{C}^{\mathrm{b}}$ is locally finite and this implies that the finitely generated projective functor $\mathrm{H}(Y)$ has a simple subfunctor $S$, see [2, Theorem 2.10]. Then $S$ admits a projective cover and by Theorem 8.10, $W$ is the source of an AR-triangle in $\mathcal{C}^{b}$.

(ii) $\Rightarrow$ (i) Since $\mathcal{C}$ is pure-semisimple, Mod- $\mathcal{C}^{b}$ is locally Noetherian and, by [2, Theorem 2.10], it suffices to show that any non-zero functor $M \in \operatorname{Mod}-\mathcal{C}^{\mathrm{b}}$ has a simple subfunctor. Since $M \neq 0$, there exists a non-zero morphism $\alpha: \mathrm{H}(X) \rightarrow M$ for some compact $T$, and we have an exact sequence $0 \rightarrow G \rightarrow \mathrm{H}(X) \rightarrow F \rightarrow 0$, where $F=\operatorname{Im}(\alpha)$. Since Mod- $\mathcal{C}^{\mathrm{b}}$ is locally Noetherian, it follows that $\operatorname{Ker}(\alpha)$ is finitely generated and therefore $F$ is finitely presented. Since simple subfunctors of $F$ are simple subfunctors of $M$, it suffices to show that any finitely presented functor $F$ contains a simple subfunctor. Let $\mu: F \hookrightarrow \mathrm{H}(E)$ be the injective envelope of $F$ in Mod- $\mathcal{C}^{\mathrm{b}}$. Then $E$ is pure-injective and therefore $E$ is compact since $\mathcal{C}$ is pure-semisimple. Let $Z$ be an indecomposable direct summand of $E$ which admits a non-zero morphism $f: F \rightarrow \mathrm{H}(Z)$. Then $Z$ is compact and therefore there exists an AR-triangle $Z \rightarrow W \rightarrow X \rightarrow \Sigma(Z)$ in $\mathcal{C}^{\text {b }}$. Then $\mathrm{H}(Z)$ is the injective envelope of a (finitely presented) simple functor $S$ by Theorem 8.10. Hence $\mathrm{H}(\mathrm{E})$ contains a simple subfunctor $S$ which clearly is a simple subfunctor of $F$.

The equivalences (ii) $\Leftrightarrow$ (iii) $\Leftrightarrow$ (iv) follow from Corollary 8.11.

COROLLARY 10.3. If $\mathcal{C}$ is of finite type, then $\mathcal{C}^{\mathrm{b}}$ has AR-triangles. 
EXAMPLE 10.4. Let $\Lambda$ be ring which is derived equivalent to a representation finite right hereditary ring, e.g. an Artin algebra which is derived equivalent to the path algebra of Dynkin quiver. Then by [18, Corollary $12.16]$ the unbounded derived category $\mathbf{D}(\operatorname{Mod}-\Lambda)$ is of finite type, so the category of perfect complexes over $\Lambda$ admits AR-triangles. The artinian case also follows from the results of Happel [36].

The following example shows that there exists a compactly generated (pure-semisimple) triangulated category $\mathcal{C}$ such that $\mathcal{C}^{\mathrm{b}}$ is a Krull-Schmidt category with right AR-triangles but with no left AR-triangles.

EXAMPLE 10.5. Consider $\aleph_{t}, t \geqslant 0$, as a totally ordered set and let $k$ be a countable field. Let $\left[\aleph_{t}\right.$, Mod- $\left.k\right]$ be the category of $k$-linear representations of $\aleph_{t}$, and let $k \aleph_{t}$ be the $k$-linear category yielding an equivalence: $\left[\aleph_{t}\right.$, Mod- $\left.k\right] \stackrel{\approx}{\rightarrow}$ Mod- $k \aleph_{t}^{\text {op }}$. If $\mathcal{C}:=\mathbf{D}\left(\operatorname{Mod}-k \aleph_{t}^{\mathrm{op}}\right)$ is the unbounded derived category of Mod- $k \aleph_{t}^{\text {op }}$, then $\mathcal{C}$ is a pure-semisimple compactly generated triangulated category which is not locally finite by Example 12.19 of [18]. Hence, by Theorem 10.2, $\mathcal{C}^{b}$ has right AR-triangles, but no left AR-triangles. It follows that there exist compactly generated triangulated categories $\mathcal{C}$ with the property that $\operatorname{Max}(\mathcal{C}) \subseteq \operatorname{Fin}(\mathcal{C})$ but $\operatorname{Fin}(\mathcal{C}) \nsubseteq \operatorname{Max}(\mathcal{C})$.

\subsection{ENDOFINITE OBJECTS}

Recall from Corollary 9.6 that endofinite objects provide an important class of pure-injective objects of $\mathcal{C}$. In this subsection we study the connections between endofiniteness and the finite type property. Recall that an object $G$ in Mod- $\mathcal{C}^{\text {b }}$, resp. Flat $\left(\mathcal{C}^{\mathrm{b}}\right)$, is called endofinite if the right $\operatorname{End}(G)$-module $(F, G)$ has finite length for any finitely presented object $F$ in $\operatorname{Mod}-\mathcal{C}^{b}, \operatorname{resp} . \operatorname{Flat}\left(\mathcal{C}^{b}\right)$, see [27]. We begin our discussion of endofinite objects with the following.

LEMMA 10.6. For an object $E$ in $\mathcal{C}$, the following are equivalent.

(i) $E$ is endofinite in $\mathcal{C}$.

(ii) $\mathrm{H}(E)$ is endofinite in Mod- $\mathcal{C}^{\mathrm{b}}$.

(iii) $\mathrm{H}(E)$ is endofinite in $\operatorname{Flat}\left(\mathcal{C}^{\mathrm{b}}\right)$.

The functor $\stackrel{\mathcal{C}}{\rightarrow} \rightarrow$ Mod- $\mathcal{C}^{\mathrm{b}}$ gives equivalences between the subcategories of endofinite objects of $\mathcal{C}$, endofinite objects of $\operatorname{Flat}\left(\mathcal{C}^{b}\right)$ and endofinite flat objects of Mod- $\mathcal{C}^{\mathrm{b}}$.

Proof. Assume that $E$ is endofinite. Then we have a ring isomorphism $\operatorname{End}_{\mathcal{C}}(E) \cong \operatorname{End}(\mathrm{H}(E))$ since $E$ is pure-injective. Let $F$ be a finitely presented functor over $\mathcal{C}^{\mathrm{b}}$ and let $\mathrm{H}(Y) \rightarrow \mathrm{H}(X) \rightarrow F \rightarrow 0$ be a finite presentation of $F$. Then we have the exact sequence $0 \rightarrow[F, \mathrm{H}(E)] \rightarrow \mathcal{C}(X, E) \rightarrow \mathcal{C}(Y, E)$ of right

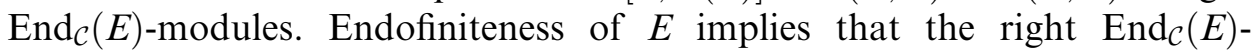


modules $\mathcal{C}(X, E)$ and $\mathcal{C}(Y, E)$ have finite length. Then obviously the right $\operatorname{End}_{\mathcal{C}}(E)$-module $[F, \mathrm{H}(E)]$ has finite length, so $\mathrm{H}(E)$ is endofinite in Mod- $\mathcal{C}^{\text {b }}$. Conversely if $\mathrm{H}(E)$ is endofinite in Mod- $\mathcal{C}^{\mathrm{b}}$, then $\mathrm{H}(E)$ is pure-injective. Since $\mathrm{H}(E)$ is also flat, it follows that $\mathrm{H}(E)$ is injective. Thus $E$ is pure-injective in $\mathcal{C}$ and in particular the natural ring map $\operatorname{End}_{\mathcal{C}}(E) \rightarrow \operatorname{End}(\mathrm{H}(E))$ is invertible. Since for any compact object $X$ in $\mathcal{C}$, the functor $\mathrm{H}(X)$ is finitely presented, it follows that the right $\operatorname{End}_{\mathcal{C}}(E)$-module $\mathcal{C}(X, E) \stackrel{\cong}{\rightarrow}[\mathrm{H}(X), \mathrm{H}(E)]$ has finite length. So $E$ is endofinite in $\mathcal{C}$. The proof of the equivalence (i) $\Leftrightarrow$ (iii) is similar and is left to the reader. The last assertion follows from the fact that $\mathrm{H}$ induces an equivalence between the full subcategory of pure-injective objects of $\mathcal{C}$ and the flat and pure-injective objects of Mod- $\mathcal{C}^{\mathrm{b}}$.

As a direct consequence of Lemma 10.6, the results of the previous sections and the results of Prest [71, Proposition 2.11] and Krause in [53, Proposition 6.17] we have the following corollary.

COROLLARY 10.7. Let $E$ be an indecomposable endofinite object in $\mathcal{C}$. Then $\{E\}$ is a closed point in $\mathrm{Zg}(\mathcal{C})$. In particular if any compact object is endofinite, then $\mathcal{C}^{\mathrm{b}}$ has left AR-triangles iff any indecomposable compact object is clopen in $\mathrm{Zg}(\mathcal{C})$.

EXAMPLE 10.8. Let $\mathcal{C}$ be the stable homotopy category of $p$-local spectra, where $p$ is a prime, see [61, Chapter 8]. It is known that the Morava $K$ theories $K(n), n \geq 0$ are indecomposable endofinite objects in $\mathcal{C}$, see [55]. Hence each $K(n)$ is a closed point in the Ziegler spectrum of $\mathcal{C}$.

There is a handy characterization of endofiniteness in terms of certain quotient categories of Mod- $\mathcal{C}^{\mathrm{b}}$. Let $E$ be an object in $\mathcal{C}$ and consider the functor $\mathrm{H}(E)$ in Mod- $\mathcal{C}^{\mathrm{b}}$. Let $\mathcal{S}_{E}$ be the full subcategory of mod- $\mathcal{C}^{\mathrm{b}}$ consisting of all functors $F$ such that $[F, \mathrm{H}(E)]=0$. Let $\mathcal{L}_{E}:=\lim \mathcal{S}_{E}$ be the full subcategory of Mod- $\mathcal{C}^{\mathrm{b}}$ consisting of all functors $G$ which can be written as a filtered colimit of objects from $\mathcal{S}_{E}$. Since $\mathrm{H}(E)$ is flat it is easy to see that $\mathcal{S}_{E}$ is a Serre subcategory of mod- $\mathcal{C}^{\mathrm{b}}$ and therefore $\mathcal{L}_{E}$ is a localizing subcategory of Mod- $\mathcal{C}^{\mathrm{b}}$. The following criterion follows from the results of Herzog [41, Proposition 7.6] or Krause [50, Theorem 1.2].

LEMMA 10.9. The object $E$ is endofinite in $\mathcal{C}$ iff $\operatorname{Mod}-\mathcal{C}^{\mathrm{b}} / \mathcal{L}_{E}$ is locally finite.

We are interested in having criteria ensuring that the maximal points of the Ziegler spectrum are endofinite. In this connection we have the following.

LEMMA 10.10. Let $X$ be a compact object with local endomorphism ring. Then the $\mathrm{m}$-dual object $\mathbb{D}_{\mathfrak{m}}(X)$ is endofinite iff for any compact object $Y$ in $\mathcal{C}$, the left $\Lambda_{X}$-module $\mathcal{C}^{\mathrm{b}}(X, Y)$ has finite length. In particular $\Lambda_{X}$ is left Artinian. 
Proof. We have isomorphisms $\mathcal{C}\left(Y, \mathbb{D}_{\mathfrak{m}}(X)\right) \cong \operatorname{Hom}_{\Lambda_{X}}\left[\mathcal{C}(X, Y), I_{\mathfrak{m}}\right]$ and $\operatorname{End}_{\Lambda_{X}}\left(I_{\mathfrak{m}}\right) \cong \operatorname{End}_{\mathcal{C}}\left(\mathbb{D}_{\mathfrak{m}}(X)\right)$. Since $I_{\mathfrak{m}}$ is an injective cogenerator in Mod- $\Lambda_{X}^{\mathrm{op}}$, it follows that $\mathbb{D}_{\mathfrak{m}}(X)$ is endofinite iff the left $\Lambda_{X}$-module $\mathcal{C}(X, Y)$ has finite length.

COROLLARY 10.11. Let $\mathcal{C}$ be a compactly generated $R$-linear triangulated category over a commutative Artin ring $R$. If $\mathcal{C}^{\mathrm{b}}$ is compactly $R$-finite, then for any indecomposable compact object $X$, the $\mathrm{m}$-dual object $\mathbb{D}_{\mathfrak{m}}(X)$ is endofinite and $\left\{\mathbb{D}_{\mathfrak{m}}(X)\right\}$ is a closed subset of the Ziegler spectrum $\mathrm{Zg}(\mathcal{C})$. Moreover we have the following.

(i) $X$ is the target of an AR-triangle in $\mathcal{C}^{\mathrm{b}}$ iff $\left\{\mathbb{D}_{\mathfrak{m}}(X)\right\}$ is clopen in $\mathrm{Zg}(\mathcal{C})$.

(ii) $\mathcal{C}^{\mathrm{b}}$ has right AR-triangles iff $\left\{\tau^{+}(X)\right\}$ is clopen in $\operatorname{Zg}(\mathcal{C}), \forall X \in \operatorname{lnd}\left(\mathcal{C}^{\mathrm{b}}\right)$.

(iii) If $\mathcal{C}^{\mathrm{b}}$ has AR-triangles, then any compact object is endofinite and the set $\{X\}$ is clopen in $\mathrm{Zg}(\mathcal{C})$ for any $X \in \operatorname{Ind}\left(\mathcal{C}^{\mathrm{b}}\right)$.

Proof. Since $\mathcal{C}(X, Y)$ is finitely generated as an $R$-module and $\Lambda_{X}$ is an Artin $R$-algebra, it follows that $\mathcal{C}(X, Y)$ has finite length as a left $\Lambda_{X}$-module. Then the assertions follow from Lemma 10.10 and Corollary 10.7. Now the proof of parts (i) and (ii) follow from Theorem 8.12 and the proof of part (iii) follows from the fact that any compact object is of the form $\mathbb{D}_{\mathfrak{m}}(X)$.

In the sequel we shall need the following result of Auslander [2, Theorem 2.12].

LEMMA 10.12. If $\mathcal{C}^{\mathrm{b}}$ is Krull-Schmidt and $E$ is in $\mathcal{C}$, then $\mathrm{H}(E)$ has finite length in Mod- $\mathcal{C}^{\mathrm{b}}$ iff for any indecomposable compact object $X$, the left $\Lambda_{X^{-}}$ module $\mathcal{C}(X, E)$ has finite length and $\mathcal{C}(Y, E)=0$ for almost all indecomposable compact objects $Y$.

Recall that an associative ring $\Lambda$ is representation finite iff any left or right $\Lambda$-module is endofinite, see [68], [83] or [53]. We have the following analogous result which gives a connection between the finite type property and endofiniteness in compactly generated triangulated categories.

THEOREM 10.13. For a compactly generated triangulated category $\mathcal{C}$ the following statements are equivalent.

(i) $\mathcal{C}$ is of finite type.

(ii) Any object of $\mathcal{C}$ is endofinite.

(iii) Any compact object of $\mathcal{C}$ is endofinite and for any compact object $X$ in $\mathcal{C}$ we have $\left|\operatorname{SuppC}^{\mathrm{b}}(X,-)\right|<\infty$.

(iv) Any compact object of $\mathcal{C}$ is endofinite and for any compact object $X$ in $\mathcal{C}$ we have $\left|\operatorname{Supp}^{\mathrm{b}}(-, X)\right|<\infty$.

Proof. (i) $\Rightarrow$ (ii) Since $\mathcal{C}$ is of finite type, the category Mod- $\mathcal{C}^{\text {b }}$ is locally finite. If $E$ is an object in $\mathcal{C}$, then Mod- $\mathcal{C}^{\mathrm{b}} / \mathcal{L}_{E}$ is locally finite as a Gabriel 
quotient of the locally finite category Mod- $\mathcal{C}^{b}$. Hence $E$ is endofinite, by Lemma 10.9.

(ii) $\Rightarrow$ (i) By Lemma 10.9 , for any object $C \in \mathcal{C}$, the category Mod-C $\mathcal{C}^{\mathrm{b}} / \mathcal{L}_{C}$ is locally finite. In particular the pure-injective cogenerator $E:=\prod\left\{\mathbb{D}_{\mathfrak{m}}(X) \mid X \in \operatorname{Iso}\left(\mathcal{C}^{\mathrm{b}}\right), \mathfrak{m} \in \operatorname{Max}_{l}\left(\Lambda_{X}\right)\right\}$ of $\mathcal{C}$ is endofinite. Since $\mathrm{H}(E)$ is an injective cogenerator in Mod- $\mathcal{C}^{\mathrm{b}}$, it follows that $\mathcal{L}_{E}=0$ and therefore Mod- $\mathcal{C}^{b}$ is locally finite. Hence $\mathcal{C}$ is of finite type.

(i) $\Leftrightarrow$ (iii), (iv) If $\mathcal{C}$ is of finite type then, by (ii), any compact object is endofinite. By [18, Proposition 11.23], Mod- $\left\{\mathcal{C}^{b}\right\}^{\text {op }}$ is locally finite and therefore $\left|\operatorname{Supp}^{\mathrm{b}}(X,-)\right|<\infty$ by Lemma 10.12. Conversely if (iii) holds then for any compact object $X$, the endomorphism $\operatorname{ring} \operatorname{End}_{\mathcal{C}}(X)$ is left Artinian. This implies that $\mathcal{C}^{\mathrm{b}}$ is Krull-Schmidt. Then by Lemma 10.12 and [18] we infer that Mod- $\mathcal{C}^{\text {b }}$ is locally finite, so $\mathcal{C}$ is of finite type. The equivalence (i) $\Leftrightarrow$ (iv) follows similarly.

We have the following consequence which gives in particular a triangulated analogue to a well-known module theoretic result of Auslander, see [3, Theorem A'].

COROLLARY 10.14. Let $\mathcal{C}$ be an R-linear triangulated category over a commutative Artin ring $R$. Then $\mathcal{C}$ is compactly $R$-finite iff $\mathcal{C}^{b}$ is Krull-Schmidt and any maximal point $\mathbb{D}_{\mathfrak{m}}(X)$ of $\mathcal{C}$ is endofinite. If this is the case, then the following are equivalent.

(i) $\mathcal{C}$ is of finite type.

(ii) $\mathcal{C}$ is pure-semisimple.

Proof. If $\mathcal{C}$ is compactly $R$-finite, then $\mathcal{C}^{\mathrm{b}}$ is Krull-Schmidt and then Lemma 10.10 implies that any maximal point $\mathbb{D}_{\mathfrak{m}}(X)$ of $\mathcal{C}$ is endofinite. Conversely if this happens, by Lemma 10.10 the endomorphism ring $\Lambda_{X}$ of any compact object $X$ is left Artinian and therefore $\mathcal{C}$ is compactly $R$-finite. Now let $\mathcal{C}$ be pure-semisimple and compactly $R$-finite. Then any compact object is endofinite and any maximal point $\mathbb{D}_{\mathfrak{m}}(X)$ is compact. Since, by $[26$, Proposition 4.3], the class of endofinite objects is closed under products, the pure-injective cogenerator of $\mathcal{C}$ is endofinite. This implies that Mod- $\mathcal{C}^{\mathrm{b}}$ is locally finite and consequently $\mathcal{C}$ is of finite type.

\section{Compactly Dual Pairs of Triangulated Categories}

It is well-known that the dual of a compactly generated triangulated category is never compactly generated, see [66, Appendix E]. However there are important examples of pairs of compactly generated triangulated categories such that the subcategories of compact objects are connected by an exact duality. In this section we study such pairs in connection with the structure of their Ziegler spectra and the behavior of Auslander-Reiten triangles. 
Throughout we fix a compactly generated triangulated category $\mathcal{C}$. There is a close connection between endofinite compact objects in $\mathcal{C}$ and the pure global dimensions attached to $\mathcal{C}$. Recall from [18] that the pure global dimension p.gl.dim $\mathcal{C}$ of $\mathcal{C}$ is defined as the supremum of the projective dimensions of the flat functors $\left\{\mathcal{C}^{\mathrm{b}}\right\}^{\mathrm{op}} \rightarrow \mathcal{A} b$ and the dual pure global dimension p.gl.dim $\mathcal{C}^{\text {op }}$ of $\mathcal{C}$ is defined to be the supremum of the projective dimensions of the flat functors $\mathcal{C}^{\mathrm{b}} \rightarrow \mathcal{A} b$. By [18, Proposition 11.23], $\mathcal{C}$ is puresemisimple iff p.gl.dimC $=0$, and $\mathcal{C}$ is of finite type iff p.gl.dim $\mathcal{C}^{\text {op }}=$ $0=$ p.gl.dimC . Note that p.gl.dim $\mathcal{C}$ is not the pure global dimension of $\mathcal{C}^{\text {op }}$ since the latter is never compactly generated. However the definition of dual pure global dimension is reasonable because of the following.

Remark 11.1. [18, Section 11.6] Let $\mathcal{C}$ and $\mathcal{D}$ be compactly generated triangulated categories and assume that there exists an (exact) duality $\left\{\mathcal{C}^{\mathrm{b}}\right\}^{\mathrm{op}} \stackrel{\approx}{\longrightarrow} \mathcal{D}^{\mathrm{b}}$. Then p.gl.dim $\mathcal{C}^{\mathrm{op}}=$ p.gl.dim $\mathcal{D}$. Instances of this situation are the following.

(i) Let $\Lambda$ be an associative ring. Then there is a duality between $\mathbf{D}(\operatorname{Mod}-\Lambda)^{\text {b }}$ and $\mathbf{D}\left(\operatorname{Mod}-\Lambda^{\text {op }}\right)^{\mathrm{b}}$ which is induced by the duality between the categories of finitely generated projective left and right modules. We infer that p.gl.dimD $(\operatorname{Mod}-\Lambda)^{\mathrm{op}}=$ p.gl.dim $\mathbf{D}\left(\operatorname{Mod}-\Lambda^{\mathrm{op}}\right)$.

(ii) Let $\mathrm{Ho}(\mathcal{S} p)$ be the stable homotopy category of spectra. Then SpanierWhitehead duality gives a self duality on the category of finite spectra which is the full subcategory of compact objects of $\mathrm{Ho}(\mathcal{S} p)$. In this case p.gl.dim $\mathrm{Ho}(\mathcal{S} p)^{\mathrm{op}}=$ p.gl.dim $\mathrm{Ho}(\mathcal{S} p)$ which is equal to 1 by [18].

(iii) Let $\Lambda$ be a QF-ring. Since the stable categories mod- $\Lambda^{\text {op }}$ and $\bmod -\Lambda$ are dual, we infer that p.gl.dim $(\underline{\operatorname{Mod}}-\Lambda)^{\mathrm{op}}=$ p.gl.dimMod- $\Lambda^{\mathrm{op}}$.

We have the following result which is a triangulated analogue of a module theoretic result of Herzog, see [40, Theorem 2.3], proved via model theoretic methods. First we recall that an object $E$ in $\mathcal{C}$ is called $\Sigma$-pure-injective provided that any coproduct of copies of $E$ is pure-injective.

THEOREM 11.2. If $\mathcal{C}$ has dual pure global dimension zero, then any compact object of $\mathcal{C}$ is endofinite. Moreover $\mathcal{C}^{\mathrm{b}}$ has left AR-triangles which are $A R$ triangles in $\mathcal{C}$ and any compact object of $\mathcal{C}$ is $\Sigma$-pure-injective.

Proof. By [18, Proposition 11.23] the hypothesis implies that the category $\mathcal{C}^{b}$ is Krull-Schmidt and the functor category Mod- $\mathcal{C}^{\mathrm{b}}$ is locally Artinian. Let $X$ be a compact object. Since Mod- $\mathcal{C}^{\mathrm{b}}$ is locally Artinian, it follows that the functor $\mathrm{H}(X)$ is Artinian. Viewing $\mathrm{H}(X)$ as a finitely presented object in the locally finitely presented category $\operatorname{Flat}\left(\mathcal{C}^{\mathrm{b}}\right)$, it follows that for any compact object $Y$, the space $\mathcal{C}^{\mathrm{b}}(Y, X)$ satisfies the descending chain condition for finite matrix subgroups in the sense of [27]. Hence by [27, Theorem 3.5.1] we have 
that $\mathrm{H}(X)$ is $\Sigma$-pure-injective in $\operatorname{Flat}\left(\mathcal{C}^{\mathrm{b}}\right)$. This clearly implies that $X$ is $\Sigma$ pure-injective in $\mathcal{C}$. Hence any compact object in $\mathcal{C}$ is $\Sigma$-pure-injective. By the Krull-Schmidt property, to show that any compact object is endofinite it suffices to show that this holds for any indecomposable compact object. Hence we can assume that $X$ is indecomposable. Then by the above observations, $X$ is a point in the Ziegler spectrum. Since $X$ is $\Sigma$-pure-injective, as in [53, Proposition 6.17], it suffices to show that $\{X\}$ is closed in $\operatorname{Zg}(\mathcal{C})$. Let $W$ be indecomposable in $\mathcal{C}^{\mathrm{b}}$. Since Mod- $\mathcal{C}^{\mathrm{b}}$ is locally Artinian, the functor $\mathrm{H}(W)$ is Artinian and therefore $\mathrm{H}(X)$ contains a simple subfunctor $S$. Then Theorem 8.10 implies that there exists an AR-triangle $W \stackrel{\phi}{\rightarrow} Y \rightarrow Z \rightarrow \Sigma(W)$ in $\mathcal{C}^{\text {b }}$. Clearly any morphism $W \rightarrow X$ factors through $\phi$ and consequently $\{X\}=\mathbf{U}_{\phi}$ is a closed point of $\operatorname{Zg}(\mathcal{C})$. We infer that $X$ is endofinite and it remains to show that any AR-triangle in $\mathcal{C}^{\mathrm{b}}$ remains an AR-triangle in $\mathcal{C}$. Since any compact object is pure-injective and $\mathcal{C}^{\mathrm{b}}$ has left AR-triangles, this follows from Corollary 9.2.

Summarizing the above results we have the following direct consequences.

COROLLARY 11.3. Let p.gl.dim $\mathcal{C}^{\text {op }}=0$ and let $E$ be a point in $\mathrm{Zg}(\mathcal{C})$. Then $E$ is compact iff $\{E\}$ is clopen iff $E$ is endofinite and a maximal point in $\mathrm{Zg}(\mathcal{C})$.

COROLLARY 11.4. If $\mathcal{C}$ is compactly generated, then the following are equivalent.

(i) $\mathcal{C}$ is of finite type.

(ii) p.gl.dim $\mathcal{C}^{\mathrm{op}}=0$ and $\mathcal{C}^{\mathrm{b}}$ has right $A R$-triangles.

(iii) p.gl.dim $\mathcal{C}=0$ and $\mathcal{C}^{\mathrm{b}}$ has left $A R$-triangles.

(iv) p.gl.dim $\mathcal{C}^{\mathrm{op}}=0$ and for any indecomposable compact object $X$ in $\mathcal{C}$ we have: $\left|\operatorname{Supp}^{\mathrm{b}}(X,-)\right|<\infty$ or $\left|\operatorname{Supp}^{\mathrm{b}}(-, X)\right|<\infty$.

Formalizing Remark 11.1, we say that two compactly generated triangulated categories $\mathcal{C}$ and $\mathcal{D}$ are compactly dual if there exists an (exact) duality $\mathrm{D}:\left\{\mathcal{C}^{\mathrm{b}}\right\}^{\mathrm{op}} \stackrel{\approx}{\rightarrow} \mathcal{D}^{\mathrm{b}}$. This notion gives a triangulated analogue to the well-known duality $\bmod -(\bmod -\Lambda)\}^{\text {op }} \underset{\rightarrow}{\sim} \bmod -\left(\bmod -\Lambda^{\text {op }}\right)^{\text {op }}$, where $\Lambda$ is a ring, discovered by Auslander [12] and Gruson-Jensen [34]. Part (iv) of the next result, also observed by Garkusha-Prest [32], gives a triangulated analogue to a result of Herzog [41, Theorem 5.5].

THEOREM 11.5. Let $(\mathcal{D}, \mathcal{C})$ be a pair of compactly dual compactly generated triangulated categories and let $\mathrm{D}:\left\{\mathcal{C}^{\mathrm{b}}\right\}^{\mathrm{op}} \stackrel{\approx}{\rightarrow} \mathcal{D}^{\mathrm{b}}$ be an exact duality.

(i) $\mathcal{C}^{\mathrm{b}}$ has right (left) AR-triangles iff $\mathcal{D}^{\mathrm{b}}$ has left (right) AR-triangles.

(ii) $\operatorname{Max}_{\text {Ind }}(\mathcal{C}) \subseteq \operatorname{Fin}(\mathcal{C})$ iff $\operatorname{Fin}(\mathcal{D}) \subseteq \operatorname{Max}_{\operatorname{lnd}}(\mathcal{D})$ and $\operatorname{Fin}(\mathcal{C}) \subseteq \operatorname{Max}_{\operatorname{lnd}}(\mathcal{C})$ iff $\operatorname{Max}_{\operatorname{lnd}}(\mathcal{D}) \subseteq \operatorname{Fin}(\mathcal{D})$.

(iii) $\mathcal{C}$ is of finite type iff $\mathcal{D}$ is of finite type. 
(iv) The frames of open subsets of the Ziegler spectra $\operatorname{Zg}(\mathcal{C})$ and $\operatorname{Zg}(\mathcal{D})$ are isomorphic.

(v) If $\mathcal{D}$ is pure semisimple, then the duality $\mathrm{D}$ induces a bijection

$$
\operatorname{Ind}\left(\mathcal{C}^{\mathrm{b}}\right) \longrightarrow \operatorname{Isol}(\mathcal{D}), X \mapsto \mathbb{D}_{\mathfrak{m}}(\mathrm{D}(X)) \text {. }
$$

(vi) If $\mathcal{D}$ is pure semisimple, then any compact object in $\mathcal{C}$ is endofinite and any isolated point, in particular any maximal point, in $\operatorname{Zg}(\mathcal{D})$ is endofinite.

Proof. Parts (i), (ii) and (iii) follow directly from our previous results and part (iv) follows as in [41, Theorem 5.5] by using [55]. Assume now that $\mathcal{D}$ is pure-semisimple. Then by Lemma 10.1 the category $\mathcal{D}^{\mathrm{b}}$, hence also $\mathcal{C}^{\mathrm{b}}$, is Krull-Schmidt.

(v) Let $X$ be an indecomposable compact object in $\mathcal{C}$. Then $\mathrm{D}(X)$ is an indecomposable compact object in $\mathcal{D}$. Let $\Sigma^{-1} \mathbb{D}_{\mathfrak{m}}(\mathrm{D}(X)) \rightarrow A \rightarrow \mathrm{D}(X) \rightarrow$ $\mathbb{D}_{\mathfrak{m}}(\mathrm{D}(X))$ be an AR-triangle in $\mathcal{D}$. Since $\mathcal{D}$ is pure semisimple, any compact object is pure injective and therefore the object $\mathbb{D}_{\mathfrak{m}}(\mathrm{D}(X))$ is compact and the above triangle is an AR-triangle in $\mathcal{D}^{\mathrm{b}}$. Then by Corollary 7.8 , the object $\mathbb{D}_{\mathfrak{m}}(\mathrm{D}(X))$ is isolated. Using the uniqueness of AR-triangles, we infer that the map $X \mapsto \mathbb{D}_{\mathfrak{m}}(\mathrm{D}(X))$ is an injection. If $E$ is an isolated point in $\mathrm{Zg}(\mathcal{D})$, then by Corollary 7.7 it follows that $E$ is a maximal point, hence of the form $\mathbb{D}_{\mathfrak{m}}(Y)$ for some indecomposable compact object $Y$ in $\mathcal{D}$. In turn $Y \cong \mathrm{D}(X)$ where $X$ is an indecomposable compact object in $\mathcal{C}$. Hence $E \cong \mathbb{D}_{\mathfrak{m}}(\mathrm{D}(X))$ and therefore the map $X \mapsto \mathbb{D}_{\mathfrak{m}}(\mathrm{D}(X))$ is a bijection.

(vi) The first part is Theorem 11.2. By part (v) it suffices to show that the object $\mathbb{D}_{\mathfrak{m}}(\mathrm{D}(X))$ is endofinite, for any indecomposable compact object $X$ in $\mathcal{C}$. Let $Z$ be a compact object in $\mathcal{D}$. Then we have isomorphisms

$$
\mathcal{D}\left(Z, \mathbb{D}_{\mathfrak{m}}(\mathrm{D}(X))\right) \stackrel{\cong}{\rightrightarrows}\left[\mathcal{D}(\mathrm{D}(X), Z), I_{\mathfrak{m}}\right] \stackrel{\cong}{\rightarrow}\left[\mathcal{C}(\mathrm{D}(Z), X), I_{\mathfrak{m}}\right]
$$

which show that the length of the right $\operatorname{End}\left(\mathbb{D}_{\mathfrak{m}}(\mathrm{D}(X))\right.$-module $\mathcal{D}\left(Z, \mathbb{D}_{\mathfrak{m}}(\mathrm{D}(X))\right)$ is equal to the length of the right $\operatorname{End}\left(I_{\mathfrak{m}}\right)$-module $\left[\mathcal{C}(\mathrm{D}(Z), X), I_{\mathfrak{m}}\right]$ because $\operatorname{End}\left(I_{\mathfrak{m}}\right) \cong \operatorname{End}\left(\mathbb{D}_{\mathfrak{m}}(\mathrm{D}(X))\right.$. Since $I_{\mathfrak{m}}$ is an injective cogenerator for the category of $\operatorname{End}(X)$-modules, it follows that the length of the right $\operatorname{End}\left(I_{\mathfrak{m}}\right)$-module $\mathcal{D}\left(Z, \mathbb{D}_{\mathfrak{m}}(\mathrm{D}(X))\right)$ is equal to the length of the right End $(X)$-module $\mathcal{C}(\mathrm{D}(Z), X)$ which is finite, since the object $\mathrm{D}(Z)$ is compact in $\mathcal{C}$ and the compact object $X$ is endofinite.

\section{Auslander-Reiten Triangles and Grothendieck Groups}

For Artin algebras there is an interesting relationship between AR-sequences and Grothendieck groups in connection with the representation type which is due to Auslander and Butler, see [13]. In this section we show that this relationship holds more generally in a compactly generated triangulated category. 
Recall that the Grothendieck group $\mathrm{K}_{0}(\mathcal{T})$ of a skeletally small triangulated category $\mathcal{T}$ is defined to be the quotient of the free abelian group on the set $\{(X) \mid X \in \mathcal{T}\}$ of isoclasses of objects of $\mathcal{T}$ modulo the subgroup generated by all elements of the form $(X)-(Y)+(Z)$ where $X \rightarrow Y \rightarrow Z \rightarrow \Sigma(X)$ is a triangle in $\mathcal{T}$. Let $\mathrm{K}_{0}(\mathcal{T}, \oplus)$ be the Grothendieck group of the monoidal category $(\mathcal{T}, \oplus)$ and let $[X]$ be the isoclass of the object $X$ in $\mathrm{K}_{0}(\mathcal{T})$ or $\mathrm{K}_{0}(\mathcal{T}, \oplus)$. There is a natural surjection $\mathrm{K}_{0}(\mathcal{T}, \oplus) \rightarrow \mathrm{K}_{0}(\mathcal{T})$ which induces a short exact sequence of abelian groups

$$
0 \longrightarrow \Delta(\mathcal{T}) \longrightarrow \mathrm{K}_{0}(\mathcal{T}, \oplus) \longrightarrow \mathrm{K}_{0}(\mathcal{T}) \longrightarrow 0
$$

Let $\operatorname{art}(\mathcal{T})$ be the set of all elements in $\mathrm{K}_{0}(\mathcal{T}, \oplus)$ of the form $[X]-[Y]+[Z]$ where $X \rightarrow Y \rightarrow Z \rightarrow \Sigma(X)$ is an AR-triangle in $\mathcal{T}$, and let $\operatorname{ART}(\mathcal{T})$ be the subgroup of $\mathrm{K}_{0}(\mathcal{T}, \oplus)$ generated by $\operatorname{art}(\mathcal{T})$. Note that if $\mathcal{T}$ is Krull-Schmidt, then $\mathrm{K}_{0}(\mathcal{T}, \oplus)$ is free on the set of isoclasses of indecomposable objects of $\mathcal{T}$.

The following result generalizes the well-known characterization of representation-finite Artin algebras in terms of Grothendieck groups, due to Auslander-Butler, see [13, Theorem VI.4.3].

THEOREM 12.1 Let $\mathcal{C}$ be a compactly generated triangulated category. Then for the following statements

(i) $\mathcal{C}$ is of finite type.

(ii) $\mathcal{C}^{\mathrm{b}}$ is a Krull-Schmidt category with $A R$-triangles and $\Delta\left(\mathcal{C}^{\mathrm{b}}\right)$ is generated by the set $\operatorname{art}\left(\mathcal{C}^{\mathrm{b}}\right)$.

we have (i) $\Rightarrow$ (ii). If $\mathcal{C}$ is compactly $R$-finite over a commutative ring $R$, then (ii) $\Rightarrow(\mathrm{i}), \operatorname{ART}\left(\mathcal{C}^{\mathrm{b}}\right)=\Delta\left(\mathcal{C}^{\mathrm{b}}\right)$ and the set $\operatorname{art}\left(\mathcal{C}^{\mathrm{b}}\right)$ is a free basis of $\Delta\left(\mathcal{C}^{\mathrm{b}}\right)$.

Proof. (i) $\Rightarrow$ (ii) Since $\mathcal{C}$ is of finite type, it follows that the functor category Mod $-\mathcal{C}^{b}$ is locally finite and therefore mod- $\mathcal{C}^{b}$ is the full subcategory of finite length objects. By Lemma 10.1 and Corollary 10.3, the category $\mathcal{C}^{\text {b }}$ is Krull-Schmidt with AR-triangles. It suffices to show that any element $[X]-[Y]+[Z]$ in $\mathrm{K}_{0}\left(\mathcal{C}^{\mathrm{b}}, \oplus\right)$ arising from a triangle $X \stackrel{g}{\rightarrow} Y \stackrel{f}{\rightarrow} Z \stackrel{h}{\rightarrow} \Sigma(X)$ in $\mathcal{C}^{\mathrm{b}}$ is a finite sum of elements $\left[X_{i}\right]-\left[Y_{i}\right]+\left[Z_{i}\right]$ arising from AR-triangles $X_{i} \rightarrow Y_{i} \rightarrow Z_{i} \rightarrow \Sigma\left(X_{i}\right)$ in $\mathcal{C}^{\mathrm{b}}$. Consider the exact sequence $0 \rightarrow F \rightarrow$ $\mathrm{H}(X) \stackrel{\mathrm{H}(g)}{\rightarrow} \mathrm{H}(Y) \stackrel{\mathrm{H}(f)}{\rightarrow} \mathrm{H}(Z) \rightarrow F^{*} \rightarrow 0$ in mod- $\mathcal{C}^{\mathrm{b}}$. Then $F=\operatorname{Coker}^{\mathrm{b}}\left(-, \Sigma^{-1}(f)\right)$ and $F^{*}=\operatorname{Coker}^{\mathrm{b}}(-, f)$, in particular $F=F^{*} \Sigma$. Then the above exact sequence shows that in $\mathrm{K}_{0}\left(\bmod -\mathcal{C}^{\mathrm{b}}\right)$ we have the relation:

$$
[F]+\left[F^{*}\right]=[\mathrm{H}(X)]-[\mathrm{H}(Y)]+[\mathrm{H}(Z)]
$$

Since mod- $\mathcal{C}^{\mathrm{b}}$ is a length category, the functors $F$ and $F^{*}$ have finite length. Hence in the Grothendieck group $\mathrm{K}_{0}\left(\bmod -\mathcal{C}^{\mathrm{b}}\right)$ we have $[F]=\sum_{i=1}^{n} \lambda_{i}\left[S_{i}\right]$ where $S_{i}$ is a simple functor in mod- $\mathcal{C}^{\text {b }}$. Clearly if $0 \subseteq F_{0} \subseteq F_{1} \subseteq$ $F_{2} \subseteq \cdots \subseteq F_{n-1} \subseteq F_{n}=F$ is a composition series of $F$ with composition factors $S_{i}$ each with multiplicity $\lambda_{i}$, then $0 \subseteq F_{0} \Sigma^{-1} \subseteq F_{1} \Sigma^{-1} \subseteq$ 
$F_{2} \Sigma^{-1} \subseteq \cdots \subseteq F_{n-1} \Sigma^{-1} \subseteq F_{n} \Sigma^{-1}=F \Sigma^{-1}=F^{*}$ is a composition series of $F^{*}$ with composition factors $S_{i} \Sigma^{-1}=S_{i}^{*}$ each with multiplicity $\lambda_{i}$. Hence from (1) we obtain the relation

$$
[F]+\left[F^{*}\right]=\sum_{i=1}^{n} \lambda_{i}\left(\left[S_{i}\right]+\left[S_{i}^{*}\right]\right)
$$

Since the simple functor $S_{i}$ is finitely presented, there exists an AR-triangle $X_{i} \rightarrow Y_{i} \rightarrow Z_{i} \rightarrow \Sigma\left(X_{i}\right)$ in $\mathcal{C}^{\mathrm{b}}$, which induces an exact sequence $0 \rightarrow S_{i} \rightarrow \mathrm{H}\left(X_{i}\right) \rightarrow \mathrm{H}\left(Y_{i}\right) \rightarrow \mathrm{H}\left(Z_{i}\right) \rightarrow S_{i}^{*} \rightarrow 0$ in mod- $\mathcal{C}^{\mathrm{b}}$. Hence we have the relations

$$
\left[S_{i}\right]+\left[S_{i}^{*}\right]=\left[\mathrm{H}\left(X_{i}\right)\right]-\left[\mathrm{H}\left(Y_{i}\right)\right]+\left[\mathrm{H}\left(Z_{i}\right)\right]
$$

in $\mathrm{K}_{0}\left(\bmod -\mathcal{C}^{\mathrm{b}}\right)$ and therefore from $(1),(2)$ and (3) we obtain the relation

$$
[\mathrm{H}(X)]-[\mathrm{H}(Y)]+[\mathrm{H}(Z)]=\sum_{i=1}^{n} \lambda_{i}\left(\left[\mathrm{H}\left(X_{i}\right)\right]-\left[\mathrm{H}\left(Y_{i}\right)\right]+\left[\mathrm{H}\left(Z_{i}\right)\right]\right)
$$

Since $\mathcal{C}^{\mathrm{b}}$ is Krull-Schmidt, the canonical map $\mathrm{K}_{0}\left(\mathcal{C}^{\mathrm{b}}, \oplus\right) \rightarrow$ $\mathrm{K}_{0}\left(\operatorname{Proj}\left(\bmod -\mathcal{C}^{\mathrm{b}}\right), \oplus\right)$ induced by $\mathrm{H}$ is invertible. By a result of AuslanderReiten, see [8, Proposition 4.1.2], it follows that the Cartan map $\mathrm{K}_{0}\left(\operatorname{Proj}\left(\bmod -\mathcal{C}^{\mathrm{b}}\right), \oplus\right) \rightarrow \mathrm{K}_{0}\left(\bmod -\mathcal{C}^{\mathrm{b}}\right)$, hence the map $\mathrm{K}_{0}\left(\mathcal{C}^{\mathrm{b}}, \oplus\right) \rightarrow$ $\mathrm{K}_{0}\left(\bmod -\mathcal{C}^{b}\right),[X] \mapsto[\mathrm{H}(X)]$, is a monomorphism. Then from (4) we obtain the following relation in $\mathrm{K}_{0}\left(\mathcal{C}^{\mathrm{b}}, \oplus\right)$ which proves the assertion:

$$
[X]-[Y]+[Z]=\sum_{i=1}^{n} \lambda_{i}\left(\left[X_{i}\right]-\left[Y_{i}\right]+\left[Z_{i}\right]\right)
$$

(ii) $\Rightarrow$ (i) If $\mathcal{C}$ is compactly $R$-finite and (ii) holds, then, by Lemma 4.6, $\mathcal{C}^{\mathrm{b}}(X, Y)$ has finite length over $R, \forall X, Y \in \mathcal{C}^{\mathrm{b}}$. Let $\mathcal{F}$ be the full subcategory of mod- $\mathcal{C}^{\mathrm{b}}$ consisting of all functors of finite length. By a result of AuslanderReiten [8, Proposition 1.3.1], the canonical map $\mathrm{K}_{0}(\mathcal{F}) \rightarrow \mathrm{K}_{0}\left(\bmod -\mathcal{C}^{\mathrm{b}}\right)$ is a monomorphism and it is invertible if and only if $\mathcal{F}=\bmod -\mathcal{C}^{\mathrm{b}}$. Hence to show that $\mathcal{C}$ is of finite type, it suffices to show that the canonical map $\mathrm{K}_{0}(\mathcal{F}) \rightarrow \mathrm{K}_{0}\left(\bmod -\mathcal{C}^{\mathrm{b}}\right)$ is surjective. Let $F=\operatorname{Ker}^{\mathrm{b}}(-, g)$ be in mod- $\mathcal{C}^{\mathrm{b}}$ where $g: X \rightarrow Y$ is a morphism in $\mathcal{C}^{\mathrm{b}}$ and let $X \stackrel{g}{\rightarrow} Y \stackrel{f}{\rightarrow} Z \stackrel{h}{\rightarrow} \Sigma(X)$ be a triangle in $\mathcal{C}^{\mathrm{b}}$. Then as before we have $[F]+\left[F^{*}\right]=[\mathrm{H}(X)]-[\mathrm{H}(Y)]+[\mathrm{H}(Z)]$ in $\mathrm{K}_{0}\left(\bmod -\mathcal{C}^{\mathrm{b}}\right)$, where $F^{*}=F \Sigma^{-1}=\operatorname{Coker}^{\mathrm{b}}(-, f)$, and the element $[X]-[Y]+[Z]$ in $\mathrm{K}_{0}\left(\mathcal{C}^{\mathrm{b}}, \oplus\right)$ lies in $\Delta\left(\mathcal{C}^{\mathrm{b}}\right)$ since it arises from a triangle in $\mathcal{C}^{\mathrm{b}}$. By hypothesis we can write $[X]-[Y]+[Z]=\sum_{i=1}^{n} \lambda_{i}\left(\left[X_{i}\right]-\left[Y_{i}\right]+\left[Z_{i}\right]\right)$ where the element $\left[X_{i}\right]-\left[Y_{i}\right]+\left[Z_{i}\right]$ arises from AR-triangles $X_{i} \rightarrow Y_{i} \rightarrow Z_{i} \rightarrow \Sigma\left(X_{i}\right)$ in $\mathcal{C}^{\mathrm{b}}$. Then we have

$$
\begin{aligned}
{[F]+\left[F^{*}\right] } & =\mathrm{H}(X)]-[\mathrm{H}(Y)]+[\mathrm{H}(Z)] \\
& =\sum_{i=1}^{n} \lambda_{i}\left(\left[\mathrm{H}\left(X_{i}\right)\right]-\left[\mathrm{H}\left(Y_{i}\right)\right]+\left[\mathrm{H}\left(Z_{i}\right)\right]\right)
\end{aligned}
$$


in $\mathrm{K}_{0}\left(\bmod -\mathcal{C}^{\mathrm{b}}\right)$ and as in (3), we have $\left[\mathrm{H}\left(X_{i}\right)\right]-\left[\mathrm{H}\left(Y_{i}\right)\right]+\left[\mathrm{H}\left(Z_{i}\right)\right]=\left[S_{i}\right]+\left[S_{i}^{*}\right]$ for each $i$ where $S_{i}$ and $S_{i}^{*}$ are simple functors. Therefore $[F]+\left[F^{*}\right]=\left[F \oplus F^{*}\right]$ lies in $\mathrm{K}_{0}(\mathcal{F})$. Hence there exists a finitely presented functor $G$ of finite length such that $\left[F \oplus F^{*}\right]=[G]$. It is well-known that this implies that there exist short exact sequences $0 \rightarrow H^{\prime} \rightarrow H_{1} \rightarrow H^{\prime \prime} \rightarrow 0$ and $0 \rightarrow H^{\prime} \rightarrow H_{2} \rightarrow$ $H^{\prime \prime} \rightarrow 0$ such that $F \oplus F^{*} \oplus H_{1} \cong G \oplus H_{2}$. Since $G \oplus H_{2}$ lies in $\mathcal{F}$, we infer that $F$ lies in $\mathcal{F}$. Hence $\mathcal{F}=\bmod -\mathcal{C}^{\mathrm{b}}$ and consequently Mod- $\mathcal{C}^{\mathrm{b}}$ is locally finite, i.e. $\mathcal{C}$ is of finite type.

The last assertion is proved as in [13, Section VI.4] using the bilinear form $<-,->: \mathrm{K}_{0}\left(\mathcal{C}^{\mathrm{b}}, \oplus\right) \times \mathrm{K}_{0}\left(\mathcal{C}^{\mathrm{b}}, \oplus\right) \rightarrow \mathbb{Z}$ defined by $<[X],[Y]>=$ the length of the $R$-module $\mathcal{C}^{\mathrm{b}}(X, Y)$, and is left to the reader.

Remark 12.2. The proof of Theorem 12.1 shows that if $\mathcal{T}$ is a skeletally small triangulated category such that the functor category Mod- $\mathcal{T}$ is locally finite, then the elements $[X]-[Y]+[Z]$ arising from AR-triangles in $\mathcal{T}$, generate the kernel of the canonical epimorphism $\mathrm{K}(\mathcal{T}, \oplus) \rightarrow \mathrm{K}_{0}(\mathcal{T})$. This generalizes, and gives the converse to, a recent result of Xiao and Zhu, see [79, Theorem 2.1].

COROLLARY 12.3. Let $\Lambda$ be a Noetherian R-algebra where $R$ is Artinian or Noetherian complete and local. Then the following are equivalent.

(i) $\mathbf{D}(\operatorname{Mod}-\Lambda)$ is of finite type.

(ii) $\Lambda$ is Artinian, gl. $\operatorname{dim} \Lambda<\infty$ and $\operatorname{art}\left(\mathcal{H}^{\mathrm{b}}\left(\mathcal{P}_{\Lambda}\right)\right)$ is a free basis for the kernel of the Cartan map $\mathrm{K}_{0}\left(\mathcal{H}^{\mathrm{b}}\left(\mathcal{P}_{\Lambda}\right), \oplus\right) \rightarrow \mathrm{K}_{0}\left(\mathbf{D}^{\mathrm{b}}(\bmod -\Lambda)\right)$.

If (i) holds, then $\Lambda$ is of representation finite and $\mathrm{K}_{0}\left(\mathcal{H}^{\mathrm{b}}\left(\mathcal{P}_{\Lambda}\right), \oplus\right) \cong$ $\operatorname{ART}\left(\mathcal{H}^{\mathrm{b}}\left(\mathcal{P}_{\Lambda}\right)\right) \oplus \mathbb{Z}^{n}$, where $n=\operatorname{rankK}_{0}(\Lambda)$ is the number of non-isomorphic simple $\Lambda$-modules.

Proof. (i) $\Rightarrow$ (ii) By [18, Corollary 12.16], $\Lambda$ is representation finite and has finite global dimension; in particular $\mathcal{H}^{\mathrm{b}}\left(\mathcal{P}_{\Lambda}\right)=\mathbf{D}^{\mathrm{b}}(\bmod -\Lambda)$. Then (ii) follows from Theorem 12.1. The converse follows from Theorem 12.1 and Theorem 9.16 .

Remark 12.4. If $\Lambda$ is a Gorenstein Artin algebra, then from Corollary 12.3 it follows that $\mathbf{D}(\operatorname{Mod}-\Lambda)$ is of finite type iff $\operatorname{art}\left(\mathcal{H}^{\mathrm{b}}\left(\mathcal{P}_{\Lambda}\right)\right)$ is a free basis for the kernel $\Delta\left(\mathcal{H}^{\mathrm{b}}\left(\mathcal{P}_{\Lambda}\right)\right)$ of the Cartan map $\mathrm{K}_{0}\left(\mathcal{H}^{\mathrm{b}}\left(\mathcal{P}_{\Lambda}\right), \oplus\right) \rightarrow \mathrm{K}_{0}\left(\mathbf{D}^{\mathrm{b}}(\bmod -\Lambda)\right)$.

\section{Comments and Conjectures}

Let $\mathcal{C}$ be a compactly generated triangulated category. We have seen in Example 10.5 that $\operatorname{Max}(\mathcal{C}) \subseteq \operatorname{Fin}(\mathcal{C})$ does not necessarily implies that $\operatorname{Fin}(\mathcal{C}) \subseteq \operatorname{Max}(\mathcal{C})$. The counter example was the unbounded derived category 
of a ring with several objects. Clearly this compactly generated triangulated category does not admits a single compact generator. The results of the previous sections suggest the following.

PROBLEM. Let $\mathcal{C}:=\mathbf{D}(\operatorname{Mod}-\Lambda)$ be the unbounded derived category of an associative ring $\Lambda$, or more generally a triangulated category with infinite sums and a compact generator, i.e. $\mathcal{C}$ is monogenic. Assume that $\operatorname{Max}(\mathcal{C}) \subseteq \operatorname{Fin}(\mathcal{C})$. Is it true that $\operatorname{Fin}(\mathcal{C}) \subseteq \operatorname{Max}(\mathcal{C})$ ? If this is not case, then under what conditions is it true?

The problem is related to the following open conjectures in representation theory:

DPSC The Derived Pure Semisimple Conjecture: If $\mathbf{D}(\operatorname{Mod}-\Lambda)$ is puresemisimple, then $\mathbf{D}(\operatorname{Mod}-\Lambda)$ is of finite type.

PSC The Pure Semisimple Conjecture: Any pure-semisimple ring is of finite representation type.

GSC The Gorenstein Symmetry Conjecture: Any Artin algebra $\Lambda$ with $\operatorname{id}_{\Lambda} \Lambda<\infty$, is Gorenstein, i.e. id $\Lambda_{\Lambda}<\infty$.

Note that, by [18, Remark 12.18], DPSC implies PSC and that in the situation of the conjectures DPSC and GSC, the maximal spectrum is contained in the finite spectrum, by Lemma 10.1 and Proposition 9.14 respectively. If the problem has a positive answer, then, by Theorems 9.15 and 10.2, the maximal and the finite spectra coincide in both cases and this implies the validity of the conjectures. Hence the problem can be considered as a generalized form of the three conjectures.

\section{Acknowledgment}

The main part of this work was done during a stay at the University of Bielefeld in 1998 and presented in lectures at Norwegian University of Science and Technology in 1999 and 2002. The author thanks Prof. I. Reiten, Prof. C.M. Ringel and Prof. H. Krause for the warm hospitality and the excellent working conditions. I would like to express my special thanks to Henning Krause for the inspiring discussions and the ideas he contributed in the paper, and the referee for his/her valuable comments and suggestions which resulted in a significant improvement of the article.

\section{References}

1. Assem, I., Beligiannis, A. and Marmaridis, N., Right Triangulated Categories with Right Semi-equivalence, CMS conf. Proc. 24 Amer. Math. Soc. Providence, RI, (1998), pp. 1737.

2. Auslander, M.: Representation theory of artin algebras II, Comm. Algebra 2 (1974), 269-310. 
3. Auslander, M.: Large Modules over Artin algebras, Algebra, Topology, and Category Theory (a collection of papers in honor of Samuel Eilenberg), Academic Press, New York (1976), pp. 1-17.

4. Auslander, M.: Functors and morphisms determined by objects, in R. corden (ed.), Representation Theory of Algebras, Proc. Conf. Philadelphia (1976), Dekker, New York (1978), 1-244.

5. Auslander, M. and Reiten, I.: Representation theory of Artin algebras III. Almost split sequences, Comm. Algebra 3 (1975), 239-294.

6. Auslander, M. and Reiten, I.: Stable Equivalence of dualizing R-varieties, Adv. Math. (1974), 306-366.

7. Auslander, M. and Reiten, I.: Representation theory of Artin algebras IV. Invariants given by almost split sequences, Comm. Algebra 5(5) (1977), 443-518.

8. Auslander, M. and Reiten, I.: Grothendieck groups of algebras and orders, J. Pure Appl. Algebra 39(1-2) (1986), 1-51.

9. Auslander, M. and Reiten, I.: Cohen-Macaulay and Gorenstein Artin algebras., Progr. Math. 95 (1991), 221-245.

10. Auslander, M. and Smalø, S.: Preprojective modules over Artin algebras J. Algebra 66(1) (1980), 61-122.

11. Auslander, M. and Smalø, S.: Almost split sequences in subcategories, J. Algebra 69(2) (1981), 426-454.

12. Auslander, M.: Isolated singularities and existence of almost split sequences, Lect. Notes Math. 1178 (1986), 194-242.

13. Auslander, M., Reiten, I. and Smalø, S.: Representation Theory of Artin Algebras, Cambridge Studies in Advanced Mathematics. 36, Cambridge University Press, Cambridge (1995), xiv $+423 \mathrm{pp}$.

14. Beilinson, A.A., Bernstein, J. and Deligne, P.: Faisceaux pervers, Analysis and topology on singular spaces, I (Luminy, 1981), 5-171, Astérisque, 100, Soc. Math. France, Paris (1982).

15. Beligiannis, A.: On the Freyd Categories of an Additive Category, Homology, Homotopy and Applications, Vol. 2(11) (2000), 147-185.

16. Beligiannis, A.: On the relative homology of cleft extensions of rings and abelian categories, J. Pure Appl. Algebra 150(3) (2000), 237-299.

17. Beligiannis, A.: The homological theory of contravariantly finite subcategories: Auslander-Buchweitz contexts, gorenstein categories and (Co)stabilization, Comm. Algebra 28 (2000), 4547-4596.

18. Beligiannis, A.: Relative homological algebra and purity in triangulated categories, J. Algebra 227 (2000), 268-361.

19. Beligiannis, A.: Purity and almost split morphisms in abstract Homotopy categories: A Unified Approach via Brown Representability, Algebr. Represent. Theory 5 (2002), 483525.

20. Beligiannis, A.: Cohen-Macaulay modules, (Co)Torsion Pairs and Virtually Gorenstein Algebras, preprint, University of the Aegean (2003).

21. Beligiannis, A.: Relative Homology, Tilting objects and Freyd's Generating Hypothesis, preprint in preparation.

22. Beligiannis, A. and Reiten, I.: Homological and Homotopical Aspects of Torsion Theories, preprint (2002).

23. Bondal, A.I. and Kapranov, M.M.: Representable functors, Serre functors, and reconstructions, (Russian) Izv. Akad. Nauk SSSR Ser. Mat. 53(6) (1989), 1183-1205, 1337; Translation in Math. USSR-Izv. 35(3) (1990), 519-541. 
24. Bondal, A.I. and Van den Bergh, M., Generators and representability of functors in commutative and noncommutative geometry, Mosc. Math. J. 3(1) (2003), 1-36.

25. Christensen, J.D.: Ideals in triangulated categories: phantoms, ghosts and skeleta, $A d v$. Math. 136(2) (1998), 284-339.

26. Crawley-Boevey, W.: Modules of Finite Length over their Endomorphism Ring, in S. Brenner and H. Tachikawa (eds.) Representations of Algebras and Related Topics, London Math. Soc. Lec. Note Series 168 (1992), 127-184.

27. Crawley- Boevey, W.: Locally finitely presented additive categories, Comm. Algebra 22 (1994), 1644-1674.

28. Freyd, P.: Stable homotopy, in Proc. Conf. Categorical Algebra, La Jolla (1966), $121-172$.

29. Freyd, P.: Stable homotopy II, Applications of Categorical Algebra, Proc. Sympos. Pure Math., Vol. XVII, New York, (1968), pp. 161-183, Amer. Math. Soc., Providence, R.I.

30. Gabriel, P.: Des Catégories Abéliennes, Bull. Soc. Math. France 90 (1962), 323-448.

31. Gabriel, P. and Roiter, A.V.: Representations of Finite-Dimensional Algebras Translated from the Russian. With a chapter by B. Keller. Reprint of the 1992 English translation. Springer-Verlag, Berlin, 1997. iv +177 pp.

32. Garkusha, G. and Prest, M.: Triangulated Categories and the Ziegler Spectrum, preprint (2002).

33. Geigle, W. and Lenzing, H.: Perpendicular categories with applications to representations and sheaves, J. Algebra 144 (1991), 273-343.

34. Gruson, L. and Jensen, C.U.: Dimensions Cohomologiques Reliées aux Functeurs $\lim ^{(i)}$, in Springer Lecture Notes in Mathematics, vol. 867 (1981), 234-294.

35. Happel, D.: On the Derived Category of a Finite-dimensional Algebra, Comment. Math. Helv. 62 (1987), 339-389.

36. Happel, D.: Triangulated Categories in the Representation Theory of Finite Dimensional Algebras, LMS Lecture Notes 119, Cambridge University Press (1988).

37. Happel, D.: Auslander-Reiten Triangles in Derived Categories of Finite-Dimensional Algebras, Proc. Amer. Math. Soc. 112 (1991), 641-648.

38. Happel, D.: On Gorenstein Algebras, Progress in Mathematics 95 (1991), 389-404.

39. Herzog, I.: The Auslander-Reiten translate, Contemp. Math. 130, Amer. Math. Soc., Providence, RI (1992), 153-165.

40. Herzog, I.: Finitely presented right modules over a left pure-semisimple ring, Bull. London Math. Soc. 26(4) (1994), 333-338.

41. Herzog, I.: The Ziegler spectrum of a locally coherent Grothendieck category, Proc. London Math. Soc. 74(3) (1997), 503-558.

42. Jensen, C.U. and Lenzing, H.: Model-theoretic Algebra with Particular Emphasis on Fields, Rings, Modules, Algebra, Logic and Applications, 2. Gordon and Breach Science Publishers, New York, (1989), xiv +443 pp.

43. Jensen, C.U. and Simson, D.: Purity and generalized chain conditions, J. Pure Appl. Algebra 14 (1979), 297-305.

44. Jørgensen, P.: Auslander-Reiten theory over topological spaces, Comment. Math. Helv. 79 (2004), 160-182.

45. Kaplansky, I.: Infinite Abelian Groups, The University of Michigan Press, Ann Arbor, Mich. (1969), vii + 95 pp.

46. Keller, B. and Vossieck, D.: Sous les catégories dérivées, C. R. Acad. Sci. Paris Sr. I Math. 305(6) (1987), 225-228.

47. Keller, B.: Deriving DG categories, Ann. Sci. cole Norm. Sup. (4) 27(1) (1994), 63-102.

48. Keller, B.: $A_{\infty}$-algebras and triangulated categories, in preparation. 
49. Krause, H.: Exactly definable categories, J. Algebra 201(2) (1998), 456-492.

50. Krause, H.: Decomposing thick subcategories of the stable module category, Math. Ann. 313 (1999), 95-108.

51. Krause, H.: Smashing subcategories and the telescope conjecture-An algebraic approach, Invent. Math. 139 (2000), 99-133.

52. Krause, H.: Auslander-Reiten triangles via brown representability, $K-$ Theory 20 (2000), $331-344$.

53. Krause, H.: The spectrum of a module category, Memoirs A.M.S. 707, (2001).

54. Krause, H.: A Brown representability theorem via coherent functors, Topology 41(4) (2002), 853-861.

55. Krause, H.: A duality between complexes of left and right modules, in D. Happel and Y.B. Zhang (eds.) Representations of Algebras, Vol. I, Proceedings of the Ninth International Conference, Beijing 2000, Beijing Normal University Press (2002), pp. 87-97.

56. Krause, H.: Coherent functors in stable homotopy theory, Fund. Math. 173 (2002), 3356.

57. Krause, H.: A Note on Auslander-Reiten Triangles and a Theorem of Zimmermann, Preprint (2003).

58. Krause, H.: Cohomological Quotients and Smashing Localizations, Preprint (2003).

59. Krause, H. and Reichenbach, U.: Endofiniteness in stable homotopy theory, Trans. Am. Math. Soc. 353(1) (2001), 157-173.

60. Krause, H. and Saorin, M.: On Minimal Approximations of Modules, Contem. Math. 229 (1998), 227-236.

61. Margolis, H.R., Spectra and the Steenrod Algebra, North Holland Math. Library 29 North Holland, (1983).

62. Matlis, E.: Injective modules over Noetherian rings, Pacific J. Math. 8 (1958), 511-528.

63. Neeman, A.: The Brown representability theorem and phantomless triangulated categories, J. Algebra 151(1) (1992), 118-155.

64. Neeman, A.: The Grothendieck duality theorem via Bousfield's techniques and Brown representability, J. Amer. Math. Soc. 9 (1996), 205-236.

65. Neeman, A.: On the derived category of sheaves on a manifold, Doc. Math. 6 (2001), 483488.

66. Neeman, A.: Triangulated Categories, Annals of Mathematics Studies 148, Princeton University Press, vii, 449 p. (2001).

67. Orlov, D.: Triangulated Categories of Singularities and D-Branes in Landau-Ginzburg Models, preprint (2003).

68. Prest, M., Model Theory and Modules, London Mathematical Society Lecture Note Series 130, Cambridge University Press, Cambridge (1988), xviii +380 pp.

69. Prest, M.: Representation embeddings and the Ziegler spectrum, J. Pure Appl. Algebra 113(3) (1996), 315-323.

70. Prest, M.: Epimorphisms of rings, interpretations of modulea and the Ziegler spectrum, Comm. Algebra 24 (1996), 517-531.

71. Prest, M.: The Zariski spectrum of the category of finitely presented modules, preprint (1998).

72. Prest, M.: Topological and Geometric Aspects of the Ziegler Spectrum, Infinite length modules (Bielefeld, 1998), Trends Math., Birkhuser, Basel (2000), pp. 369-392.

73. Quillen, D.: Higher algebraic K-theory I., Lect. Notes Math. 341 (1973), 85-147.

74. Reiten, I.: The use of almost split sequences in the representation theory of Artin algebras, Representations of algebras (Puebla, 1980), Lecture Notes in Math. 944, Springer, BerlinNew York (1982), pp. 29-104. 
75. Reiten, I. and Van den Bergh, M.: Noetherian hereditary abelian categories satisfying Serre duality, J. Amer. Math. Soc. 15(2) (2002), 295-366.

76. Rickard, J.: Morita theory for derived categories, J. London Math. Soc. (2) 39 (1989), 436-456.

77. Verdier, J.-L.: Des catégories dérivées des catégories abéliennes, Astérisque No. 239 (1996), xii + 253 pp. (1997).

78. Wakamatsu, T.: Stable equivalence for self-injective algebras and a generalization of tilting modules, J. Algebra 134 (2)(1990), 298-325.

79. Xiao, J. and Zhu, B.: Relations for the Grothendieck Groups of Triangulated Categories, J. Algebra 257 (2002), 37-50.

80. Ziegler, M.: Model theory of modules, Ann. Pure and Appl. Logic 26 (1984), 149-213.

81. Zimmermann, W.: Auslander-Reiten sequences over Artinian rings, J. Algebra 119(2) (1988), 366-392.

82. Zimmermann-Huisgen, B.: Strong preinjective partitions and representation type of Artinian rings, Proc. Amer. Math. Soc. 109(2) (1990), 309-322.

83. Zimmermann-Huisgen, B. and Zimmermann, W.: On the sparsity of representations of rings of pure global dimension zero, Trans. Amer. Math. Soc. 320(2) (1990), 695-711. 